ISBN 82-553-0817-2

No 20

Pure Mathematics

December 1992

\title{
Brownian Motion on Simple Fractal Spaces
}

by

Svein Olav Nyberg

PREPRINT SERIES - Matematisk institutt, Universitetet i Oslo 
Thanks to Tom Lindstrøm, without whose help this work would not have been possible. This work has been supported by a grant from the Norwegian Research Council, NAVF. 


\section{Contents}

0 Introduction ii

0.1 Fractals and Self-similar spaces .................... ii

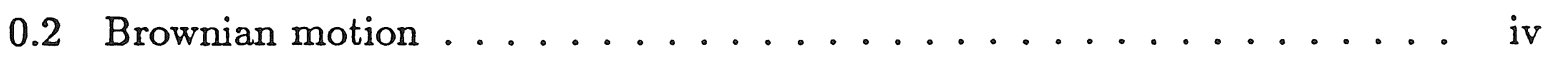

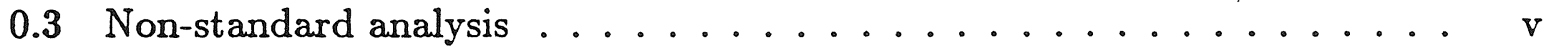

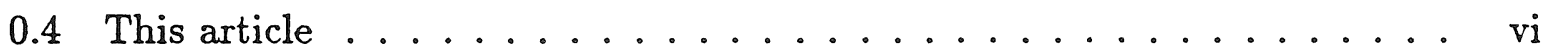

0.5 Simple fractal spaces .............................. vi

0.6 Physical properties and Brownian motion ............ vii

I The Process viii

1 Simple Fractal Spaces 1

1.1 Geometric properties . . . . . . . . . . . . . . . . . 2

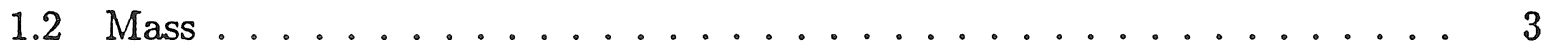

1.3 The electric network on $\mathrm{K} \ldots \ldots \ldots \ldots$

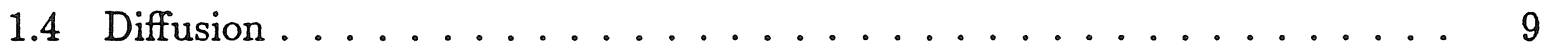

2 Brownian Motion on Simple Fractal Spaces 14

2.1 Some lemmas on $\mathcal{G} \ldots \ldots \ldots \ldots \ldots$

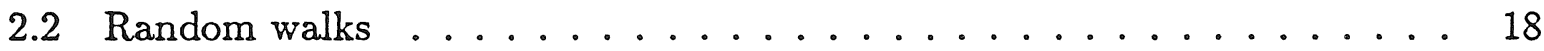

2.3 Decimation Invariant Random walks ............... 25

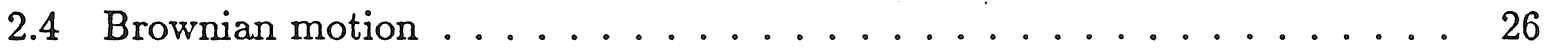


3 The Markov Property $\quad 29$

3.1 Some properties of the process .................... 29

3.2 A random walk with the Markov property . . . . . . . . . . . 30

3.3 The Markov Property ........................... 33

4 Relaxing the Axioms $\quad 36$

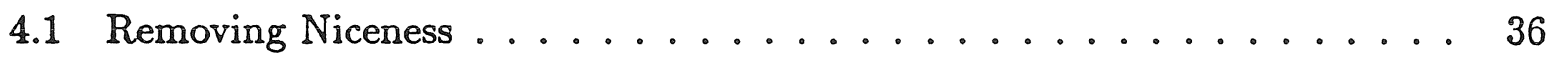

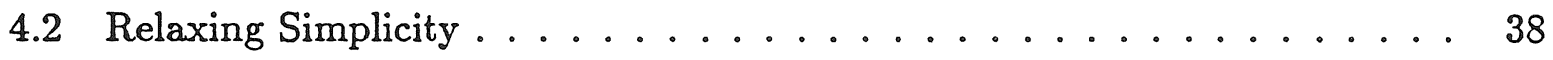

$\begin{array}{lr}\text { II The Space } & 40\end{array}$

5 Simple fractal spaces $\quad 41$

5.1 The topological measure space $A^{\mathbb{N}} \ldots \ldots \ldots . \ldots \ldots$

5.2 The quotient space $\mathrm{K} \ldots \ldots \ldots \ldots \ldots \ldots$

5.3 The topological space $(\mathrm{K}, \Theta) \ldots \ldots \ldots \ldots \ldots$

5.4 The measure space $(\mathbb{K}, \mathcal{B}, \mu) \ldots \ldots \ldots \ldots \ldots$

5.5 The metric space $(\mathrm{K}, \mathrm{d}) \ldots \ldots \ldots \ldots \ldots \ldots$

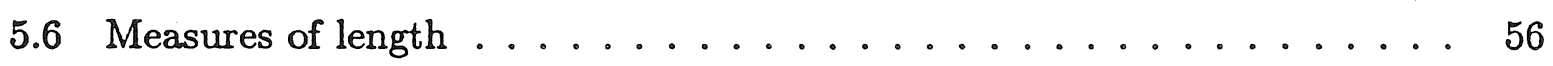

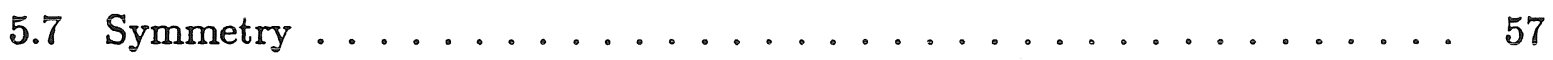




\section{Chapter 0}

\section{Introduction}

Physical processes can be expressed through differential equations. But differential equations presuppose a medium capable of supporting a differentiable structure, which the majority of physical mediums cannot. But physical processes happen all the same. What might first be attempted, is to approximate these structures with differentiable ones. Another approach is to study diffusions on the existing structures, and from them construct the analogs of the differential operators. That is the modern approach.

In mathematics we have known non-differentiable strucures for a long time, the fractals. They have largely had their application as counterexpamples, and have thus acquired the nickname "the monsters of mathematics". Now, they will hopefully serve as a spring board on the journey towards modelling physical processes on non-smooth mediums. Let us have a closer look.

\subsection{Fractals and Self-similar spaces}

Classical Fractals are subspaces of $\mathbf{R}^{n}$ for some natural number $n$. The characteristic of these subspaces, is that their topological dimension, $\operatorname{dim}_{\tau}$, is different from their Hausdorff dimension, $\operatorname{dim}_{\mathcal{H}}$, where the Hausdorff dimension of a set $M$ is defined as

$$
\operatorname{dim}_{\mathcal{H}}(M)=\sup _{r}\left\{\mathcal{H}^{r}(M)=\infty\right\}=\inf _{r}\left\{\mathcal{H}^{r}(M)=0\right\}
$$

where $\mathcal{H}^{r}$ is the $r$-dimensional Hausdorff measure of the set $M$, and is given by

$$
\mathcal{H}^{c}(M)=\lim _{\delta \rightarrow 0} \mathcal{H}_{\delta}^{r}(M)=\lim _{\delta \rightarrow 0}\left(\inf \left\{\sum\left(\operatorname{diam}\left(M_{i}\right)\right)^{r}: M \subset \bigcup_{i \in \mathbb{N}} M_{i},\left(\operatorname{diam}\left(M_{i}\right)\right) \leq \delta\right\}\right)
$$

A Self similar space is a compact topological space $T$, with $T=\bigcup_{i=1}^{k} f_{i}(T)$, where the functions $f_{i}: T \rightarrow T, 1 \leq i \leq k$, are non-surjective, and homeomorphisms upon their image. The most studied among such spaces are the compact subsets of $\mathbb{R}^{n}$, where $f_{i}$ are affine contractions. Most of these spaces are fractals, but also spaces like the interval $[0,1]$ 
are self similar. We give the points of self similar spaces addresses in the following way: If $x=\lim _{n \rightarrow \infty} f_{a_{1}} \circ f_{a_{2}} \cdots \circ f_{a_{n}}(y)$, where $y$ is an arbitrary point (the limit is independent of the starting point), then $\left(a_{1}, a_{2}, \ldots\right)$ is an address of $x$. Given a vector of $n$ symbols, $\vec{a}=\left(a_{1}, \cdots, a_{n}\right)$, the corresponding $n$-cell, $C_{\vec{a}}$, is given by $C_{\vec{a}}=f_{a_{1}} \circ \cdots \circ f_{a_{n}}(T)$.

Let $f=\left\{f_{1}, \ldots, f_{k}\right\}$. We call $f$ an iterated function system. Let $f(M)=\bigcup_{f_{i} \in f} f_{i}(M)$. Then $f$ is a function $\mathcal{P}(T) \rightarrow \mathcal{P}(T)$. If $K$ is compact, $f(K)$ is compact, too.

If $X$ is a metric space, then $\mathcal{H}(X)$, the set of the compact subsets of $X$, is a metric space with a metric defined so that if $K_{1}, K_{2} \in \mathcal{H}(X)$, then

$$
\rho\left(K_{1}, K_{2}\right)=\max \left\{\sup _{x \in K_{1}} \inf _{y \in K_{2}}\{d(x, y)\}, \sup _{x \in K_{2}} \inf _{y \in K_{1}}\{d(x, y)\}\right\}
$$

If $X$ is complete, then $\mathcal{H}(X)$ is complete, and if the $f_{i}$ are contractions on $X$, then $f$ is a contraction on $\mathcal{H}(X)$. In that case, $f$ has a fixed point $F \in \mathcal{H}(X)$.

A generator is an imprecise way of giving $f$. The generators are illustrations that are intended to make the reader get an intuitive 'pictorial' understanding of the fractal and its construction. In illustrations 0.1 and 0.2 , we call b) the generator.

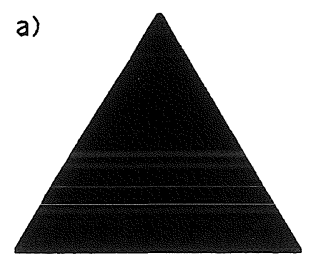

a)

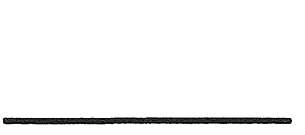

b)

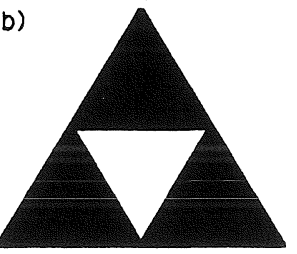

c)

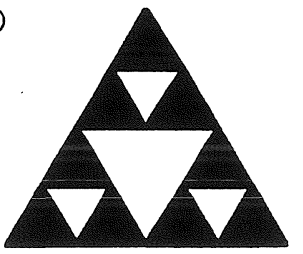

Figur 0.1: Generating the Sierpinski triangle

b)

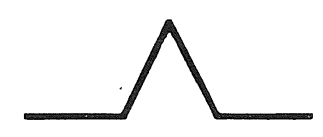

C)

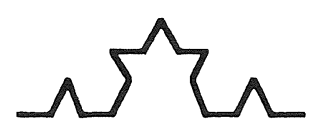

Figur 0.2: Generating the Koch curve

We are to iteratively replace the 'obvious' parts with 'obvious' scaled copies of the whole figure. This will necessarily bring us into some trouble, in that what is 'obvious' is not canonical. Take 0.2 , where the figure might just as well go on like in 0.3 .

Bandt and Stahnke [3] has constructed a metric on self similar sets that staisfy certain criterions, by letting the distance between two points be the minimal $\beta$-dimensional 
a)

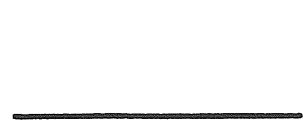

b)

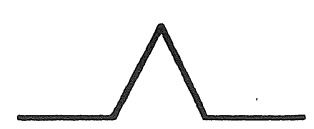

c)

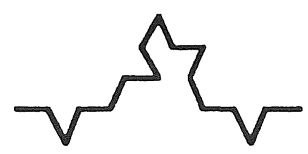

Figur 0.3: Generating the "hcoK curve"

Hausdorff measure of curves connecting the two points. $\beta$ is a constant characteristic of the space. It is this metric that has been the inspiration for the metric in Part II.

\subsection{Brownian motion}

It is a subclass of the self similar spaces that are studied when we study diffusion processes on fractals. Most of these fractals satisfy the axioms set forth by Lindstrøm in his "Brownian Motion on Nested Fractals". An exception is the Sierpinski carpet, which violates "Finite Ramification".

a)

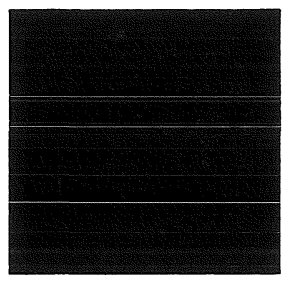

b)

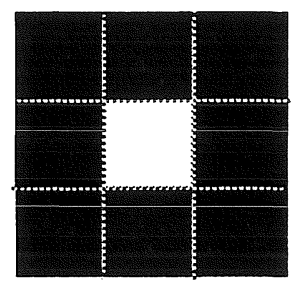

c)

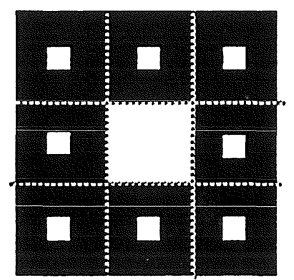

Figur 0.4: Generating the Sierpinski carpet

He starts off with a few conditions on the iterated function system,

(A) $d\left(f_{i}(x), f_{i}(y)\right)=c \cdot d(x, y)$, where $0<c<1$ is a constant independent of $i$. (Guarantees the existence of fixed point)

(B) Open Set Condition There exists an open set $\mathcal{O}$ such that $f(\mathcal{O}) \subset \mathcal{O}$.

and proceeds with the axioms

(i) Connectedness Each pair of 1-cells $C$ and $C^{\prime}$ are connected by a sequence $C_{1}, \ldots, C_{n}$ of 1-cells: $C=C_{1}, C^{\prime}=C_{n}$, and $C_{i} \cap C_{i+1} \neq \emptyset$. 


\section{CHAPTER 0. INTRODUCTION}

(ii) Finite Ramification Given $x_{i}$ as the fixed point of $f_{i}$, define $F^{(0)}$ to be the set of the $x_{i}$ so that there exists a $j \neq i$ and $k, l$ so that $f_{k}\left(x_{i}\right)=f_{l}\left(x_{j}\right)$. Then, given two equally long, but distinct, sequences $\left(a_{1}, \ldots, a_{n}\right),\left(b_{1}, \ldots, b_{n}\right)$ of symbols from $\left\{s: f_{s} \in f\right\}$, we have $f_{a_{1}} \circ \cdots \circ f_{a_{n}}(F) \cap f_{b_{1}} \circ \ldots \circ f_{b_{n}}(F)=f_{a_{1}} \circ \ldots \circ f_{a_{n}}\left(F^{(0)}\right) \cap f_{b_{1}} \circ \cdots \circ f_{b_{n}}\left(F^{(0)}\right)$

(iii) Symmetry If $x$ and $y$ are two points of $F^{(0)}$, and $U_{x, y}$ is reflection in the hyperplane between them, $U_{x, y}$ sends $F^{(0)}$ onto $F^{(0)}$, and $n$-cells onto $n$-cells. If an $n$-cell is intersected by the hyperplane, $U_{x, y}$ maps it onto itself.

Define inductively $F^{(n+1)}=f\left(F^{(n)}\right)$. Then $F^{(n+1)} \supset F^{(n)}$, and $\bigcup_{n \in \mathbb{N}} F^{(n)}$ is dense in $F$.

Lindstrøm constructs random walks $B_{n}$ on på $F^{(n)}$, so that the random walk $B_{n+N}$ induces on $F^{(n)}$ is $B_{n}$. It is not too difficult to see that we can find a limit of these random walks, and that this limit is the standard part of $B_{\mathbf{N}}$ for for some $N \in^{*} \mathbf{N}-\mathbf{N}$.

\subsection{Non-standard analysis}

There exists non-standard models for the natural numbers, ${ }^{*} N$, in which the standard model, $\mathbf{N}$, can be embedded. That is, there exists an element $N \in^{*} \mathbf{N}$ such that $N>n$ for any standard natural number $n \in \mathbf{N}$. Such a number is called infinite. Taking mulitplicative inverses, $0<\frac{1}{N}<\frac{1}{n}, \forall n \in N$. We say a number $x$ is near $y$ if $|x-y|<$ $\frac{1}{n}, \forall n \in \mathbf{N}$, and write $x \simeq y$. If $y$ is standard, i.e. $y \in \mathbf{R}$, and $x$ is non-standard, i.e. $x \in^{*} \mathbf{R}$, we say $y$ is the standard part of $x$, and write $y={ }^{\circ}(x)$. If ${ }^{\circ}(x)=0$, we say $x$ is infinitesimal.

Non-standard analysis is very useful when we are taking limits. If $a_{n}$ converges to $a$, $\left\{a_{n}\right\}_{n \in \mathbb{N}}$ is Cauchy,

$$
\forall \varepsilon \in \mathbf{R}^{+} \exists n \in \mathbf{N} \forall m, n \in \mathbb{N}\left(m>N \wedge n>N \rightarrow\left|a_{n}-a_{m}\right|<\varepsilon\right)
$$

We can extend this standard sequence $\left\{a_{n}\right\}_{n \in \mathbb{N}}$ to a non-standard sequence $\left\{a_{n}\right\}_{N \epsilon^{*} \mathbb{N}}$ by a method called $*$-transfer, which is a mapping from the standard to the non-standard model characterized by that it preserves all properties described by first-order sentences. The *-transfer of the sentence above is

$$
\forall \varepsilon \in^{*} \mathbf{R}^{+} \exists N \in^{*} \mathbf{N} \forall m, N \in^{*} \mathbf{N}\left(m>N \wedge n>N \rightarrow\left|a_{n}-a_{m}\right|<\varepsilon\right)
$$

So, if $M, N \in \in^{*} \mathrm{~N},\left|a_{N}-a_{M}\right|$ is infinitesimal, and the standard limit of the standard sequence can be found as $\lim _{n \rightarrow \infty} a_{n}={ }^{\circ}\left(a_{N}\right)$ where $N$ is any infinite number.

These are the basic ideas of non-standard analysis. More extensive treatment can be found in [7], [10] or [1]. 


\subsection{This article}

An interesting question concerning Brownian motion on fractals is what happens when the constant $c$ in (A) is not independent of $i$, that is - the 1-cells are allowed to be of different sizes. What happens, for instance, with transition probabilities? The answering of this question is the main focus of this paper..

To limit the complexity that comes from a random walk over a cell having multiple end stations, I have limited the main study to the fractals where $F^{(0)}$ has 2 elements. Do see, however, the discussion in chapter 4 of the removal of this restriction.

During the search for the transition probabilities I found that the most useful test examples were fractals that werent embeddable in any Euclidean space - virtual fractals, like, for instance, those we would get from the virtual generators

(a) $([0,1] \times\{a, b\}) / \sim$, where $(x, a) \sim(x, b)$ hvis $x \notin\left(\frac{1}{3}, \frac{2}{3}\right)$

(b) $([0,1] \times\{a, b\}) / \sim$, where $(x, a) \sim(x, b)$ hvis $x \in\left[\frac{1}{3}, \frac{2}{3}\right] \cup\{0,1\}$

a)

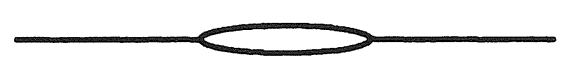

b)

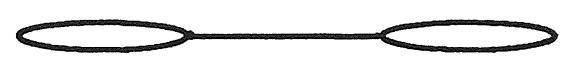

Figur 0.5: Suggestive illustration of virtual generators

\subsection{Simple fractal spaces}

The simple fractal spaces will simply be the virtual fractals that have connected, symmetric generators, and across which the shortest route between the two points in $F^{(0)}$ is of length 1. They will not be constructed as subspaces of any other spaces. This has the advantage that the ambiguity of the generators is removed. This ambiguity would remain if we stuck to embeddings in $\mathbb{R}^{n}$.

Though the spaces have intrinsic interest, my aim is their use in the later study of physical phenomena, in particular Brownian motion. I have therefore put emphasis on making the axioms both in Part I and in Part II as simple and as easily checked as possible. The axioms in Part II imply the axioms in Part I, except for the axiom of Niceness.

I have made a few generators whose fractal will be good to keep in mind while reading Part II. It will give some understanding of why what has been done has been done. 


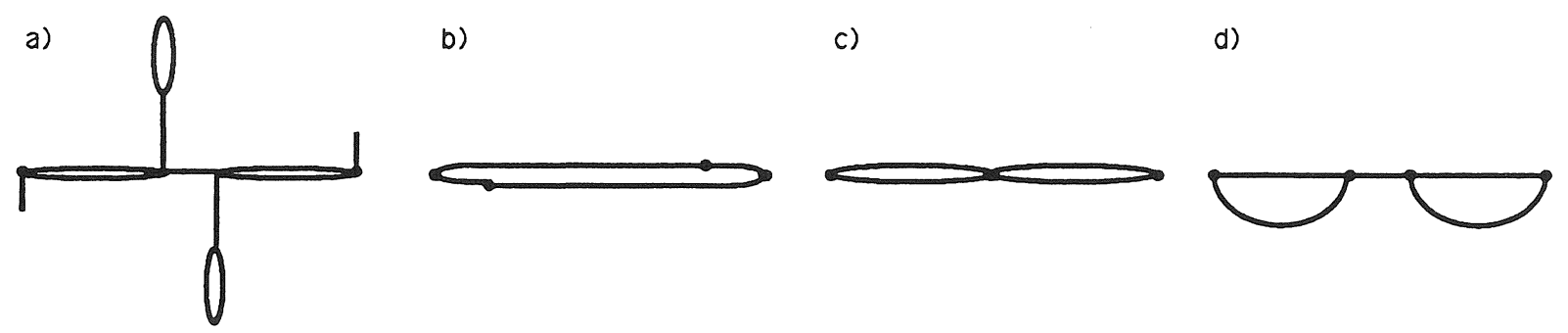

where d) is the generator $X$ given by

$$
X=\{a\} \times[0,1] \cup\{b\} \times\left[0, \frac{5}{3}\right] / \sim
$$

where $(a, 0) \sim(b, 0),(a, 1) \sim\left(b, \frac{5}{3}\right)$

and $(a, x) \sim(b, y)$ if $x \in\left[\frac{1}{3}, \frac{2}{3}\right]$ and $y=x+\frac{1}{3}$.

\subsection{Physical properties and Brownian motion}

The spaces on which the main work is done, in Part I, are the Simple Fractal spaces with the Niceness property, i.e. that the end points of the fractal are contained in one 1-cell only. That excludes the fractals given in 0.6. The challenge is to expand the theory to fit them in. See, however, chapter 4.

The general idea is that I first construct some random walks $X^{(n)}$ on $F^{(n)}$, and then define the Brownian motion as $\lim _{n \rightarrow \infty} X^{(n)}$. This is, however, not as easy as it seems, since $\left.X^{(n)}\right|_{F(m)},(m<n)$ in general is not equal to $X^{(m)}$. What I do, roughly, is that I define $W_{n}^{(m)}=\left.X^{(n)}\right|_{F(m)}$, and let $W^{(m)}=\lim _{n \rightarrow \infty} W^{(n)} \simeq^{0}\left(W_{N}^{(m)}\right)$ for $N \in^{*} N-N$. Then, $\left.\left.W^{(n)}\right|_{F^{(m)}} \simeq W_{N}^{(n)}\right|_{F^{(m)}}=W_{N}^{(m)} \simeq W^{(m)}$, and we have $\left.W^{(n)}\right|_{F^{(m)}}=W^{(m)}$. This property is called decimation invariance. The Brownian motion is then the limit as $n$ goes to infinity of $W^{(n)}$, or the standard part of $W^{(N)}$ for $N \in^{*} N-N$.

Now, there are two properties of the random walk that we need to keep track of to make our walks. They are transition probability, and transition time. Since it is quite unreasonable to expect a Brownian motion to traverse two cells whose sizes differ, equally fast, or with equal probability, we need to find what would be reasonable to expect in such cases. It turns out that the answer lies in what I will call geometric properties of scaling.

To make transition probabilities decimation invariant, I have used the theory of random walks modelled by electrical networks. The problem now becomes the easier one of finding decimation invariant electrical networks on the $F^{(n)}$. 


\section{Part I}

\section{The Process}




\section{Chapter 1}

\section{Simple Fractal Spaces}

A Simple Fractal space with the Niceness property is a symmetric p.c.f fractal with two fixed points as ramification points. The space is thus a self-sufficient metric space, requiring no embedding in any other metric space (e.g. $\mathbf{R}^{n}$ ), and differs from fractals where Brownian Motion has been previously studied in that these spaces allow for the cells to be of different sizes.

Put more concretely, a Simple Fractal Space with the Niceness property (SN space for short) is a complete metric space with set of injective maps indexed by a finite set, $\left(\mathbf{K}, d,\left\{\phi_{a}\right\}_{a \in A}\right)$, satisfying:

Axiom 1 [Self similarity]

$$
\forall a \in A \exists \varepsilon>0, r \in(0,1) \forall x, y \in \mathbf{K}\left(d(x, y) \leq \varepsilon \rightarrow d\left(\phi_{a}(x), \phi_{a}(y)\right)=r \cdot d(x, y)\right)
$$

The unique $r$ belonging to $\phi_{a}$ will be denoted $r_{a}$. We will let $r_{\min _{1}}=\min \left\{r_{a} \mid a \in A\right\}$ and $r_{\max _{1}}=\max \left\{r_{a} \mid a \in A\right\}$. We can further compose maps, so that if $\vec{a}=\left(a_{1}, \ldots, a_{n}\right) \in A^{n}$, then $\phi_{\vec{a}}=\phi_{a_{1}} \circ \cdots \circ \phi_{a_{n}}$, and $\phi_{\vec{a}}$ has an $r$ belonging to it, that we will call $r_{\vec{a}}$. We immediately get $r_{\vec{a}}=r_{a_{1}} \cdots r_{a_{n}}$. Letting $C_{\vec{a}}=\phi_{\vec{a}}(\mathbf{K})$, with $\vec{a} \in A^{n}$, we call $C_{\vec{a}}$ an $n$-cell. $\phi_{\emptyset}$ will by convention be the identity map on $\mathbf{K}$, and $C_{\emptyset}=\mathbf{K}$.

Axiom 2 [Self similarity 2] $\mathbf{K}$ is the unique fixed point of the map $\psi: \mathcal{H}(\mathbf{K}) \rightarrow \mathcal{H}(\mathbf{K})$ defined by $\psi(B)=\bigcup_{a \in A} \phi(B)$.

$\mathcal{H}(\mathbf{K})$ is the space of all non-empty compact subsets of $\mathbf{K}$ endowed with the Hausdorff metric; for further discussion on $\mathcal{H}(X)$, see $[5,15]$.

Axiom 3 [Simplicity] The set $F^{(0)}=\bigcup_{i \in A} \bigcup_{\substack{j \in A \\ j \neq i}} \phi_{i}^{-1}\left(C_{i} \cap C_{j}\right)$ has cardinality 2, and the elements of $F^{(0)}$ are fixed points for some $\phi_{i}, \phi_{j}, i, j \in A$.

We denote $F^{(0)}$ 's elements $\underline{0}$ and $\underline{1}$. For $n \geq 1$, we define $F^{(n)}=\bigcup_{\vec{a} \in A^{n}} \phi_{\vec{a}}\left(F^{(0)}\right)$. Also, letting $A^{*}=\bigcup_{n \in N_{0}} A^{n}$, we define $F^{(\infty)}=\bigcup_{\vec{a} \in A^{*}} \phi_{\vec{a}}\left(F^{(0)}\right)$ 
Axiom 4 [Connectedness] $(\mathbf{K}, d)$ is connected.

Axiom 5 [Symmetry] There is a self-inverse isometry $U: \mathbb{K} \rightarrow \mathbf{K}$ such that $U(\underline{0})=1$ and $n$-cells are mapped onto $n$-cells for all $n \in \mathbb{N}$.

Axiom 6 [Niceness] $\forall x \in F^{(0)} \exists ! a \in A\left(x \in \phi_{a}(\mathbf{K})\right)$

We shall, and without loss of generality, include what is called the 4th metric axiom

Axiom $7 \forall x, y \in \mathbb{K} \exists z \in \mathbf{K}(d(x, y)=d(x, z)+d(z, y))$

We see that this does not give away generality, by using Bandt's construction of the "internal metric" [3] $d^{\prime}$ given by $d$ :

$$
d^{\prime}(x, y)=\min \left\{\mathcal{H}^{\beta}(S) \mid S \text { is connected and } x, y \in S\right\}
$$

where $\beta$ is an exponent characteristic to $(\mathbf{K}, d)$.

What happens when we make this "internal metric", is that we for each real number $\beta^{\prime} \geq 1$ look at the $\beta^{\prime}$ dimensional Hausdorff measure of the different paths between two points $x, y \in \mathbf{K}$, and take infimum over these measures. Let this infimum be $i\left(\beta^{\prime}\right)$. Then, we let $\beta_{x y}$ be the number where $i$ jumps from infinity to zero. Bandt has shown that for fractals satisfying certain regularity conditions, $\beta_{x y}$ is independent of $x$ and $y . \beta$ is this common number.

That this restriction happens without loss of generality, depends on the observation the reader will make as he reads, that all necessary information is coded into the $r_{a}$ from Axiom 1, and is brought out only in the form of exponents of these numbers. Seeing that the change from the initial metric to the internal metric gives us just a new $r_{a}^{\prime}=r_{a}^{\frac{1}{\beta}}$, we see that nothing is lost. So we can say we are studying equivalence classes of simple fractal spaces, where $\left(\mathbf{K}, d_{1},\left\{\phi_{a}\right\}_{a \in A}\right) \sim\left(\mathbf{K}, d_{2},\left\{\phi_{a}\right\}_{a \in A}\right)$ whenever $d_{1}^{\prime}=d_{2}^{\prime}$.

\subsection{Geometric properties}

I attempt to answer the question "What is the "natural" diffusion on a given fractal?" by answering that it is one which is given by the geometry in some obvious way. In particular, all aspects of it should scale right when we map from the whole of the fractal down to one cell. The scaling should be geometric, in the sense that if $p$ is a property of the diffusion which can be expressed as a function of the cells of $\mathbf{K}$, so that $p$ is a function from $A^{*} \rightarrow \mathbf{R}_{0}^{+}$, then it satisfies the following definition:

Definition 1 A function $p: A^{*} \rightarrow \mathbf{R}_{0}^{+}$is geometric if

1. $p$ is monotone with $r_{\vec{a}}$.

2. $p(\vec{a} \oplus \vec{b})=p(\vec{a}) \cdot p(\vec{b})$, 
where $\left(a_{1}, \ldots, a_{n}\right) \oplus\left(b_{1}, \ldots, b_{m}\right)=\left(a_{1}, \ldots, a_{n}, b_{1}, \ldots, b_{m}\right)$.

Lemma 1.1.1 If $p: A^{*} \rightarrow \mathbb{R}_{0}^{+}$is geometric, then there exists a $q \in \mathbb{R}$ such that $p(\vec{a})=$ $\left(r_{\vec{a}}\right)^{q}$.

Proof: For each $i \in A$ we can find a $q_{i}$ such that $\left(r_{i}\right)^{q_{i}}=p(i)$. We want to show that $q_{i}$ has to be the same for all $i \in A$. Observe that $p\left(a_{1}, \ldots, a_{n}\right)=\prod\left(r_{a_{i}}\right)^{q_{a_{i}}}$.

We need only regard the case where we have $i, j \in A$ with $r_{i} \neq r_{j}$, and the rest will follow by induction.

Note that if $r_{i}=r_{j}^{\frac{t}{s}}$ for any $s, t \in \mathrm{N}$, then $\left(r_{i}^{s}\right)^{q_{i}}=\left(r_{j}^{t}\right)^{q_{j}}$, which implies $q_{i}=q_{j}$.

Applying non-standard analysis, we see there exists $s, t \in^{*} N-N$ such that $r_{j}^{\frac{t}{s}} \leq r_{i} \leq$ $r_{j}^{\frac{t+1}{s}} \simeq r_{j}^{\frac{t}{s}}$.

Since (1) requires the sign of $q_{i}$ and $q_{j}$ to be the same, we can without loss of generality assume them positive. [ Otherwise, regard $p^{-1}$.]

Then $\left(r_{j}^{q_{j}}\right)^{\frac{t}{s}} \leq r_{i}^{q_{i}} \leq\left(r_{j}^{q_{j}}\right)^{\frac{t+1}{s}} \simeq\left(r_{j}^{q_{j}}\right)^{\frac{t}{s}}$, by (1). But then we have both

$$
\begin{array}{r}
r_{j} \leq\left(r_{i}^{\frac{s}{t}}\right)^{\frac{q_{i}}{q_{j}}} \leq r_{j} \cdot r_{j}^{\frac{1}{s}} \simeq r_{j} \\
r_{j} \leq r_{i}^{\frac{s}{t}} \leq r_{j} \cdot r_{j}^{\frac{1}{s}} \simeq r_{j} \text { and }
\end{array}
$$

so $r_{i}^{\frac{s}{t}} \simeq\left(r_{i}^{\frac{s}{t}}\right)^{\frac{q_{i}}{q_{j}}}$, making $q_{i} \simeq q_{j}$. But since both are real, they are equal.

\subsection{Mass}

The first application of the criterion of geometricity is to the mass measure on the fractal. If $\mu$ is a measure on $\mathbf{K}$, it will be called a geometric measure if the function $w: A^{*} \rightarrow \mathbb{R}_{0}^{+}$ defined by $w_{\vec{a}}=\mu\left(C_{\vec{a}}\right)$ is geometric.

Lemma 1.2.1 For $\mu$ and $w$ as given above, there exists a uniquely given $D$ such that

$$
w_{\vec{a}}=\left(r_{\vec{a}}\right)^{D} \forall \vec{a} \in A^{*} \text { and } \sum_{i \in A} w_{i}=\sum_{i \in A}\left(r_{i}\right)^{D}=1
$$

Proof: Existence: $w$ is geometric, so applying lemma 1.1 .1 to it, we have $\mu\left(C_{\vec{a}}\right)=$ $w_{\vec{a}}=\left(r_{\vec{a}}\right)^{d}$ for a real number $d$. 
Uniqueness: It is easily seen (see also 5.4.1), that if $x \in F^{(n)}$, then $\mu(x)=0$. Thus $\mu\left(F^{(\infty)}\right)=0$, and so $\mu(\mathbf{K})=\sum_{a \in A} \mu\left(C_{a}\right)$. Let therefore $f(x)=\sum_{i \in A}\left(r_{i}\right)^{x}$. Then $f$ is a continuous and monotone function with $f(\boldsymbol{R})=\mathbf{R}^{+}$. By monotonicity $f$ assumes every value exactly once. $D=f^{-1}(1)$ is the number we are looking for.

We call $D$ the self similarity dimension of $\mathbf{K}$.

Proposition 1.2.2 $D$ is also the Hausdorff dimension of $\mathbf{K}$, and $0<\mathcal{H}^{D}(K)<\infty$.

Proof: We first prove $D \geq \operatorname{dim}_{H} \mathbf{K}$ and $\mathcal{H}^{D}(\mathbf{K})<\infty$ :

$$
\begin{aligned}
\mathcal{H}_{\delta}^{D}(\mathbf{K}) & =\inf \left\{\sum_{i=1}^{\infty}\left|U_{i}\right|^{D}: \bigcup_{i=1}^{\infty} U_{i} \supseteq \mathbf{K},\left|U_{i}\right| \leq \delta\right\} \\
& \leq \sum_{\vec{a} \in A^{n}}\left(\operatorname{diam}\left(C_{\vec{a}}\right)\right)^{D}\left(\text { with } n \text { so small that } \operatorname{diam}(\mathbf{K}) \cdot r_{\max _{n}} \leq \delta\right) \\
& =(\operatorname{diam}(\mathbf{K}))^{D}
\end{aligned}
$$

for each $\delta>0$, so $\mathcal{H}^{D}(\mathbf{K}) \leq(\operatorname{diam}(\mathbf{K}))^{D}$.

Then we prove $D \leq \operatorname{dim}_{H} \mathbf{K}$ and $\mathcal{H}^{D}(\mathbf{K})>0$ :

Because of Axiom 6, there is a number $M$ such that for all points $x \in \mathbf{K}$, at most $M$ cells can contain $x$, when no cell contains another. For a cell, $C_{\vec{a}}, r_{\min _{1}} \cdot r_{\vec{a}} \leq \operatorname{diam}\left(C_{\vec{a}}\right) \leq$ $\operatorname{diam}(\mathbf{K}) \cdot r_{\vec{a}}$, so the contraction factor of a cell $C_{\vec{a}}, r_{\vec{a}}$, is at most $r_{\min _{1}}^{-1} \cdot \operatorname{diam}\left(C_{\vec{a}}\right)$.

So if $B_{\delta}(x)$ is a ball with center $x$ and radius $\delta$, it can be covered by cells not containing each other, of diameter between $\delta$ and $r_{\min _{1}}^{-1} \cdot \delta$. The maximum number of cells needed for this is $2 M-1$. Since the mass of the ball is less than or equal to the sum of the masses of the cells containing it,

$$
\mu\left(B_{\delta}(x)\right) \leq(2 M-1)\left(r_{\min _{1}}^{-2} \cdot \delta\right)^{D}
$$

But any set $U$ of diameter $\delta$ can be covered by a ball of radius $\delta$, so its mass is not greater than the mass of such a ball:

$$
\mu(U) \leq\left[(2 M-1) r_{\min _{1}}^{-2 D}\right] \cdot|U|^{D}
$$

By the mass distribution principle $\left[9\right.$, Theorem 4.2], $\mathcal{H}^{D}(\mathrm{~K}) \geq \frac{1}{(2 M-1) r_{\min _{1}}^{-2 D}}$, and so $D \leq \operatorname{dim}_{H} \mathbf{K}$.

This is in essence Hutchinson's theorem [11, Theorem 5.3.1] adapted to nice fractal spaces. 


\subsection{The electric network on $\mathrm{K}$}

The study of a medium as an electrical network is interesting beacuse it tells us something about the conductance of the medium in general. In Doyle \& Snell [8] we can see that, the transition probabilities for reversible random walks on a net can be found by considering the associated electrical network and the relation between the current outputs of the different end points, given a potential $v_{s}$ on the starting point and potential $v_{e}$ uniformly on the end points. By finding the geometric resistance, one can thereby find the transition probabilities for the random walks induced by the geometric diffusion.

A resistance ${ }^{1}$ depends on 3 factors: The two points between which the resistance is measured, and the mediating meduim over which current is allowed to go. Take, for instance, the following circuit:

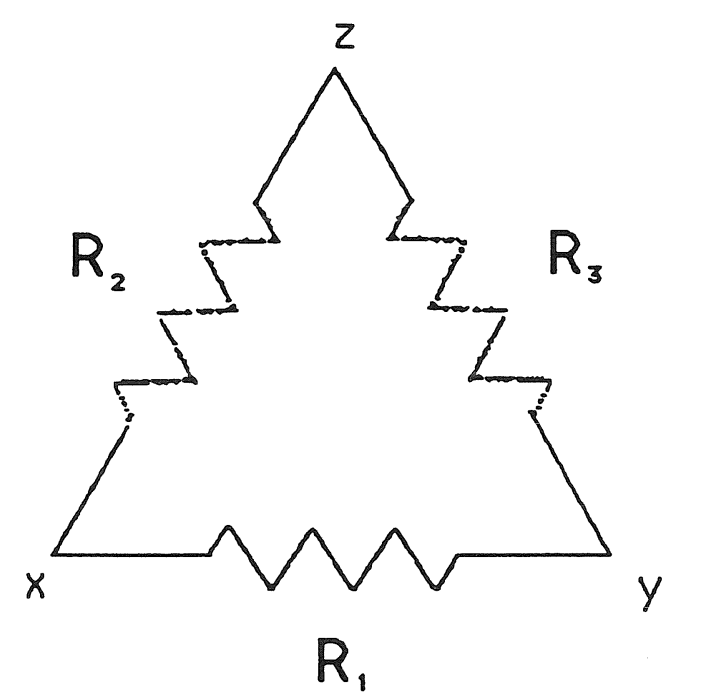

The resistance between $x$ and $y$ given $R_{1}$ as the mediating medium, is simply $R_{1}$. But, if we take the whole circuit as our medium, the resistance is $\frac{1}{\frac{1}{R_{1}}+\frac{1}{R_{2}+R_{3}}}$.

So, given a space $\mathbf{X}$, a resistance function on it, $\rho$, will be written $\rho(x, y, S)$, where $x, y \in \mathbf{X}$ are the points between which the resistance is measured, and $S \subset \mathbf{X}$ is the medium over which we measure it.

We cannot always define $\rho$ so that it is finite for all points in $\mathbf{X} \times \mathbf{X}$. The solution is either, like in $\mathbf{R}^{3}$, to loosen the demand that $\rho$ measures resistance between points, to that it measures resistance between sets of positive measure, for some appropriate measure.

\footnotetext{
${ }^{1}$ We are talking about simple resistance, i.e. the inverse of the current between two points when a unit voltage difference is applied to the same two points. The effective resistance for a given situation can be found once the simple resistance is given.
} 


\section{CHAPTER 1. SIMPLE FRACTAL SPACES}

Or, as will be the most useful in our context, restrict $\rho$ to measure resistance between points in some [dense] subset $\mathcal{Q}$ of $\mathbf{X}$.

Also, we must be careful which sets we allow the resistance to be measured over. Take the unit sphere in $\mathbf{R}^{3}, S^{2}$ to be our space $\mathbf{X}$. Let $x$ and $y$ be the poles, and let the sets $S_{M}$ be defined by

$$
S_{M}=\{(\theta, \phi) \mid \theta \in M\}
$$

The inverse of the resistance, the conductance, then becomes a measure on $S^{1}$, i.e.

$$
\mu(M)=\frac{1}{\rho\left(x, y, S_{M}\right)}
$$

If the resistance is rotation-invariant, $\mu$ will be Lesbegue measure on $S^{1}$. As it is known that not all subsets of $S^{1}$ are Lesbegue-measurable, $\mu$ must be restricted to only a subset of $\mathcal{P}\left(S^{1}\right)$. That, again, implies that $\rho$ must be restricted too. The implication for the general case is that we must restrict the domain of $\rho$ s 3rd argument to a subset $\mathcal{C}$ of $\mathcal{P}(\mathbf{X})$. So, $\rho$ will usually be a function: $\{(x, y, S) \in \mathcal{Q} \times \mathcal{Q} \times \mathcal{C} \mid x, y \in S\} \rightarrow \mathbb{R}_{0}^{+}$. I will let $\mathcal{R}=\{(x, y, S) \in \mathcal{Q} \times \mathcal{Q} \times \mathcal{C} \mid x, y \in S\}$

Now, let us return to our fractals. We will be interested in the $\rho$ where $\mathcal{Q}=F^{(\infty)}$ and $\mathcal{C}$ is the algebra generated by $\left\{C_{\vec{a}} \mid \vec{a} \in A^{*}\right\}$.

Definition 2 For $\rho$ to be a resistance on $\mathcal{R}$, we will have to have certain consistency criterions satisfied, so that composite resistances will not be different from simple resistances.

1. If $C_{\vec{a}} \in \mathcal{C}$ is an $n$-cell, then for any $m \geq n$, the composite resistance over the electrical network $\left\{C_{\vec{b}} \mid \vec{b} \in A^{m} \wedge C_{\vec{b}} \subset C_{\vec{a}}\right\}$, where each cell $C_{\vec{b}}$ is a simple resistance with resistance $\rho\left(\phi_{\vec{b}}(x), \phi_{\vec{b}}(y), C_{\vec{b}}\right)$, equals $\rho\left(\phi_{\vec{a}}(x), \phi_{\vec{a}}(y), C_{\vec{a}}\right)$.

2. If $S \in \mathcal{S}$ is not a cell, let $\eta=\min \left\{n \mid x, y \in F^{(\eta)} \wedge \exists\left\{\vec{a}_{i}\right\}_{i \in \mathcal{I}} \in A^{\eta}\left(\mathcal{S}=\bigcup_{i \in \mathcal{I}} C_{\vec{a}_{i}}\right)\right\}$. $\rho(x, y, S)$ is equal to the composite resistance over the electrical network of $\eta$-cells $\left\{C_{\vec{b}} \mid \vec{b} \in A^{\eta} \wedge C_{\vec{b}} \subset S\right\}$.

3. $\rho(x, y, S)=0 \Rightarrow x=y$.

Criterion 2 is merely what is required to expand a resistance function defined on $\left\{C_{\vec{a}} \mid \vec{a} \in\right.$ $\left.A^{*}\right\}$ only, to one defined on all of $\mathcal{C}$.

Definition $3 \rho: \mathcal{R} \rightarrow \mathbf{R}_{0}^{+}$is a geometric resistance when

1. For all $\vec{a} \in A^{*}$ we can define a $\rho_{\vec{a}}$ such that

$$
\rho\left(\phi_{\vec{a}}(x), \phi_{\vec{a}}(y), \phi_{\vec{a}}(S)\right)=\rho_{\vec{a}} \cdot \rho(x, y, S) \quad \forall(x, y S) \in \mathcal{R}
$$


2. $\rho_{\vec{a}}$ is geometric.

3. $\rho$ is a resistance.

Proposition 1.3.1 Given $\rho$, a geometric resistance, there is a unique constant $R$ such that $\rho_{\vec{a}}=\left(r_{\vec{a}}\right)^{R}$.

Proof: Existence: By 1.1.1 we see that there must be some constant $p$ such that $\rho_{\vec{a}}=\left(r_{\vec{a}}\right)^{p}$.

Uniqueness: We will here make use of condition 1. of definition 2. We need only show that $\rho(\underline{0}, \underline{1}, \mathbf{K})$ equals the composite resistance of the electrical network $\left\{C_{a} \mid a \in A\right\}$ for only one exponent, $R$, i.e. that $\rho_{\vec{a}}=\left(r_{\vec{a}}\right)^{R}$. By definition 3's 1st condition, we then get that for all $n \in \mathbb{N}$ we get that for all $\vec{a} \in A^{n}, \rho\left(\phi_{\vec{a}}(\underline{0}), \phi_{\vec{a}}(\underline{1}), C_{\vec{a}}\right)$ equals the composite resistance of the electrical network $\left\{C_{\vec{a} \oplus b} \mid b \in A\right\}$. This again implies that for any $m \in \mathbb{N}, \rho\left(\phi_{\vec{a}}(\underline{0}), \phi_{\vec{a}}(\underline{1}), C_{\vec{a}}\right)$ equals the composite resistance of the electrical network $\left\{C_{\vec{a} \oplus \vec{b}} \mid \vec{b} \in A^{m}\right\}$

Given a $p \in \mathbf{R}$ we can find a positive $k$ so that the resistance over $C_{i}, \rho_{i}(p)=k \cdot\left(r_{i}\right)^{p}$ uniformly. Let $f(p)$ be the composite resistance, given $p$ as the exponent. With $\varepsilon>0$ fixed, we see that $\frac{\rho_{i}(p+\varepsilon)}{\rho_{i}(p)}=r_{i}^{\varepsilon}$, and $r_{\min _{1}}^{\varepsilon} \leq \frac{\rho_{i}(p+\varepsilon)}{\rho_{i}(p)} \leq r_{\max _{1}}^{\varepsilon}$, so that $r_{\min _{1}}^{\varepsilon} \leq \frac{f(p+\varepsilon)}{f(p)} \leq r_{\max _{1}}^{\varepsilon}$. This means that $f$ is continuous, one-to-one, and that $f(\mathbb{R})=\mathbb{R}^{+}$. Thus, we can find a unique $R \in \mathbb{R}$ such that $f(R)=1$.

We call $R$ the resistance exponent of $\mathbf{K}$.

Now, we have shown the uniqueness of $\rho$, if there indeed exists such a function. Existence needs to be proved.

Corollary 1.3.2 There exists a geometric resistance $\rho: \mathcal{R} \rightarrow \mathbb{R}_{0}^{+}$.

Proof: We can find $R$ as in the uniqueness proof of the previous lemma. Then we have a function $\rho\left(\phi_{\vec{a}}(\underline{0}), \phi_{\vec{a}}(\underline{1}), C_{\vec{a}}\right)$ defined for all $\vec{a} \in A^{*}$.

We expand the function to all of $\mathcal{R}$ by using criterion 2 from definition 2 as a constructive rule.

Lemma 1.3.3 $R>0$.

Proof: Let $\underline{0} \in C_{a}$. Then $1=\rho(\underline{0}, \underline{1}, \mathbf{K})>\rho\left(\phi_{\vec{a}}(\underline{0}), \phi_{\vec{a}}(\underline{1}), C_{\vec{a}}\right)\left(r_{a}\right)^{R}$. 
Lemma 1.3.4 There exists a $k \in(0, \infty)$ such that for any two points $x, y \in F^{(\infty)}$, $\rho(x, y, \mathbf{K}) \leq k$

Proof: Since $\rho(x, z) \leq \rho(x, y)+\rho(y, z)$, we need to show that $\rho(x, \underline{0})$ is finite for all $x \in F^{(\infty)}$. If $x \in F^{(1)}$, then $\rho(x, \underline{0}) \leq \sum_{a \in A}\left(r_{a}\right)^{R}$. Now, if $x \in F^{(n+1)}$, let $C_{\vec{a}}$ be the $n$-cell $x$ is contained in. Then there is a point $y \in F^{(n)} \cap C_{\vec{a}}$. By the geometricity of $\rho$, we now get $\rho(x, y) \leq\left(r_{\vec{a}}\right)^{R} \cdot \sum_{a \in A}\left(r_{a}\right)^{R}$. Since $R>0$, we now can find a chain of elements $x_{n+1}, x_{n}, \ldots, x_{1}, x_{0}$, with $x_{i} \in F^{(i)}, x=x_{n+1}$ and $x_{0}=\underline{0}$, where $\rho\left(x_{i}, x_{i-1}\right) \leq\left(r_{\max _{1}}\right)^{i R} \cdot \sum_{a \in A}\left(r_{a}\right)^{R}$, we finally get, using (3) repeatedly, that $\rho(x, \underline{0}) \leq \sum_{n \in \mathbb{N}}\left(\sum_{a \in A}\left(r_{a}\right)^{R}\right) \cdot\left(r_{\max }\right)^{n R}<\infty$.

Corollary 1.3.5 For the same $k$ as above, for any $\vec{a} \in A^{*}$ and any two points $x, y \in$ $\phi_{\vec{a}}\left(F^{(\infty)}\right), \rho\left(x, y, C_{\vec{a}}\right) \leq\left(r_{\vec{a}}\right)^{R} \cdot k$

Corollary 1.3.6 For $S \in \mathcal{C}$ fixed, there exists a continuous function $\rho_{S}^{\prime}: S \times S \rightarrow \mathbb{R}_{0}^{+}$ whose restriction to $\left(S \cap F^{(\infty)}\right) \times\left(S \cap F^{(\infty)}\right)$ is $\rho(\cdot, \cdot, S)$.

Proof: It is easy to see that if $B \subset B^{\prime}$, then $\rho(x, y, B) \leq \rho\left(x, y, B^{\prime}\right)$ for all $x, y \in B$. That means that if $\left\{\left(x_{n}, y_{n}\right)\right\}$ is Cauchy, $\left\{\rho\left(x_{n}, y_{n}, S\right)\right\}$ is Cauchy. Define $\rho_{S}^{\prime}(x, y)=$ $\lim \rho\left(x_{n}, y_{n}, S\right)$, where $\left(x_{n}, y_{n}\right) \rightarrow(x, y)$.

Let $\rho$ from now on be the completion, as defined above, i.e. $\rho(x, y, S)=\rho_{S}^{\prime}(x, y)$.

Lemma 1.3.7 If $x, y \in F^{(\infty)}$ are elements of two non-neighbouring n-cells, $C_{\vec{a}}$ and $C_{\vec{b}}$, respectively, then $\rho(x, y, \mathbf{K}) \geq\left(r_{\min }\right)^{R \cdot n} / 4 \cdot|A|$.

\section{Proof:}

$$
\begin{aligned}
C(x, y) & \leq \sum_{z \in \phi_{\vec{a}}\left(F^{(0)}\right)} \sum_{w \in \phi_{\vec{d}}\left(F^{(0)}\right)} C(z, w) \\
& \leq \sum_{\substack{\vec{b} \in A^{n}: \\
C_{\vec{b} \cap n C_{\vec{d}} \neq \dot{b}}}}\left(r_{\vec{b}}\right)^{R} \\
& \leq \sum_{z \in F^{(n)} \cap C_{\vec{d}}} 4 \cdot|A| \cdot\left(r_{\min _{1}}\right)^{-R \cdot n}
\end{aligned}
$$

where $C(x, y)=(\rho(x, y))^{-1}$ is the conductance between $x$ and $y$.

Lemma 1.3 .8 Given conditions on the potential function $v$ that $v_{0}=0$ and $v_{1}=1$, then, if $x, y \in F^{(N)}$ for an $N \in^{*} N-N, x \simeq y \Leftrightarrow v_{x} \simeq v_{y}$. 
Proof: $(\Rightarrow): v_{x}-v_{y}=\rho(x, y) \cdot u_{x y}$. But by corollary 1.3.5 $\rho(x, y) \simeq 0$, and $\left|u_{x y}\right| \leq u_{\underline{1} \underline{0}}=(\rho(\underline{1}, \underline{0}))^{-1}<\infty$.

$(\Leftarrow)$ : Given $x, y$, there exists a first $n$ so that $x, y$ are in two non-neighbouring $n$-cells. By 1.3.7 and by 1.3.5, $\rho(x, y, S) \simeq 0$ iff $n$ is infinite. And $n$ is infinite iff $x \simeq y$.

Corollary 1.3.9 For $S$ fixed, $\rho(\cdot, \cdot, S)$ is a metric.

\subsection{Diffusion}

I will need the following notation: Given $n \in \mathrm{N}$, let the points of $F^{(n)}$ be named by underlined variables, i.e. $\underline{k}, \underline{5}, \ldots$. The two elements of $F^{(0)}$ will still be $\underline{0}$ and $\underline{1}$. We define the binary relation $\mathcal{R}_{n}$ on $F^{(n)}$ by letting $\mathcal{R}_{n}(\underline{i}, \underline{j})=T R U E$ iff there is an $n$-cell $C_{\vec{x}}$ such that $\underline{i}, \underline{j} \in C_{\vec{x}}$.

Given different diffusions on $\mathbf{K}$ we get different transition probabilities. Each of these diffusions induce different random walks on different grids on $\mathbf{K}$, where the transition probabilities for the random walks equal the corresponding transition probabilities for the diffusions. As mentioned in the previous section, when these random walks are symmetric, they will correspond to relations between conductances on an electrical network. What we will call the geometric diffusion is the one that corresponds to the geometric electrical network. To put it in more precise terms: If $B(t, \omega)$ is our diffusion, we want

$$
p_{n}(\underline{i}, \vec{x})=\frac{\rho\left(C_{\vec{x}}\right)}{\sum_{\substack{\vec{y} \in A^{n} \\ \text { ind }}} \rho\left(C_{\vec{y}}\right)}=\frac{\left(r_{\vec{y}}\right)^{-R}}{\sum_{\substack{\vec{y} \in A^{n} \\ \dot{j} \in C_{\vec{y}}}}\left(r_{\vec{y}}\right)^{-R}}
$$

where we define functions $p_{n}: F^{(n)} \times A^{n} \rightarrow[0,1]$ by letting $p_{n}(\underline{i}, \vec{x})$ be the probability that the diffusion started in $\underline{i} \in F^{(n)}$ reaches the next element in $F^{(n)}$ by $C_{\vec{x}}$, that is, there exists $\varepsilon>0$ such that for $t$ where $\tau-\varepsilon<t<\tau$, we have $B(t) \in C_{\vec{x}}$. Here $\tau$ designates the first hitting time of $F^{(n)}-\underline{i}$. The $p_{n}$ will be the transition probabilities for the random walk $\left.B(t, \omega)\right|_{F(n)}$.

More than this, if the diffusion is to be called geometric, time should also be scaled geometrically, in the following sense: Let $B(t, \omega)$ be our diffusion. $B(t, \omega)$ scales time geometrically, if

1. Given $\vec{x} \in A^{n}, B\left(t \wedge \tau_{\vec{x}}, \omega\right)$ has the same law as $\phi_{\vec{x}}\left(B\left(\theta\left(C_{\vec{x}}\right) \cdot(t \wedge \tau), \omega\right)\right)$, where $\tau=\min \left\{t \in \mathbf{R}: B(t, \omega) \in F^{(0)}\right\}, \tau_{\vec{x}}=\min \left\{t \in \mathbf{R}: B(t, \omega) \in F^{(n)}\right\}$ and $\theta$ is the function that gives the time scaling factor of the cell as output.

2. $\theta\left(C_{(\cdot)}\right)$ is geometric. 
If $B(t, \omega)$ satisfies both the demand that time scaling is geometric, and that the corresponding electrical network is geometric, $B(t, \omega)$ itself will be called geometric. This will be our Brownian motion.

Lemma 1.4.1 If $B(t, \omega)$ is geometric, then, for the associated $\theta, \theta\left(C_{\vec{a}}\right)=\left(r_{\vec{a}}\right)^{T}$ for some real number $T$

Proof: $\theta\left(C_{(\cdot)}\right)$ satisfies the condition of 1.1 .1 of being geometrical.

We would now like to prove the uniqueness of $T$, together with the existence of the time scaling factor $\theta$. The existence of the time scaling factor, however, is conditional on the existence of the geometric diffusion, which isn't exactly trivially given. So we shall first have to construct the geometric diffusion, which was the purpose of this paper, anyway.

We start by constructing random walks on $F^{(n)}$, with the goal of finding the gometric diffusion as a limit of these. Doing this, we keep the convenient "probability over a cell" function. Let $X_{n}(t)$ be the random walks on $F^{(n)}$. If $C_{\vec{x}}$ is an $n$-cell, $X_{n}\left(t_{0}\right)=\underline{j} \in$ $\phi_{\vec{x}}\left(F^{(0)}\right)$, let $p_{n}(\underline{j}, \vec{x})$ be the probability of $X_{n}$ hitting the next point in $F^{(n)}$ "over" $C_{\vec{x}}$. When $\underline{i}, \underline{j} \in F^{(n)}, \underline{i} \neq \underline{j}$, we get that, given that $X_{n}$ is in $\underline{i}$, the probability that the next point that $X_{n}$ hits in $F^{(n)}$ is $\underline{j}$, is

$$
p_{n}(\underline{j}, \underline{i})=\sum_{\substack{\vec{x} \in A^{n}: \\ \dot{i}, j \in C_{\vec{x}}}} p_{n}(\underline{i}, \vec{x})=\sum_{\substack{\vec{z} \in A^{n}: \\ \underline{i}, \underline{j} \in C_{\vec{x}}}} \frac{\left(r_{\vec{x}}\right)^{-R}}{\sum_{\substack{\vec{j} \in A^{n}: \\ \dot{i} \in C_{\mathfrak{y}}}}\left(r_{\vec{y}}\right)^{-R}}
$$

Let the mean transition time "over" $C_{\vec{x}}$ be $t_{\vec{x}}$, and the mean transition time between $\underline{i}$ and $\underline{j}$ be $t_{\underline{i} \rightarrow \underline{j}}$. Since we want the limit diffusion to have geometric time scaling, we feel we should require, at least as a first approximation, that $t_{\phi_{\vec{a}(\vec{x})}}=\theta(\vec{a}) \cdot t_{\vec{x}}=\left(r_{\vec{a}}\right)^{T} \cdot t_{\vec{x}}$. With $t_{\hat{0}}=t_{\underline{1} \rightarrow \underline{0}}$, that means $t_{\vec{x}}=t_{\underline{1} \rightarrow \underline{0}} \cdot\left(r_{\vec{x}}\right)^{T}$.

We can without loss of generality set $t_{\underline{1} \rightarrow \underline{0}}=1$. Setting up the exact expressions analoguous to the transition probabilities, we have

$$
t_{\underline{i} \rightarrow \underline{j}}=\frac{\sum_{\substack{\vec{x} \in A^{n} \\ \dot{i}, \underline{j} \in C_{\vec{x}}}} t_{\vec{x}} \cdot p_{n}(\underline{j}, \vec{x})}{\sum_{\substack{\vec{x} \in A^{n} \\ \dot{j}, \underline{j} \in C_{\vec{x}}}} p_{n}(\underline{j}, \vec{x})}=\frac{\sum_{\substack{\vec{x} \in A^{n} \\ \dot{i}, j \in C_{\vec{x}}}}\left(r_{\vec{x}}\right)^{T} \cdot\left(r_{\vec{x}}\right)^{-R}}{\sum_{\substack{\vec{x} \in A^{n} \\ i, j \in C_{\vec{x}}}}\left(r_{\vec{x}}\right)^{-R}}
$$

We notice that $t_{\underline{i} \rightarrow \underline{j}}=t_{\underline{j} \rightarrow \underline{i}}$

We will now find, for each $n \in \mathbb{N}$, the required exponent $T$ that, when $t_{\vec{x}}=\left(r_{\vec{x}}\right)^{T}$, yield the expected composite time for $X_{n}$ to go from $\underline{1}$ to $\underline{0}$ to be 1 .

One should not expect a priori, that this $T$ belonging to $X_{n}$ should be the same for all $n$, but it turns out that we have the following result, independent of $n$ : 
Theorem 1.4.2 ${ }^{2} T=R+D$

Proof: Let $t_{\underline{k}}$ be mean transition time for $X_{n}$ from $\underline{k}$ to $\underline{\underline{0}} . t_{\underline{0}}=0$ and $t_{\underline{1}}=1$.

We then have two systems of linear equations to solve. The first one is from our calculation of $R$. Let $v_{\underline{i}}$ be the voltage in $\underline{i}$ given $v_{0}=0$ and $v_{1}=1$, and let $u_{i \underline{j}}$ be the total current flow from $\underline{i}$ to $\underline{j}$ over the resistances (n-cells) between $\underline{i}$ and $\underline{j}$, given the same restrictions on $v$. Fixing $\underline{i}$, we get:

$$
u_{\underline{i j}}=\sum_{C: \underline{i}, \underline{j} \in C} \frac{v_{\underline{i}}-v_{j}}{\rho(C)}, \sum_{\underline{j}: \mathcal{R}_{n}(\underline{i}, \underline{j})} u_{\underline{i} \underline{j}}=0
$$

giving us

$$
\sum_{\underline{j}: \mathcal{R}_{n}(\underline{i}, \underline{j})} \sum_{C: i, \underline{j}, \underline{j} \in C} \frac{v_{\underline{i}}-v_{\underline{j}}}{\rho(C)}=0
$$

and further that

$$
\sum_{C: \underline{i} \in C} \frac{v_{\underline{i}}}{\rho(C)}-\left(\sum_{j: \mathcal{R}_{n}(\underline{\underline{i}}, \underline{j})} \sum_{C: \underline{i}, \underline{j} \in C} \frac{v_{\underline{j}}}{\rho(C)}\right)=0
$$

with an exception for $\underline{i}=\underline{1}$, where with $\sum_{\underline{j}: \mathbb{R}_{n}(\underline{1} \underline{j})} u_{\underline{i} \underline{\underline{j}}}=1$, we get

$$
\sum_{C: \underline{1} \in C} \frac{v_{\underline{1}}}{\rho(C)}-\left(\sum_{j: \mathcal{R}_{n}(\underline{\underline{1}} \underline{\underline{j}})} \sum_{C: \underline{1} \underline{\underline{j}} \in C} \frac{v_{\underline{j}}}{\rho(C)}\right)=1
$$

This is a system of equations to be solved for $v_{\underline{i}}$. We get the matrix $M$ for the equation by letting the matrix elements be

$$
m_{\underline{i j}}=m_{\underline{j i}}=-\sum_{C: \underline{i}, \underline{j} \in C} \frac{1}{\rho(C)} \text { for } \underline{i} \neq \underline{j}, \text { and } m_{\underline{i i}}=\sum_{C: \underline{i} \in C} \frac{1}{\rho(C)}
$$

Let $\vec{v}=\left[v_{\underline{1}}, v_{\underline{2}}, \ldots, v_{\underline{n}}\right]$, and $\vec{u}=[1,0, \ldots, 0]$.

Then $\mathbf{M} \cdot \vec{v}=\vec{u}$

We also want to find the system of equations for mean transition time:

For each point we have that mean transition time from the point $\underline{k}$ to $\underline{0}, t_{\underline{k}}$, equals the mean of (neighbour's mean transition time + mean transition time to same):

$$
t_{\underline{k}}=\sum_{\underline{j}: \mathbb{R}_{n}(\underline{k}, \underline{j})} p_{n}(\underline{k}, \underline{j})\left[t_{\underline{k} \rightarrow \underline{j}}+t_{\underline{j}}\right]
$$

\footnotetext{
${ }^{2}$ This is known as The Einstein relation
} 
or, if we put in the values of $p_{n}$ and $t_{\underline{j} \rightarrow \underline{k}}$ :

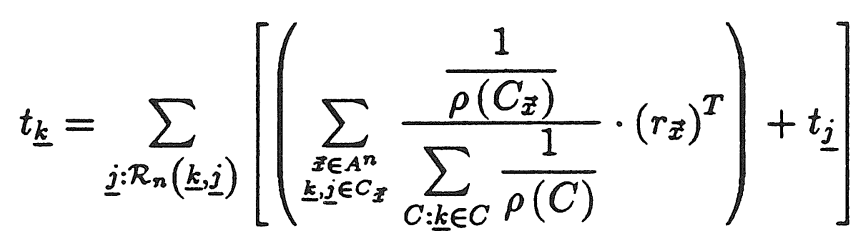

which is

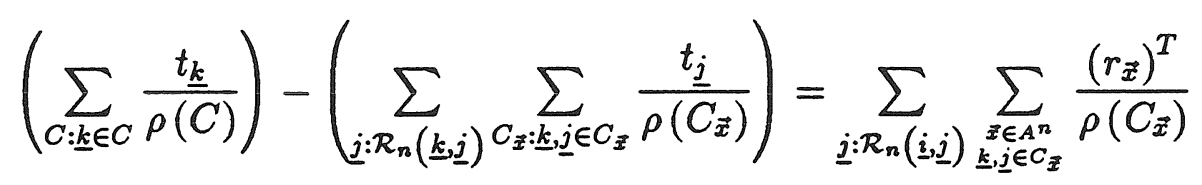

or in matrix form

$$
\mathrm{M} \cdot \vec{t}=\vec{T}
$$

where

$$
\vec{t}=\left[t_{1}, \underline{t_{2}}, \ldots, t_{\underline{n}}\right], \text { and } \vec{T}=\left[\sum_{\substack{\vec{x} \in A^{n} \\ \underline{x} \in C_{\vec{x}}}} \frac{\left(r_{\vec{x}}\right)^{T}}{\rho\left(C_{\vec{x}}\right)}, \sum_{\substack{x \in A^{n} \\ \underline{z} \in C_{\vec{x}}}} \frac{\left(r_{\vec{x}}\right)^{T}}{\rho\left(C_{\vec{x}}\right)}, \ldots, \sum_{\substack{\vec{x} \in A^{n} \\ n \in C_{\vec{x}}}} \frac{\left(r_{\vec{x}}\right)^{T}}{\rho\left(C_{\vec{x}}\right)}\right]
$$

or, if we remember that $\rho\left(C_{\vec{x}}\right)=\left(r_{\vec{x}}\right)^{R}$,

$$
\vec{T}=\left[\sum_{\substack{\vec{x} \in A^{n} \\ \underline{1} \in C_{\vec{X}}}}\left(r_{\vec{x}}\right)^{T-R}, \sum_{\substack{\vec{z} \in A^{n} \\ \underline{2} \in C_{\vec{X}}}}\left(r_{\vec{x}}\right)^{T-R}, \ldots, \sum_{\substack{\vec{x} \in A^{n} \\ \underline{n} \in C_{\vec{X}}}}\left(r_{\vec{x}}\right)^{T-R}\right]
$$

Let $[\text { vector }]_{i}$ be the $i$ 'th component of [vector]. We will apply Cramer's Rule from linear algebra, stating that for a system of equations $\mathbf{A} \cdot \vec{x}=\vec{y}$, we have $x_{i}=\frac{\operatorname{Det}\left(\mathbf{A}_{\mathbf{i}}\right)}{\operatorname{Det}(\mathbf{A})}$, where $\mathbf{A}_{\mathbf{i}}$ is the matrix we get by replacing the i'th coloumn of $\mathbf{A}$ with $\vec{y}$.

The first system of equations gives us that $v_{\underline{k}}=\frac{\operatorname{Det}\left(\mathbf{M}_{\mathbf{k}}\right)}{\operatorname{Det}(\mathbf{M})}$, where $\mathbf{M}_{\mathbf{k}}$ is $\mathbf{M}$ with the k'th coloumn replaced by $\vec{u}$. But as $u_{1}=1$ and $u_{\vec{x}}=0$ for $i \neq 1$, we get that $\operatorname{Det}\left(M_{k}\right)=$ $\operatorname{Det}\left(C_{1 k}\right)$, which since $m_{i j}=m_{j i}$, again equals $\operatorname{Det}\left(C_{k 1}\right)$, where $C_{i j}$ is the cofactor of $m_{i j}$.

But by using the same Cramer's Rule, we get that $t_{\underline{i}}=\frac{\operatorname{Det}\left(\mathbf{M}_{\mathbf{i}}^{\prime}\right)}{\operatorname{Det}(\mathbf{M})}$, where $\mathbf{M}_{\mathbf{i}}^{\prime}$ is $\mathbf{M}$ with the i'te coloumn replaced by $\vec{T}$. We have in particular that

$$
1=t_{\underline{1}}=\frac{\operatorname{Det}\left(\mathbf{M}_{1}^{\prime}\right)}{\operatorname{Det}(\mathbf{M})}
$$

where

$$
\operatorname{Det}\left(\mathbf{M}_{1}^{\prime}\right)=\sum_{i=1}^{n} T_{\vec{x}} \cdot \operatorname{Det}\left(C_{i 1}\right)
$$


which then gives us

$$
\begin{gathered}
1=\frac{\operatorname{Det}\left(\mathbf{M}_{1}^{\prime}\right)}{\operatorname{Det}(\mathbf{M})}=\sum_{i=1}^{n} T_{\vec{x}} \cdot \frac{\operatorname{Det}\left(C_{1 i}\right)}{\operatorname{Det}(\mathbf{M})}=\sum_{i=1}^{n} T_{\vec{x}} \cdot v_{\underline{i}} \\
\sum_{k=1}^{n} T_{k} \cdot v_{\underline{k}}=1
\end{gathered}
$$

For each cell $C_{\vec{x}}$ there are exactly two points, $\underline{k}(\vec{x})$ and $\underline{l}(\vec{x})$, and with $v_{0}=0$, we have

$$
\sum_{\vec{x} \in A^{n}}\left(\left(r_{\vec{x}}\right)^{T-R} \cdot\left(v_{\underline{k}(\vec{x})}+v_{\underline{l}(\vec{x})}\right)\right)=1
$$

It is easy to see that if $U(\underline{k})=\underline{j}$, then $v_{\underline{k}}=1-v_{\underline{j}}$. We split the cells into two categories: The $C$ where $U(C)=C$, and the rest;

$\sum_{\vec{x} \in A^{n}: C_{\vec{x}}=U\left(C_{\vec{x}}\right)}\left(r_{\vec{x}}\right)^{T-R}+\sum_{\vec{x} \in A^{n}: C_{\vec{x}} \neq U\left(C_{\vec{x}}\right)} \frac{1}{2}\left(\left(r_{\vec{x}}\right)^{T-R}\left[\left(v_{\underline{k}(\vec{x})}+v_{\underline{l}(\vec{x})}\right)+\left(1-v_{\underline{k}(\vec{x})}+1-v_{\underline{l}(\vec{x})}\right)\right]\right)=1$

which, when we collect the terms:

$$
\sum_{\vec{x} \in A^{n}}\left(r_{\vec{x}}\right)^{T-R}=1
$$

By uniqueness of this exponent (lemma 1.2.1), $T-R=D$ 


\section{Chapter 2}

\section{Brownian Motion on Simple Fractal Spaces}

The random walks given in the previous chapter will have as their limit a strong Markov process with a.s. continuous paths, which scales geometrically. To show this, we must define our random walks in more detail, so that we can apply some technical machinery to our proofs. In the following section we will define our technical machinery.

\subsection{Some lemmas on $\mathcal{G}$}

In this section we will look at some measures. These will be the set of completed Borel probability measures on $[0, \infty]$ with finite first and second moments, and will be denoted by $\mathcal{G}$. Lindstrøm [12] has constructed a metric on $\mathcal{G}$, letting

$$
\begin{array}{r}
d(\sigma, \tau)=\inf \left\{\left(\int_{\Omega}|f-g|^{2} d P\right)^{\frac{1}{2}}:(\Omega, P)\right. \text { probability space, and } \\
f, g: \Omega \rightarrow[0, \infty] \text { r.v.s with distributions resp. } \sigma \text { and } \tau\}
\end{array}
$$

and shown that $(\mathcal{G}, d)$ is complete. If we extend $d$ to be defined for arbitrary pairs of distributions of variables $X: \Omega \rightarrow[0, \infty]$ with finite expectation, we have the useful equivalence $\sigma \in \mathcal{G} \Leftrightarrow d\left(\sigma, \sigma_{0}\right)<\infty$, where $\sigma_{0}\{0\}=1$.

For convenience, we will let $\sigma_{X}$ denote the distribution of the r.v. $\mathrm{X}$, and we will write $\|\sigma\|$ for $d\left(\sigma, \sigma_{0}\right)$.

Let $B$ be any set, $D \subset B$ countable, and let $N_{X}, N_{Y}$ be independent random variables taking values in $B$, with $\operatorname{Prob}\left\{N_{X}=\alpha\right\}=\operatorname{Prob}\left\{N_{Y}=\alpha\right\}=p_{\alpha}$. Let $\left\{X_{\alpha}\right\}_{\alpha \in B}$ and $\left\{Y_{\alpha}\right\}_{\alpha \in B}$ be random variables, independent of $N_{X}$ and $N_{Y}$. If we now let $X=X_{N_{X}}$, and $Y=Y_{N_{Y}}$, we have two new random variables. 
Lemma 2.1.1 With variables defined as above, when $X_{\alpha} \in \mathcal{G}$ for all $\alpha \in D$, and $\sum_{\alpha \in D} p_{\alpha}=1$, we have

1. If

(a) $\sum_{\alpha \in D} p_{\alpha} \cdot E\left[X_{\alpha}\right]<\infty$

(b) $\sum_{\alpha \in D} p_{\alpha} \cdot\left\|\sigma_{X_{\alpha}}\right\|^{2}<\infty$

then $\sigma_{X} \in \mathcal{G}$.

2. $\left(d\left(\sigma_{X}, \sigma_{Y}\right)\right)^{2} \leq \sum_{\alpha \in D} p_{\alpha} \cdot\left(d\left(\sigma_{X_{\alpha}}, \sigma_{Y_{\alpha}}\right)\right)^{2}$

\section{Proof: 2. is}

$\left(d\left(\sigma_{X}, \sigma_{Y}\right)\right)^{2}=\inf \left\{\int_{\Omega}|f-g|^{2} d P:(\Omega, P)\right.$ probability space, $\left.\sigma_{f}=\sigma_{X}, \sigma_{g}=\sigma_{Y}\right\}$

$\leq \inf \left\{\int_{\Omega}\left|f_{N(\omega)}-g_{N(\omega)}\right|^{2} d P:(\Omega, P)\right.$ probability space, $N: \Omega \rightarrow B$, with $P(N=\alpha)=p_{\alpha}$ and $f_{\alpha}, g_{\alpha}$ independent of $\left.N . \sigma_{f_{\alpha}}=\sigma_{X_{\alpha}}, \sigma_{g_{\alpha}}=\sigma_{Y_{\alpha}}\right\}$

$=\inf \left\{\int_{\Omega} \sum_{\alpha \in B} \chi_{\alpha}(N)\left|f_{\alpha}-g_{\alpha}\right|^{2} d P:(\Omega, P)\right.$ probability space, $N: \Omega \rightarrow B$, with $P(N=\alpha)=p_{\alpha}$ and $f_{\alpha}, g_{\alpha}$ independent of $\left.N . \sigma_{f_{\alpha}}=\sigma_{X_{\alpha}}, \sigma_{g_{\alpha}}=\sigma_{Y_{\alpha}}\right\}$

$=\inf \left\{\sum_{\alpha \in B} \int_{\Omega_{\alpha}}\left|f_{\alpha}-g_{\alpha}\right|^{2} d P:(\Omega, P)\right.$ probability space, $\left\{\Omega_{\alpha}\right\}_{\alpha \in B}$ disjoint covering of $\Omega$, with $P\left(\Omega_{\alpha}\right)=p_{\alpha}$ and $f_{\alpha}, g_{\alpha}$ independent of $\left.\left\{\Omega_{\alpha}\right\}_{\alpha \in B} . \sigma_{f_{\alpha}}=\sigma_{X_{\alpha}}, \sigma_{g_{\alpha}}=\sigma_{Y_{\alpha}}\right\}$ $=\inf \left\{\sum_{\alpha \in D} \int_{\Omega_{\alpha}}\left|f_{\alpha}-g_{\alpha}\right|^{2} d P:(\Omega, P)\right.$ probability space, $\left\{\Omega_{\alpha}\right\}_{\alpha \in D}$ disjoint covering of $\Omega$, with $P\left(\Omega_{\alpha}\right)=p_{\alpha}$ and $f_{\alpha}, g_{\alpha}$ independent of $\left.\left\{\Omega_{\alpha}\right\}_{\alpha \in D}, \sigma_{f_{\alpha}}=\sigma_{X_{\alpha}}, \sigma_{g_{\alpha}}=\sigma_{Y_{\alpha}}\right\}$ $=\sum_{\alpha \in D} p_{\alpha} \cdot \inf \left\{\int_{\Omega}\left|f_{\alpha}-g_{\alpha}\right|^{2} d P:(\Omega, P)\right.$ probability space, and $\left.\sigma_{f_{\alpha}}=\sigma_{X_{\alpha}}, \sigma_{g_{\alpha}}=\sigma_{Y_{\alpha}}\right\}$ $=\sum_{\alpha \in D} p_{\alpha} \cdot\left(d\left(\sigma_{X_{\alpha}}, \sigma_{Y_{\alpha}}\right)\right)^{2}$

where $\chi_{\alpha}(N)= \begin{cases}1 & \text { if } N=\alpha \\ 0 & \text { otherwise }\end{cases}$

To prove 1., notice that

$$
E[X]=\sum_{\alpha \in B} p_{\alpha} \cdot E\left[X_{\alpha}\right]=\sum_{\alpha \in D} p_{\alpha} \cdot E\left[X_{\alpha}\right]<\infty
$$


and that by 2 .

$$
\left\|\sigma_{X}\right\|^{2} \leq \sum_{\alpha \in D} p_{\alpha} \cdot\left\|\sigma_{X_{\alpha}}\right\|^{2}<\infty
$$

Given a random variable $X$, we can simply construct a new variable by multiplying by a constant $\beta$. For a distribution $\sigma$, similarly, we can construct a new distribution $\beta \diamond \sigma$ by letting $(\beta \diamond \sigma)(B)=\sigma(\{x \in R: \beta \cdot x \in B\})$. It is obvious that $\beta \diamond \sigma_{X}=\sigma_{\beta X}$.

Lemma 2.1.2 Given two random variables $X, Y$ and a constant $\beta$, then we have

1. $\left\|\sigma_{\beta X}\right\|=|\beta| \cdot\left\|\sigma_{X}\right\|$, so if $\beta \neq 0$, then $\sigma_{X} \in \mathcal{G} \Leftrightarrow \sigma_{\beta X} \in \mathcal{G}$.

2. $d\left(\sigma_{\beta X}, \sigma_{\beta Y}\right)=|\beta| \cdot d\left(\sigma_{X}, \sigma_{Y}\right)$

Proof: The first follow from the second, and for the second, we have

$$
\begin{aligned}
d\left(\sigma_{\beta X}, \sigma_{\beta Y}\right) & =\inf \left\{\left(\int_{\Omega}|f-g|^{2} d P\right)^{\frac{1}{2}}:(\Omega, P) \text { probability space, } \sigma_{f}=\sigma_{\beta X}, \sigma_{g}=\sigma_{\beta Y}\right\} \\
& =\inf \left\{\left(\int_{\Omega}|\beta f-\beta g|^{2} d P\right)^{\frac{1}{2}}:(\Omega, P) \text { probability space, } \sigma_{\beta f}=\sigma_{\beta X}, \sigma_{\beta g}=\sigma_{\beta Y}\right\} \\
& =\inf \left\{|\beta|\left(\int_{\Omega}|f-g|^{2} d P\right)^{\frac{1}{2}}:(\Omega, P) \text { probability space, } \sigma_{f}=\sigma_{X}, \sigma_{g}=\sigma_{Y}\right\} \\
& =|\beta| \cdot d\left(\sigma_{X}, \sigma_{Y}\right)
\end{aligned}
$$

Given a finite number of random variables, we can make a new random variable simply by adding the existing variables. Let $\left\{X_{n}\right\}_{n \in I}$ and $\left\{Y_{n}\right\}_{n \in I}$ where $I$ is some finite set, be positive random variables, and let $X=\sum_{I} X_{n}$ and $Y=\sum_{I} Y_{n}$.

Lemma 2.1.3 If the $X_{i}$ and the $Y_{i}$, as described above, are independent, then

1. $\left\|\sigma_{X}\right\|^{2} \leq \sum_{I}\left\|\sigma_{X_{n}}\right\|^{2}+\left(\sum_{I} E\left[X_{n}\right]\right)^{2}$, so if $\sigma_{X_{n}} \in \mathcal{G}$ for all $n$, then $\sigma_{X} \in \mathcal{G}$.

2. $\left(d\left(\sigma_{X}, \sigma_{Y}\right)\right)^{2} \leq \sum_{I}\left(d\left(\sigma_{X_{n}}, \sigma_{Y_{n}}\right)\right)^{2}+\left(\sum_{I} E\left[X_{n}\right]-E\left[Y_{n}\right]\right)^{2}$

3. If $E\left[X_{n}\right]=E\left[Y_{n}\right]$, then $E[X]=E[Y]$ and $\left(d\left(\sigma_{X}, \sigma_{Y}\right)\right)^{2} \leq \sum_{I}\left(d\left(\sigma_{X_{n}}, \sigma_{Y_{n}}\right)\right)^{2}$ 
Proof: The last part is trivial. And the first part is just going through our computational machinery:

$$
\begin{aligned}
\left(d\left(\sigma_{X}, \sigma_{Y}\right)\right)^{2}= & \inf \left\{\int_{\Omega}|f-g|^{2} d P:(\Omega, P) \text { probability space, } \sigma_{f}=\sigma_{X}, \sigma_{g}=\sigma_{Y}\right\} \\
= & \inf \left\{\int_{\Omega}\left|\sum_{I} f_{n}-\sum_{I} g_{n}\right|^{2} d P:(\Omega, P)\right. \text { probability space, and } \\
& \left.\sigma_{\sum_{f_{i}}}=\sigma_{\sum X_{i}}, \sigma_{\sum_{g_{i}}}=\sigma_{\sum Y_{i}}\right\} \\
\leq & \inf \left\{\int_{\Omega}\left|\sum_{I}\left(f_{n}-g_{n}\right)\right|^{2} d P:(\Omega, P) \text { probability space, } \sigma_{f_{i}}=\sigma_{X_{i}}, \sigma_{g_{i}}=\sigma_{Y_{i}}\right. \\
& \left.\operatorname{and} f_{i} \text { and } g_{i} \text { independent }\right\} \\
= & \inf \left\{E\left[\left|\sum_{n}\left(f_{n}-g_{n}\right)\right|^{2}\right]:(\Omega, P) \text { probability space, } \sigma_{f_{i}}=\sigma_{X_{i}}, \sigma_{g_{i}}=\sigma_{Y_{i}}\right. \\
& \left.\operatorname{and} f_{i} \text { and } g_{i} \text { independent }\right\} \\
= & \inf \left\{\sum_{I} E\left[\left(f_{n}-g_{n}\right)^{2}\right]+\sum_{n \in I} \sum_{\substack{m \in I \\
m \neq n}} E\left[\left(f_{n}-g_{n}\right)\right] \cdot E\left[\left(f_{m}-g_{m}\right)\right]:\right. \\
& \left.(\Omega, P) \text { probability space, } \sigma_{f_{i}}=\sigma_{X_{i}}, \sigma_{g_{i}}=\sigma_{Y_{i}}, f_{i} \text { and } g_{i} \text { independent }\right\} \\
= & \inf \left\{\sum_{I} E\left[\left(f_{n}-g_{n}\right)^{2}\right]+\left(\sum_{n \in I} E\left[\left(f_{n}-g_{n}\right)\right]\right)^{2}-\sum_{n \in I} E\left[\left(f_{n}-g_{n}\right)\right]^{2}:\right. \\
& \left.(\Omega, P) \text { probability space, } \sigma_{f_{i}}=\sigma_{X_{i}}, \sigma_{g_{i}}=\sigma_{Y_{i}}, f_{i} \text { and } g_{i} \text { independent }\right\} \\
\leq & \inf \left\{\sum_{I} E\left[\left(f_{n}-g_{n}\right)^{2}\right]+\left(\sum_{n \in I} E\left[\left(f_{n}-g_{n}\right)\right]\right)^{2}:(\Omega, P)\right. \text { probability space, } \\
& \left.\sigma_{f_{i}}=\sigma_{X_{i}}, \sigma_{g_{i}}=\sigma_{Y_{i}} f_{i} \text { and } g_{i} \text { independent }\right\} \\
\leq & \sum_{I}\left(d\left(\sigma_{X_{n}}, \sigma_{Y_{n}}\right)\right)^{2}+\left(\sum_{I} E\left[X_{n}\right]-E\left[Y_{n}\right]\right)^{2}
\end{aligned}
$$

I will write $E[\tau]$ for $\int x d \tau$. If $\tau([0, t]) \leq \sigma([0, t])$ for all $t$, I write $\tau \geq \sigma$.

Lemma 2.1.4 Let $\psi_{k}$ is an increasing sequence of random variables, with $\psi_{0}=0$, and let $\sigma_{k}$ be the distribution of $\Delta \psi_{k}=\psi_{k}-\psi_{k-1}$. If there exists some fixed distribution $\sigma$ with $E[\sigma]>0$, such that for each $k, \sigma_{k} \geq \sigma$, then

$$
P\left\{\omega \in \Omega: \exists k(\omega) \in \mathbb{N} \text { such that } \psi_{k(\omega)}>r\right\}=1, \forall r \in \mathbb{R}
$$

Proof: Let $p=\sigma([0, E[\sigma]])$. Fix $r \in \mathbb{R}$. Let $A_{k}=\left\{\omega: \psi_{k} \leq r\right\}$. We see that if $\psi_{k} \leq r$, then we must have $\Delta \psi_{l} \leq E[\sigma]$ for at least $n$ natural numbers $l, l \leq k$, where $n=k-\left[\left[\frac{r}{E[\sigma]}\right]\right]$. Thus, $P\left\{A_{k}\right\} \leq p^{a}$. Since now $\sum A_{k}<\infty$, we can use Borel-Cantelli, 
and get that for almost all $\omega$ there exists a $k(\omega) \in N$ such that $\omega \notin A_{l}$ for any $l \geq k(\omega)$.

This lemma is the one that assures us that we can speak confidently of the processes we are working with as being defined for all $t \in \mathbb{R}^{+}$.

\subsection{Random walks}

We will now carry out the detailed construction of the random walks mentioned in chapter 1. There is one problem, however, which is that we do not know the distribution of the transition time from 1 to $\underline{0}$. But, as we shall show in the next section, knowing the distribution is not necessary in order to find the limit Brownian motion. So we will just fix a completed Borel probability measure $\tau$ on $[0, \infty]$, with $\int x d \tau=1$ and $\int x^{2} d \tau<\infty$, and call the associated random walks the random walks induced by $\tau$.

Let $B^{(n)}$ be a Markov chain on $F^{(n)}$ with the transition probabilities given in the previous chapter, with the additional property that it goes from point to point "over" a certain $n$-cell. We write $C^{(n)}(k)$ for the cell $B^{(n)}$ passes over from $B^{(n)}(k-1)$ to $B^{(n)}(k)$.

Then let $T_{k}^{(n)}$ be an increasing sequence of random variables for each $n$, satisfying

1. $T_{0}^{(n)}=0$

2. $\Delta T_{k}^{(n)}=T_{k}^{(n)}-T_{k-1}^{(n)}$ :

(a) $E\left[\Delta T_{k}^{(n)} \mid B^{(n)}(k-1)=\underline{i}, B^{(n)}(k)=\underline{j}, C^{(n)}(k)=C_{\vec{a}}\right]=t_{\vec{a}}$

(b) Take $\Delta T_{k}^{(n)}$ given $B^{(n)}(k-1)=\underline{i}, B^{(n)}(k)=\underline{j}$ and $C^{(n)}(k)=C_{\vec{a}}$.

Then $\sigma_{\Delta T_{k}^{(n)}}=t_{\vec{a}} \diamond \tau$

3. The $\left.\Delta T_{k}^{(n)}\right|_{B^{(n)}(k-1)=i, B^{(n)}(k)=\underline{j}, C^{(n)}(k)=C_{\vec{a}}}$ are independent of each other and each of them conditionally independent of $\left.C^{(n)}(k)\right|_{B^{(n)}(k-1)}$.

We now define our random walks:

$$
X_{\tau}^{(n)}(t, \omega)=B^{(n)}(k, \omega), k=\max \left\{i: T_{i}^{(n)}(\omega) \leq t\right\}
$$

By lemma 2.1.4 these are well-defined, a.s. The $X_{\tau}^{(n)}$ will be the random walks $\tau$ induces on $F^{(n)}$

Now, let $W_{\tau, m}^{(n)}$ be the random walks induced on $F^{(n)}$ by $X_{\tau}^{(m)}, m>n$;

$$
W_{\tau, m}^{(n)}(t, \omega)=X_{\tau}^{(m)}\left(t^{\prime}, \omega\right), \text { where } t^{\prime}=\max \left\{t^{\prime} \leq t: X_{\tau}^{(m)}\left(t^{\prime}, \omega\right) \in F^{(n)}\right\}
$$


We say that $W_{\tau, m}^{(n)}$ go from $\underline{i}$ to $\underline{j}$ via $C_{\vec{a}}, \vec{a} \in A^{n}$, at time $t$ if $W_{\tau, m}^{(n)}(t, \omega)=\underline{j}$, and there exists an $\varepsilon>0$ such that for all $\tilde{t}: t-\varepsilon<\tilde{t}<t, W_{\tau, m}^{(n)}(\tilde{t}, \omega)=\underline{i}$, and $X_{\tau}^{(m)}(\tilde{t}, \omega) \in C_{\vec{a}}$. That is, given $\tau$ fixed, we let

$$
\Theta_{l, m}^{(n)}(\omega)=T_{k_{l}}^{(m)}(\omega) \text {, where } k_{l}=\min \left\{k>k_{l-1}: X^{(n)}\left(T_{k_{l}}^{(m)}\right) \in F^{(m)}-F^{(n)}\right\}
$$

So, $\left\{\Theta_{k, m}^{(n)}\right\}_{k \in N_{0}}$ is a sequence of random variables increasing with $k$ such that

1. $\Theta_{0, m}^{(n)}=T_{k_{0}}^{(m)}$

2. If $\Delta \Theta_{k, m}^{(n)}=\Theta_{k, m}^{(n)}-\Theta_{k-1, m}^{(n)}$, then the distribution of $\left.\Delta \Theta_{k, m}^{(n)}\right|_{B^{(n)}(k-1)=\underline{i}, B^{(n)}(k)=\underline{j}, C^{(n)}(k)=C_{\bar{d}}}$ equals the distribution of the hitting time for $X_{\tau}^{(m)}$ given that it starts in $\underline{i}$ and hits $\underline{j}$ via $C_{\vec{a}}$ before it hits any other element of $F^{(n)}-\underline{i}$.

3. The $\left.\Delta \Theta_{k, m}^{(n)}\right|_{B^{(n)}(k-1)=i, B^{(n)}(k)=j, C^{(n)}(k)=C_{\bar{\delta}}}$ are independent of each other and each of them conditionally independent of $\left.C^{(n)}(k)\right|_{B^{(n)}(k-1)}$

We see from the definition of $W$ that

$$
W_{\tau, m}^{(n)}(t, \omega)=B^{(n)}(k, \omega), k=\max \left\{i: \Theta_{i, m}^{(n)}(\omega) \leq t\right\}
$$

We also here omit the $\tau$ where it is redundant given the context. To get on to the limit of these walks, we need the following lemma:

Lemma 2.2.1 $\sigma_{\Delta \Theta_{k, m}^{(n)}} \in \mathcal{G}$.

Proof: Fix $n$. Given a point $x \in F^{(n)}$, and $m>n$, we want to find the probability that $B^{(m)}$ hits a point in $F^{(n)}-\{x\}$ in less than a given number of steps. For that purpose, we introduce the $n$-neighbourhood of $x, U_{x}^{(n)}$, which consists of those $n$-cells of which $x$ is a member. The boundary of this set, $\delta_{x}$, is a subset of $F^{(n)}$ : As we are interested in $B^{(m)}$ on $U_{x}^{(n)}$, we need to define the $m$-inner of $U_{x}^{(n)}, \tilde{U}=\left(U_{x}^{(n)}-\delta_{x}\right) \cap F^{(m)}$.

We want to find the probability that $B^{(m)}$ has hit $\delta_{x}$ in $k$ steps or less, given that it starts in a point $y \in \tilde{U}$. This is $p_{k, y}=P\left\{\exists l \leq k\left(B^{(m)}(l) \in \delta_{x}\right) \mid B^{(m)}(0)=y\right\}$. Now, if $k_{x}=\min \left\{k \in N: p_{k, y}>0, \forall y \in \tilde{U}\right\}$, then $p_{k_{x}, y}>0 \forall y \in \tilde{U}$. Let $p_{k}=\min \left\{p_{k, y} \mid y \in \tilde{U}\right\}$, and $q_{k}=1-p_{k}$. Then, if $r$ is any positive integer, $q_{r \cdot k_{x}} \leq\left(q_{k_{x}}\right)^{r}$. Thus, there exist constants $Q$ and $q$ such that $q_{k} \leq Q \cdot q^{k}$ for all $k$. That implies, with the same constants

$$
P\left\{\neg \exists l \leq k\left(B^{(m)}(l) \in \delta_{x}\right) \mid B^{(m)}(0)=x\right\} \leq Q \cdot q^{k}
$$

Now, if $X, Y$ are random variables, and $Y \geq X$, we see that $\sigma_{Y} \geq \sigma_{X}$, and thereby $\left\|\sigma_{Y}\right\| \geq\left\|\sigma_{X}\right\|$. And by 2.1 .2 , if $t_{\vec{a}}=\max \left\{t_{\vec{a}}: \vec{a} \in A^{m}\right\}$, then $\left\|\tau_{\vec{g}}\right\| \geq\left\|\tau_{\vec{a}}\right\|$ for all $\vec{a} \in A^{m}$. 
Let $\Pi_{x}$ be the set of all paths of $B^{(m)}$ that start in $x$, and either end in $\delta_{x}$, or continue indefinitely without ever hitting $\delta_{x}$ at all. For each path $\pi \in \Pi_{x}$, let $T(\pi)$ be the time required for $X^{(n)}$ to run through the path, and $p_{\pi}$ the probability of the path. We see that the finite paths make a countable set, and that the probability that the path has infinite length is 0 , so we can apply 2.1.1.

$$
\begin{aligned}
E\left[\Delta \Theta_{k, m}^{(n)}\right] & =\sum_{\pi \in \Pi} E[T(\pi)]=\sum_{\pi \in \Pi} p_{\pi} \cdot \sum_{\vec{a} \in \pi} t_{\vec{a}} \\
& \leq\left(t_{\max _{1}}\right)^{m-1} \cdot \sum_{\pi \in \Pi} p_{\pi} \cdot \sum_{\vec{a} \in \pi} t_{s m-1}=\left(t_{\max _{1}}\right)^{m-1}
\end{aligned}
$$

The last equality follows from the probability distribution of the paths plus the fact that the mean transition time between $\underline{1}$ and $\underline{0}$ is 1 . Let $\ell(\pi)=\sum_{\vec{a} \in \pi} 1$, i.e. the length of $\pi$.

$$
\begin{aligned}
\|T(\pi)\|^{2} & \leq \sum_{\vec{a} \in \pi}\left\|t_{\vec{a}} \diamond \tau\right\|^{2}+\left(\sum_{\vec{a} \in \pi} t_{\vec{a}}\right)^{2} \text { by } T^{(n)} \text { 's property 3., and by } 2.1 .3 \\
& \leq \sum_{\vec{a} \in \pi} t_{\vec{a}}^{2} \cdot\|\tau\|^{2}+\left(\ell(\pi) \cdot\left(t_{\max _{1}}\right)^{m}\right)^{2} \text { by } 2.1 .2 \text { and by } 2.1, \text { so } \\
\left\|\sigma_{\Delta \Theta_{k, m}^{(n)}}\right\|^{2} & \leq \sum_{\pi \in \Pi} p_{\pi} \cdot\|T(\pi)\|^{2} \\
& \leq \sum_{\pi \in \Pi} p_{\pi} \cdot\left(\sum_{\vec{a} \in \pi} t_{\vec{a}}^{2} \cdot\|\tau\|^{2}+\left(\ell(\pi) \cdot\left(t_{\max _{1}}\right)^{m}\right)^{2}\right) \\
& \leq\left(\left(t_{\max _{1}}\right)^{m} \cdot\|\tau\|^{2} \sum_{\pi \in \Pi} p_{\pi} \cdot \sum_{\vec{a} \in \pi} t_{\vec{a}}\right)+\left(\sum_{l=1}^{\infty}\left(q_{l}-q_{l+1}\right) \cdot\left(l \cdot\left(t_{\max _{1}}\right)^{m}\right)^{2}\right) \\
& \leq\left(t_{\max _{1}}\right)^{m} \cdot\|\tau\|^{2} \cdot\left(t_{\max _{1}}\right)^{m-1}+\left(t_{\max _{1}}\right)^{2 m} \cdot\left(\sum_{l=1}^{\infty} Q \cdot q^{l} \cdot l^{2}\right) \\
& \leq\left(t_{\max _{1}}\right)^{2 m-1} \cdot\left(\|\tau\|^{2}+Q \cdot \frac{2 q^{2}}{(1-q)^{3}}\right)
\end{aligned}
$$

What we will now look at, are the transition time distributions on $F^{(1)}$ and $F^{(2)}$ induced by the various $n$-walks. That is, we are considering the $W_{\tau, n}^{(1)}$ and the $W_{\tau, n}^{(2)}$ for all $n \in \mathbb{N}$.

Since our walks go from one element of $F^{(n)}$ to the next over some specific $n$-cell not necessarily uniquely determined by the two elements of $F^{(n)}$, we have a triplet describing each step, $(\underline{k}, \underline{l}, \vec{a})$, where $\underline{k} \in F^{(n)}$ is the element the step goes from, $\underline{l} \in F^{(n)}$ is the element the step goes to, and $C_{\vec{a}}, \vec{a} \in A^{n}$, is the cell the step goes over. We name the set of such triplets $\mathcal{J}^{(n)}$. We write $\mathcal{J}$ for $\mathcal{J}^{(\mathfrak{l})}$, and for $\vec{b} \in A^{n}$, we let $f_{\vec{b}}(\underline{k}, \underline{l}, \vec{a})=\left(f_{\vec{b}}(\underline{k}), f_{\vec{b}}(\underline{l}), \vec{b} \oplus \vec{a}\right)$

Because of the Markov property of the $B^{(n)}$, the $\left.\Delta \Theta_{s, m}^{(n)}\right|_{B^{(n)}(s-1)=\underline{i}, B^{(n)}(s)=\underline{j}, C^{(n)}(s)=C_{\bar{d}}}$ are dependent only on the triplet $(\underline{k}, \underline{l}, \vec{a})$ and not on $s$. Thus, we have a finite set of distributions for each $n$ and $m$. For the distribution of $\left.\Delta \Theta_{s, m}^{(n)}\right|_{B^{(n)}(s-1)=\underline{i}, B^{(n)}(s)=\underline{j}, C^{(n)}(s)=C_{\tilde{d}}}$, 


\section{CHAPTER 2. BROWNIAN MOTION ON SIMPLE FRACTAL SPACES}

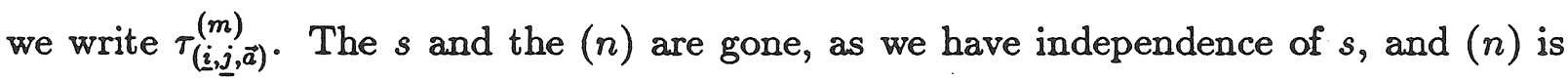
redundant, given $\underline{i}, \underline{j}$ and $\vec{a}$.

While we clearly can express the transition times on $F^{(n)}$ as a composition of the transition times on $F^{(m)}$ when $m \geq n$, we also have relations the other way, in particular one relating the transition times on $F^{(n)}$ to the transition times on $F^{(1)}$. Given some $n \in \mathbb{N}$ and a $j=(\underline{k}, \underline{l}, \vec{a}) \in \mathcal{J}^{(n)}$, then for each $c \in A$, and each $m \in \mathbb{N}, m \geq n$,

$$
\tau_{f_{c}(j)}^{(m+1)}=t_{f_{c}(j)} \diamond \tau_{\rho\left(f_{c}(j)\right)}^{(m)}
$$

where

$$
t_{f_{c}(j)}= \begin{cases}t_{c} & \text { if } f_{c}(\underline{k}) \notin F^{(1)} \\ t_{1} & \text { if } f_{c}(\underline{k}) \in F^{(1)}\end{cases}
$$

and

$$
\tau_{\rho\left(f_{c}(j)\right)}^{(m)}= \begin{cases}\tau_{j}^{(m)} & \text { if } f_{c}(\underline{k}) \notin F^{(1)} \\ \left(t_{1}\right)^{m} \diamond \tau_{\left(f_{c}(\underline{k}), f_{c} \circ U(\underline{k}), c\right)}^{(m-n)} & \text { if } f_{c}(\underline{k}) \in F^{(1)}\end{cases}
$$

The above shows that for $j^{\prime} \in \mathcal{J}^{(n)}$, we can find $\tau_{j^{\prime}}^{(m)}$ only by knowing the $\tau_{j}^{(m-n)}$ for $j \in \mathcal{J}$. In particular, for $j^{\prime} \in \mathcal{J}^{(2)}, \tau_{j^{\prime}}^{(m)}$ can be found when we know $\tau_{j}^{(m-1)}$ for $j \in \mathcal{J}$. But, we can also find the $\tau^{(m)}(j \in \mathcal{J})$ by knowing the $\tau_{j^{\prime}}^{(m)}\left(j^{\prime} \in \mathcal{J}^{(2)}\right)$, so we have obtained a recursive procedure for finding $\tau_{j}^{(m)}(j \in \mathcal{J})$ for all $m \in N$, given a starting distribution $\tau$. By formula 2.3, we are then also able to find $\tau_{j^{\prime}}^{(m)}\left(j^{\prime} \in \mathcal{J}^{(n)}\right)$ for all $m, n \in \mathbf{N}$ with $m \geq n$.

Now, let us consider the case where we have two different starting distributions, $\tau$ and $\theta$. Denote the corresponding distributions of the $\Delta \Theta($ etc. $)$ by $\tau_{j}^{(n)}$ and $\theta_{j}^{(n)}$, respectively, as in equation 2.3. Let $\vec{d}^{(n)}$ be the vector $\left(d\left(\tau_{j}^{(n)}, \theta_{j}^{(n)}\right)^{2}\right)_{j \in \mathcal{J}}$, i.e. a vector indexed by $\mathcal{J}$ with $d\left(\tau_{j}^{(n)}, \theta_{j}^{(n)}\right)^{2}$ as its $j^{\prime}$ th element.

An $n$-path is a sequence $\left\{j_{k}\right\}$ of elements of $\mathcal{J}^{(n)}$ such that if $j_{k}=(\underline{k}, \underline{l}, \vec{a})$ and $j_{k+1}=$ $(\underline{m}, \underline{n}, \vec{a})$, then $\underline{m}=\underline{l}$. The kind of paths we are interested in, are the 2-paths leading from one element of $F^{(1)}$ to a neighbour in $F^{(1)}$ via a 1-cell. The set of such paths will then be determined by a triplet in $\mathcal{J}$. For a $j \in \mathcal{J}$, let the set of paths be denoted $\Pi_{j}$, and let a single path be denoted just $\pi$.

Let us consider the case where we know $\vec{d}^{(n)}$ and $E\left[\tau_{j}^{(n)}\right]$ for all $j \in \mathcal{J}$. By applying 2.3 and then 2.1.2, we get for $j^{\prime}=f_{a}(\underline{i}, \underline{j}, b) \in \mathcal{J}^{(2)}$, that

$$
\begin{aligned}
d\left(\tau_{j^{\prime}}^{(n+1)}, \theta_{j^{\prime}}^{(n+1)}\right) & =d\left(t_{j^{\prime}} \diamond \tau_{\rho\left(j^{\prime}\right)}^{(n)}, t_{j^{\prime}} \diamond \theta_{\rho\left(j^{\prime}\right)}^{(n)}\right) \\
& =t_{j^{\prime}} \cdot d\left(\tau_{\rho\left(j^{\prime}\right)}^{(n)}, \theta_{\rho\left(j^{\prime}\right)}^{(n)}\right)
\end{aligned}
$$


Given a path $\pi \in \Pi_{j}$, let $\tau_{\pi}^{(n+1)}$ be the distribution of the time taken to traverse the path $\pi$ with transition times having distributions $\tau_{j}^{(n+1)}$, and define $\theta_{\pi}^{(n+1)}$ similarly. Then, by 2.1.3,

$$
\left(d\left(\tau_{\pi}^{(n+1)}, \theta_{\pi}^{(n+1)}\right)\right)^{2} \leq \sum_{k \in \pi}\left(t_{k}\right)^{2} \cdot\left[\left(d\left(\tau_{\rho(k)}^{(n)}, \theta_{\rho(k)}^{(n)}\right)\right)^{2}+\left(E\left[\tau_{\rho(k)}^{(n)}\right]-E\left[\theta_{\rho(k)}^{(n)}\right]\right)^{2}\right]
$$

where $t_{k}$ is defined in formula 2.4 .

Finally, letting $p_{\pi}$ be the probability that $\pi$ is the path in $\Pi$ that is chosen, we get

$$
\left(d\left(\tau_{j}^{(n+1)}, \theta_{j}^{(n+1)}\right)\right)^{2} \leq \sum_{\pi \in \Pi_{j}} p_{\pi} \cdot\left(d\left(\tau_{\pi}^{(n+1)}, \theta_{\pi}^{(n+1)}\right)\right)^{2}
$$

Putting together these equations, we get

$$
\left(d\left(\tau_{j}^{(n+1)}, \theta_{j}^{(n+1)}\right)\right)^{2} \leq \sum_{\pi \in \Pi_{j}} p_{\pi} \cdot \sum_{k \in \pi}\left(t_{k}\right)^{2} \cdot\left[d\left(\tau_{\rho(k)}^{(n)}, \theta_{\rho(k)}^{(n)}\right)^{2}+\left(E\left[\tau_{\rho(k)}^{(n)}\right]-E\left[\theta_{\rho(k)}^{(n)}\right]\right)^{2}\right]
$$

Let $\vec{x}^{(n)}=\left(E\left[\tau_{j}^{(n)}\right]\right)_{j \in \mathcal{J}}$, with expectation taken componentwise, and denote $\vec{x}^{(n)}$ 's $j$ 'th element $\vec{x}_{j}^{(n)}$. Via the same procedure as we used to find $\vec{d}^{(n)}$ from $\vec{d}^{(n+1)}$, we get that

$$
\vec{x}_{j}^{(n+1)}=\sum_{\pi \in \Pi_{j}} p_{\pi} \cdot \sum_{k \in \pi} t_{k} \cdot \vec{x}_{\rho(k)}^{(n+1)}
$$

Experienced from Th. 1.4.2, we immediately see that this gives us a matrix equation

$$
\vec{x}^{(n+1)}=\mathbf{M} \cdot \vec{x}^{(n)}
$$

But that means

$$
\vec{d}^{(n+1)} \leq \mathbf{M} \cdot \mathbf{D} \cdot \vec{d}^{(n)}
$$

where $\mathbf{D}_{i j}=\delta_{i j} \cdot t_{i}$, and $\mathbf{M}$ is the matrix from the equation above.

Now, $\mathbf{M}>0$, so we can use the theory of positive matrices, in particular the PerronFrobenius theory. It states that $\mathbf{M}$ has a positive real eigenvalue $r$ such that for any other eigenvalue $\lambda$ of $\mathbf{M},|\lambda| \leq r$, and that $r$ 's eigenvectors are unique up to multiplication by a constant. Moreover, the right and left eigenvectors belonging to $r$ are strictly positive .

For a vector $\vec{x}=\left(x_{1}, x_{2}, \ldots, x_{n}\right)$, we'll let $|\vec{x}|=\left(\left|x_{1}\right|,\left|x_{2}\right|, \ldots,\left|x_{n}\right|\right)$

\section{Lemma 2.2.2 $r=1$.}

Proof: Making an equation for the mean time to get from 1 to $\underline{0}$ and then back again to $\underline{1}$, and using from Theorem 1.4.2 that the mean time to get from $\underline{1}$ to $\underline{0}$ is 1 , we get

$$
\sum_{j \in \mathcal{J}} w_{j} \cdot x_{j}^{(n)}=2 \text { or, in vector notation: } \vec{w} \cdot \vec{x}^{(n)}=2
$$




\section{CHAPTER 2. BROWNIAN MOTION ON SIMPLE FRACTAL SPACES}

for each $n . \vec{w}$ is a vector independent of $\vec{x}^{(n)}$, whose $(\underline{k}, \underline{l}, a)$ 'th element is the average number of times $B^{(1)}$ crosses over from $\underline{k}$ to $\underline{l}$ via $C_{a}$. We see that $\vec{w}$ is componentwise larger than zero. We use $\vec{w}$ to make a norm by setting $\|\vec{x}\|_{\vec{w}}=\vec{w} \cdot|\vec{x}|$. We then have $\left\|\vec{x}^{(n)}\right\|_{\vec{w}}=2$ for all $n \in \mathbb{N}$.

If $\vec{z}$ is the (right) eigenvector belonging to $r$, we can multiply $\vec{z}$ by a constant $c_{1}$ so that $0<c_{1} \cdot \vec{z} \leq \vec{x}^{(1)}$. But then, for any $k \in N$, we get $0<\mathbf{M}^{k} \cdot\left(c_{1} \cdot \vec{z}\right) \leq \mathbf{M}^{k} \cdot \vec{x}^{(1)}=\vec{x}^{(k+1)}$, making $c_{1} \cdot\left\|\mathbb{M}^{k} \cdot \vec{z}\right\|_{\vec{w}}=c_{1} \cdot r^{k} \cdot\|\vec{z}\|_{\vec{w}} \leq 2$ for all $k \in \mathbb{N}$. This shows $r \leq 1$.

Similarly, there is a positive constant $c_{2}$ such that $0<c_{2} \cdot \vec{x}^{(1)} \leq \vec{z}$, so for any $k \in N$, $0<\mathbf{M}^{k} \cdot\left(c_{2} \cdot \vec{x}^{(1)}\right) \leq \mathbf{M}^{k} \cdot \vec{z}=r^{k} \cdot \vec{z}$, so $2 \cdot c_{2}<\left\|\mathbf{M}^{k} \cdot \vec{x}^{(1)}\right\|_{\vec{w}} \leq r^{k} \cdot\|\vec{z}\|_{\vec{w}}$ for all $k \in \mathbf{N}$. This shows $r \geq 1$.

Invoking [14, Theorem 1.2], we see that when we norm the right and left eigenvectors for the eigenvalue $r=1, \vec{l}$ and $\vec{r}$, by demanding $\vec{l} \cdot \vec{r}=1$, the matrix $\mathbf{A}=\vec{r} \circ \vec{l}$ has the property that $\left\|\mathbf{M}^{k}-\mathbf{A}\right\| \leq K_{1} \cdot \delta^{k}$, where $K_{1}>0$ and $\left|\lambda_{2}\right|<\delta<1$, where $\lambda_{2}$ is the eigenvalue of $\mathbf{M}$ that is the second largest in absolute value. We can take $\|\cdot\|$ as the operator norm.

Lemma 2.2.3 The vectors $\vec{x}^{(n)}$ converge to a limit $\vec{x}$ as $n \rightarrow \infty$ with geometric speed. That is, $\left\|\vec{x}^{(n)}-\vec{x}\right\| \leq K_{2} \cdot \delta^{n}, K_{2}>0$

\section{Proof:}

$$
\begin{aligned}
\lim _{n \rightarrow \infty} \vec{x}^{(n)} & =\lim _{n \rightarrow \infty} \mathbf{M}^{k} \cdot \vec{x}^{(1)} \\
& =\left(\lim _{n \rightarrow \infty} \mathbf{M}^{k}\right) \cdot \vec{x}^{(1)} \\
& =\mathbf{A} \cdot \vec{x}^{(1)}
\end{aligned}
$$

We call this limit $\vec{x}$. Applying any norm $\|\cdot\|$ (all norms on $\mathbb{R}^{n}$ are equivalent),

$$
\begin{aligned}
\left\|\vec{x}^{(k)}-\vec{x}\right\| & =\left\|\mathbf{M}^{k-1} \cdot \vec{x}^{(1)}-\mathbf{A} \cdot \vec{x}^{(1)}\right\| \\
& =\left\|\left(\mathbf{M}^{k-1}-\mathbf{A}\right) \cdot \vec{x}^{(1)}\right\| \\
& \leq\left\|\left(\mathbf{M}^{k-1}-\mathbf{A}\right)\right\| \cdot\left\|\vec{x}^{(1)}\right\| \\
& \leq\left\|\vec{x}^{(1)}\right\| \cdot K_{1} \cdot \delta^{k-1}
\end{aligned}
$$

We let $\vec{\tau}^{(n)}=\left(\tau_{j}^{(n)}\right)_{j \in \mathcal{J}^{\prime}}$, and let $d\left(\vec{\tau}^{(n)}, \vec{\theta}^{(n)}\right)=\left(\sum_{j \in \mathcal{J}} d\left(\vec{\tau}^{(n)}, \vec{\theta}^{(n)}\right)^{4}\right)^{\frac{1}{4}}=\sqrt{\left\|\vec{d}^{(n)}\right\|}$, with \|. $\|$ Euclidean norm.

For the following proposition we need to define another operation denoted by $\diamond$, multiplication of vectors element by element, i.e. $\left(a_{1}, a_{2}, \ldots, a_{n}\right) \diamond\left(b_{1}, b_{2}, \ldots, b_{n}\right)=\left(a_{1} b_{1}, a_{2} b_{2}, \ldots, a_{n} b_{n}\right)$ $(\vec{a})^{\diamond 2}=\vec{a} \diamond \vec{a}$. 
Proposition 2.2.4 There exists a vector of distributions indexed by $\mathcal{J}$ which is the limit of the distribution vectors $\vec{\tau}^{(n)}$, and which is independent of which initial distribution $\tau \in \mathcal{G}$ with $E[\tau]=1$ we choose.

Proof: Independence: Take the two initial distributions, $\tau$ and $\theta$. We have $E[\tau]=$ $E[\theta]=1$. That implies $E\left[\tau_{j}^{(n)}\right]=E\left[\theta_{j}^{(n)}\right]$ for all $n \in N$ and all $j \in \mathcal{J}$. Thus formula 2.5 in this case modifies to, in vector form

$$
\vec{d}^{(n+1)} \leq \mathbf{M} \cdot \mathbf{D} \cdot \vec{d}^{(n)}
$$

Since $\left\|\mathrm{M}^{n}\right\| \leq\|\mathbf{A}\|+K_{1}$ for all $n \in \mathbb{N},\|\mathbf{D}\|=t_{\max _{1}}<1$, and $\vec{d}^{(n)} \geq 0$, we have

$$
\begin{aligned}
\left\|\vec{d}^{(n+1)}\right\| & \leq\left\|(\mathbf{M} \cdot \mathbf{D})^{n} \cdot \vec{d}^{(1)}\right\| \\
& \leq\left\|(\mathbf{M} \cdot \mathbf{D})^{n}\right\| \cdot\left\|\vec{d}^{(1)}\right\| \\
& \leq\left\|\mathbf{M}^{n}\right\| \cdot\left\|\mathbf{D}^{n}\right\| \cdot\left\|\vec{d}^{(1)}\right\| \\
& \leq\left(\|\mathbf{A}\|+K_{1}\right) \cdot\left(t_{\max _{1}}\right)^{n} \cdot\left\|\vec{d}^{(1)}\right\| \stackrel{n \rightarrow \infty}{\longrightarrow} 0
\end{aligned}
$$

We have shown that the limit is independent of the choice of starting distribution, if the limit exists.

Existence: Now, if we pick an arbitrary starting distribution, $\tau$, that induces one particular $\vec{\tau}^{(1)}$, we can define $\vec{\theta}$ by letting $\vec{\theta}^{(1)}=\vec{\tau}^{(2)}$. For the $\vec{d}^{(n)}$ given by these $\vec{\tau}^{(n)}$ and $\vec{\theta}^{(n)}$, we get

$$
\begin{aligned}
\vec{d}^{(n+1)} & \leq \mathbf{M} \cdot \mathbf{D} \cdot\left(\vec{d}^{(n)}+\left(\vec{x}^{(n)}-\vec{x}^{(n-1)}\right)^{\diamond 2}\right) \\
& \leq(\mathbf{M} \cdot \mathbf{D})^{n} \cdot \vec{d}^{(1)}+\sum_{k=1}^{n}\left((\mathbf{M} \cdot \mathbf{D})^{n-k}\left(\vec{x}^{(k)}-\vec{x}^{(k-1)}\right)^{\diamond 2}\right)
\end{aligned}
$$

And so,

$$
\begin{aligned}
\left\|\vec{d}^{(n+1)}\right\| & \leq\left\|(\mathbf{M} \cdot \mathbf{D})^{n}\right\| \cdot\left\|\vec{d}^{(1)}\right\|+\sum_{k=1}^{n}\left\|(\mathbf{M} \cdot \mathbf{D})^{n-k}\right\| \cdot\left\|\left(\vec{x}^{(k)}-\vec{x}^{(k-1)}\right)^{\diamond 2}\right\| \\
& \leq\left\|\mathbf{M}^{n}\right\| \cdot\left\|\mathbf{D}^{n}\right\| \cdot\left\|\vec{d}^{(1)}\right\|+\sum_{k=1}^{n}\left\|\mathbf{M}^{n-k}\right\| \cdot\left\|\mathbf{D}^{n-k}\right\| \cdot\left\|\left(\vec{x}^{(k)}-\vec{x}^{(k-1)}\right)\right\|^{2} \\
& \left.\leq\left(\|\mathbf{A}\|+K_{1}\right) \cdot\left(t_{\max _{1}}\right)^{n} \cdot\left\|\vec{d}^{(1)}\right\|+\sum_{k=1}^{n}\left(\|\mathbf{A}\|+K_{1}\right) \cdot\left(t_{\max _{1}}\right)^{n-k} \cdot\left(2 K_{2} \cdot \delta^{k}\right)^{2}\right) \\
& \leq\left(\|\mathbf{A}\|+K_{1}\right) \cdot\left(\left\|\vec{d}^{(1)}\right\|+4\left(K_{2}\right)^{2} \cdot n\right) \cdot\left(\delta^{2} \vee t_{\max _{1}}\right)^{n}
\end{aligned}
$$

Now, since $d\left(\vec{\tau}^{(n)}, \vec{\tau}^{(n+1)}\right)=d\left(\vec{\tau}^{(n)}, \vec{\theta}^{(n)}\right)=\sqrt{\left\|\vec{d}^{(n)}\right\|}$, we see that the sequence of vectors of distributions, $\left\{\bar{\tau}^{(n)}\right\}_{n \in \mathbb{N}}$, is Cauchy, and by the completeness of $(\mathcal{G}, d)$, it has a limit.

Let $\vec{\tau}=\left(\tau_{j}\right)_{j \in \mathcal{J}}$ be this limit. By using formula 2.3 we find the distribution functions for the transition times on $F^{(n)}$ for all the $n>1$. We then get our decimation-invariant random walks. 


\subsection{Decimation Invariant Random walks}

I will now construct the random walks that are the limit of $W_{m}^{(n)}$ as $m$ tends to infinity, $W^{(n)}$, on $F^{(n)}$, by letting $W^{(n)}$ be the random walk with the transition probabilities of $B^{(n)}$, and time distributions given by the fixpoint $\tau=\left(\tau_{j}\right)_{j \in \mathcal{J}}$ and the limit version of 2.3 , which simply is

$$
\tau_{f_{c}(j)}=t_{f_{c}(j)} \diamond \tau_{\rho\left(f_{c}(j)\right)}
$$

Let $\Theta_{k}^{(n)}$ be an increasing sequence of random variables for each $n$ with

1. $\Theta_{0}^{(n)}=0$

2. If $\Delta \Theta_{k}^{(n)}=\Theta_{k}^{(n)}-\Theta_{k-1}^{(n)}$, then the distribution of $\left.\Delta \Theta_{k}^{(n)}\right|_{B^{(n)}(k-1)=\underline{i}, B^{(n)}(k)=\underline{j}, C^{(n)}(k)=C_{d}}$ is $\tau_{(\underline{i}, \underline{j}, \vec{a})}$.

3. The $\left.\Delta \Theta_{k}^{(n)}\right|_{B^{(n)}(k-1)=\underline{i}, B^{(n)}(k)=\underline{j}, C^{(n)}(k)=C_{a}}$ are independent of each other and each of them conditionally independent of $\left.C^{(n)}(k)\right|_{B^{(n)}(k-1)}$.

We define our random walks as

$$
W^{(n)}(t, \omega)=B^{(n)}(k, \omega), \text { where } k=\max \left\{i: \Theta_{i}^{(n)}(\omega) \leq t\right\}
$$

The $W^{(n)}$ are decimation invariant, in the sense that if $n<m$ and $W^{(m)}$ is started at a point in $F^{(n)}$, then $\left.W^{(m)}\right|_{F^{(n)}}$ has the same law as $W^{(n)}$. For the next proposition, let $\Theta(\omega)=\min \left\{t>0: W^{(1)}\left(t+t^{\prime}, \omega\right)=\underline{1}\right.$, where $\left.W^{(1)}\left(t^{\prime}, \omega\right)=\underline{0}\right\}$, and let $\tau=\sigma_{\Theta}$.

Proposition 2.3.1 Let $q$ be the minimal number of cells connecting $\underline{1}$ to $\underline{0}$, and let $\beta=-\frac{\log q}{\log t_{\min _{1}}}$. Then there exists a constant $K>1$ such that

$$
\tau_{j}([0, t]) \leq K^{-t^{-\beta}} \forall j \in \mathcal{J}
$$

for all $t \in(0,1]$

Proof: Given an $a \in(0,1]$, we can find a $K>1$ such that 2.6 holds for any $t \in[a, 1]$. Just let $K=\min \left\{\left(\tau_{j}([0, t])\right)^{-t^{\beta}}: t \in[a, 1] \wedge j \in \mathcal{J}\right\}$. We choose $a=t_{\min _{1}}$.

Assume there is a smallest number $s$ in $(0,1]$ such that equation 2.6 holds for all $t \in[s, 1]$. If I can prove that 2.6 holds for all $t \in\left[t_{\min _{1}} \cdot s, s\right)$, I have a reductio ad absurdum on the assumption, and the lemma is proved. 
By definition of $K$ and $a,\left[s, \frac{s}{a}\right] \subseteq[s, 1]$. But also, if $j^{\prime} \in \mathcal{J}^{(2)}$, then $\tau_{j^{\prime}}=t_{a} \diamond \tau_{j}$ for some $a \in A$ and $j \in \mathcal{J}$. So if $t \in\left[t_{\min _{1}} \cdot s, s\right)$, we have uniformly for $j^{\prime} \in \mathcal{J}^{(2)}$

$$
\begin{aligned}
\tau_{j^{\prime}}([0, t]) & =\left(t_{a} \diamond \tau_{j}\right)([0, t]) \\
& =\tau_{j}\left(\left[0, \frac{t}{t_{a}}\right]\right) \\
& \leq \tau_{j}\left(\left[0, \frac{t}{t_{\min _{1}}}\right]\right) \\
& \leq K^{-\left(\frac{t}{t_{\min _{1}}}\right)^{-\beta}}
\end{aligned}
$$

Now, the probability $W^{(2)}$ has crossed a 1 -cell in the interval $[0, t]$ is less than or equal to the probability that the process has crossed the $q 2$-cells required to do that, or even crossed any $q$ 2-cells at all. In particular, for our chosen $t$,

$$
\begin{aligned}
\tau_{j}([0, t]) & \leq \prod_{i=1}^{q} \tau_{j_{q}^{\prime}}([0, t]) \\
& \leq \prod_{i=1}^{q} K^{-\left(\frac{t}{t_{\min _{1}}}\right)^{-\beta}} \\
& =\left(K^{-\left(\frac{t}{t_{\min _{1}}}\right)^{-\beta}}\right)^{q} \\
& =K^{-t^{-\beta}}
\end{aligned}
$$

where $j_{q}^{\prime}$ indexes the 2-cells crossed by $W^{(2)}$.

\subsection{Brownian motion}

I will in the rest of the paper make much use of non-standard analysis, as I find this to be the best tool for handling the kind of problems that arise here.

Proposition 2.4.1 For all $N \in^{*} N-N, W^{N}(t, \omega)$ is $S$-continuous for $L\left({ }^{*} P\right)$-almost all $\omega \in \Omega$. Actually, there exists a subset $\Omega^{\prime} \subset \Omega$ with $L\left({ }^{*} P\right)$-measure 1 such that for all $\omega \in \Omega^{\prime}$ there exists a constant $C_{\omega}$ such that

$$
d\left(W^{N}(t, \omega), W^{N}(s, \omega)\right)^{T} \leq C_{\omega}|t-s|\left(\log \frac{1}{|t-s|}\right)^{\frac{1}{\beta}}
$$

for all $s, t \in^{*}[0,1], s \not t$, where $T$ is the time scaling exponent from Section 1.4, and $\beta$ is the $\beta$ from proposition 2.3.1.

Proof: Let $W^{N}(t, \omega)$ be written $X(t, \omega)$. We also have need of a function

$$
f(r)=|r|^{\frac{1}{T}}\left(\log \frac{1}{|r|}\right)^{\frac{1}{\beta T}}
$$




\section{CHAPTER 2. BROWNIAN MOTION ON SIMPLE FRACTAL SPACES}

Pick two arbitrary points $s, t \in^{*}[0,1], s<t, s \not t$. We see that if there are two values $u, v \in^{*}[s, t]$ such that $d(X(u), X(v)) \geq 2 r$ for some positive number $r$, then $X$ must have crossed from $\underline{i}$ to $\underline{j} \in F^{(n)}$ for some $n \in N$ via some $n$-cell $C_{\vec{a}}$ with $r_{\vec{a}} \geq r \cdot r_{\min _{1}}$ during the interval $*[u, v]$. Applying (the *-transfer of) proposition 2.3.1 to this case, with $j^{\prime}=(\underline{i}, \underline{j}, \vec{a})$, we get, using an appropriate $j \in \mathcal{J}$, that

$$
\begin{aligned}
{ }^{*} P\left\{\exists u, v \in^{*}[s, t]: d(X(u), X(v)) \geq 2 r\right\} & \leq \sup _{u, v \in^{*}[s, t]}{ }^{*} \tau_{\vec{a}}\left({ }^{*}[0, u-v]\right) \\
& \leq \sup _{u, v \in^{*}[s, t]}\left(t_{\vec{a}} \diamond^{*} \tau_{j}\right)\left({ }^{*}[0, u-v]\right) \\
& =\sup _{u, v \in^{*}[s, t]}{ }^{*} \tau_{j}\left({ }^{*}\left[0, \frac{u-v}{t_{\vec{a}}}\right]\right) \\
& \leq{ }^{*} \tau_{j}\left({ }^{*}\left[0, \frac{t-s}{t_{\vec{a}}}\right]\right) \\
& \leq K^{-\left(\frac{t-s}{t_{\vec{d}}}\right)^{-\beta}}
\end{aligned}
$$

Let $C$ be some constant greater than $(\log K)^{-\frac{1}{\beta T}}$, and pick $r$ so small that $C f(t-s) \geq 2 r$. Then the above implies that

$$
{ }^{*} P\left\{\exists u, v \in^{*}[s, t]: d(X(u), X(v)) \geq C f(t-s)\right\} \leq K^{-\left(\frac{t-s}{t_{d}}\right)^{-\beta}}
$$

But

$$
\begin{aligned}
\left(t_{\vec{a}}\right)^{-\beta} & =\left(r_{\vec{a}}\right)^{-\beta T} \\
& \leq\left(\frac{C}{2} f(t-s)\right)^{-\beta T} \\
& =C^{\prime}|t-s|^{\beta} \log \frac{1}{|t-s|} \cdot(\log K)^{-1}
\end{aligned}
$$

where $C^{\prime}=\left(\frac{C}{2}\right)^{\beta T} \log K$, so we have the general relation

$$
{ }^{*} P\left\{\exists u, v \in^{*}[s, t]: d(X(t), X(s)) \geq C f(t-s)\right\} \leq|t-s|^{C^{\prime}}
$$

In particular,

$$
{ }^{*} P\left\{\exists u, v \in^{*}\left[\frac{k}{2^{n}}, \frac{k+1}{2^{n}}\right]: d(X(t), X(s)) \geq C_{1} f\left(2^{-n}\right)\right\} \leq 2^{-n C^{\prime}}
$$

when $k<2^{n}$, for all $n \in I$, where $I \subset^{*} \mathrm{~N}$ is internal, and $I \supset \mathrm{N}$. That is, there exists an $H \in^{*} \mathbf{N}-\mathrm{N}$ such that the above formula is true for all $n \leq H$. Pick one $m \leq H$. Then

$$
\begin{aligned}
& { }^{*} P\left(\left\{\exists n(m \leq n \leq H) \exists k<2^{n} \exists u, v \in^{*}\left[\frac{k}{2^{n}}, \frac{k+1}{2^{n}}\right]: d(X(t), X(s)) \geq C f\left(2^{-n}\right)\right\}\right) \\
& \leq \sum_{n=m}^{H} \sum_{k<2^{n}}^{*} P\left(\left\{\exists u, v \in^{*}\left[\frac{k}{2^{n}}, \frac{k+1}{2^{n}}\right]: d(X(t), X(s)) \geq C f\left(2^{-n}\right)\right\}\right) \\
& \leq \sum_{n=m}^{H} \sum_{k<2^{n}} 2^{-n C^{\prime}}=\sum_{n=m}^{H}\left(2^{1-C^{\prime}}\right)^{n} \leq \frac{2^{-m\left(1-C^{\prime}\right)}}{1-2^{1-C^{\prime}}}<\infty
\end{aligned}
$$


Let $\Omega_{m}=\left\{\omega \in \Omega: \forall n(m \leq n \leq H) \forall k<2^{n} \forall u, v \in^{*}\left[\frac{k}{2^{n}}, \frac{k+1}{2^{n}}\right]\left(d(X(u), X(v))<C f\left(2^{-n}\right)\right)\right\}$. $\left\{\Omega_{m}\right\}$ is an increasing sequence of sets, with ${ }^{*} P\left\{\Omega_{m}\right\}=1-\frac{2^{-m\left(1-C^{\prime}\right)}}{1-2^{1-C^{\prime}}}$.

Changing into Loeb theory, we can define $\Omega^{\prime}=\bigcup_{m \in \mathbb{N}} \Omega_{m} . L\left({ }^{*} P\right)\left(\Omega^{\prime}\right)=1$. We shall write $\bar{P}$ for $L\left({ }^{*} P\right)$. Then, for all $\omega \in \Omega^{\prime}$, there exists an $n(\omega) \in N$ such that

$$
d(X(u), X(v))<C_{1} f\left(2^{-n}\right) \quad \forall u, v \in^{*}\left[\frac{k}{2^{n}}, \frac{k+1}{2^{n}}\right] \forall k<2^{n} \forall n \geq n(\omega)
$$

Now fix $s, t$, two arbitrary numbers in ${ }^{*}[0,1], s \not t$, and let $s<t$. Let $p=n(\omega) \vee$ $-\left[\left[\frac{\log |t-s|}{\log 2}\right]\right]$, where $[[x]]$ gives the largest integer smaller than $x$. The interval ${ }^{*} t[s, t]$ is intersected by at most $3 \vee 2^{n(\omega)}$ intervals of the kind ${ }^{*}\left[\frac{k}{2^{p}}, \frac{k+1}{2^{p}}\right], k<2^{p}$. Index these intervals by $\mathcal{I}$, naming the $i$ th interval $I_{i}$. We then have our relation

$$
\begin{aligned}
d(X(s), X(t)) & \leq \sup \left\{d(X(u), X(v)): u, v \in^{*}[s, t]\right\} \\
& \leq \sum_{i \in \mathcal{I}} \sup \left\{d(X(u), X(v)): u, v \in I_{j}\right\} \\
& \leq \sum_{j \in J} C f\left(2^{-p}\right)=\left(3 \vee 2^{n(\omega)}\right) C f\left(2^{-p}\right) \\
& \leq\left(3 \vee 2^{n(\omega)}\right) C f(t-s)
\end{aligned}
$$

We get our Brownian motion simply by putting $B(t, \omega)={ }^{\circ} W^{N}(t, \omega)$, for some $N \epsilon^{*}$ $N-N$.

Corollary 2.4.2 The Brownian motion is continuous for $L\left({ }^{*} P\right)$-almost all $\omega \in \Omega$. Actually, there exists a subset $\Omega^{\prime} \subset \Omega$ with $L\left({ }^{*} P\right)$-measure 1 such that for all $\omega \in \Omega^{\prime}$ there exists a constant $C_{\omega}$ such that

$$
d(B(t), B(s))^{T}=C_{\omega}|t-s|\left(\log \frac{1}{|t-s|}\right)^{\frac{1}{\beta}}
$$

for all $s, t \in[0,1]$. 


\section{Chapter 3}

\section{The Markov Property}

Our process $W^{(N)}$ is clearly not Markov if the cells are of different sizes, as the probability of which site the particle will go to next after it has arrived at one site, is different than the probability of which site it will choose after having waited for some time. Still, we expect the standard part process to be Markov, as the dependency described above is a.s. confined to the infinitesimal level.

\subsection{Some properties of the process}

Lemma 3.1.1 If $f \in C(\mathbf{K}, \mathbf{R})$, then $E^{x}\left[{ }^{*} f\left(W^{(N)}\right)(\omega, t)\right] \simeq E^{y}\left[{ }^{*} f\left(W^{(N)}\right)(\omega, t)\right]$ whenever $x \simeq y, N \in{ }^{*} \mathrm{~N}-\mathrm{N}$, and $t \in^{*}[0, \infty)$.

Proof: We can without loss of generality assume $x$ and $y$ are contained in the same $N$-cell, for some $N \in^{*} \mathbf{N}-\mathrm{N}$, since otherwise we could pick a third element $z$ such that the assumption is true for both the pair $x, z$ and the pair $y, z$, whereupon the lemma holds by the transitivity of $\simeq$.

Pick an $M \in^{*} N-N$ and an $\vec{a} \in A^{M}$ such that $x, y \in C_{\vec{a}}$ and $\phi_{\vec{a}}^{-1}(x) \simeq \phi_{\vec{a}}^{-1}(y)$. Fixing $v=\phi_{\vec{a}}(\underline{0}), w=\phi_{\vec{a}}(\underline{1}), x^{\prime}=\phi_{\vec{a}}^{-1}(x)$ and $y^{\prime}=\phi_{\vec{a}}^{-1}(y)$, we get

$$
\begin{gathered}
P^{x}\left[W^{(N)}(\omega, \sigma)=v\right]=P^{x^{\prime}}\left[W^{(N-M)}\left(\omega^{\prime}, \sigma^{\prime}\right)=\underline{0}\right] \begin{array}{r}
=v_{x^{\prime}} \\
\text { and }
\end{array} \\
P^{y}\left[W^{(N)}(\omega, \sigma)=v\right]=P^{y^{\prime}}\left[W^{(N-M)}\left(\omega^{\prime}, \sigma^{\prime}\right)=\underline{0}\right]=v_{y^{\prime}}
\end{gathered}
$$

so the following equation holds

$$
\begin{aligned}
E^{x}\left[{ }^{*} f\left(W^{(N)}\right)(\omega, t+\sigma)\right] & \simeq v_{x^{\prime}} \cdot E^{v}\left[{ }^{*} f\left(W^{(N)}\right)(\omega, t)\right]+\left(1-v_{x^{\prime}}\right) \cdot E^{\omega}\left[{ }^{*} f\left(W^{(N)}\right)(\omega, t)\right] \\
& \simeq v_{y^{\prime}} \cdot E^{v}\left[{ }^{*} f\left(W^{(N)}\right)(\omega, t)\right]+\left(1-v_{y^{\prime}}\right) \cdot E^{\omega}\left[{ }^{*} f\left(W^{(N)}\right)(\omega, t)\right] \\
& \simeq E^{y}\left[{ }^{*} f\left(W^{(N)}\right)(\omega, t+\sigma)\right]
\end{aligned}
$$


First and last $\simeq$ by the a.s. S-continuity of $W^{(N)}$, and middle $\simeq$ by that $x^{\prime} \simeq y^{\prime}$ and 1.3.8.

\subsection{A random walk with the Markov property}

We will have to construct our 4 th random walk process, $Z^{(n)}$, in order to prove the Markov property. First, I will show that it for $N$ infinite, $Z^{(N)}$ has a standard part that is a.s. equal to our decimation invariant random walks from the previous chapter. Then I will show that $Z^{(n)}$, and the standard part of $Z^{(N)}$, are [strong] Markov. Since now $Z^{(N)}$ 's standard part is strong Markov and a.s. equal to the standard parts of the previous preocesses, their standard parts will be strong Markov, too.

The problem with the previous random walks was that if a particle had arrived at a site, and stayed there for some amount of time, the distribution of which site to go to next, changed with the time the particle has waited. So, to mend this, $Z^{(n)}$ is defined on a discrete time line, where the particle after one time unit at a "proper" site jumps to a waiting site in which it waits for a jump to a unique "proper" site. At the waiting site, the probability of leaving the waiting site be equally large at all points of time, with the leaving probability calibrated so that the mean transition time over a cell $C_{\vec{a}}$ is $t_{\vec{a}}$. In mathematical writing, the process is given thus:

Let $K=\left[\left[\left(t_{\min _{1}}\right)^{-n}\right]\right]$, and then let $\Delta t=\frac{1}{K !}$. We then define the discrete time line $T_{(n)}=\left\{n \cdot \Delta t \mid n \in^{*} N_{0}\right\}$. Now, letting $\iota \in \mathbb{K}$ be chosen so that $0<d(\underline{0}, \iota)<\frac{1}{2}$, define for every $\vec{a} \in A^{*}$,

$$
\psi_{\vec{a}}\left(\phi_{\vec{a}}(\underline{0})\right)=\phi_{\vec{a}}(\iota) \text { and } \psi_{\vec{a}}\left(\phi_{\vec{a}}(\underline{1})\right)=\phi_{\vec{a}} \circ U(\iota)
$$

and let $E^{(n)}=\bigcup_{\vec{a} \in A^{n}} \bigcup_{\substack{x \in F^{(n)} \\ x \in C_{\vec{d}}}} \psi_{\vec{a}}(x)$

Let $Z^{(n)}: \Omega \times T_{(n)} \rightarrow F^{(n)} \cup E^{(n)}$ be our process, written $z_{t}$ for short, and define it recursively, for all $\vec{a}$ in $A^{n}$ :

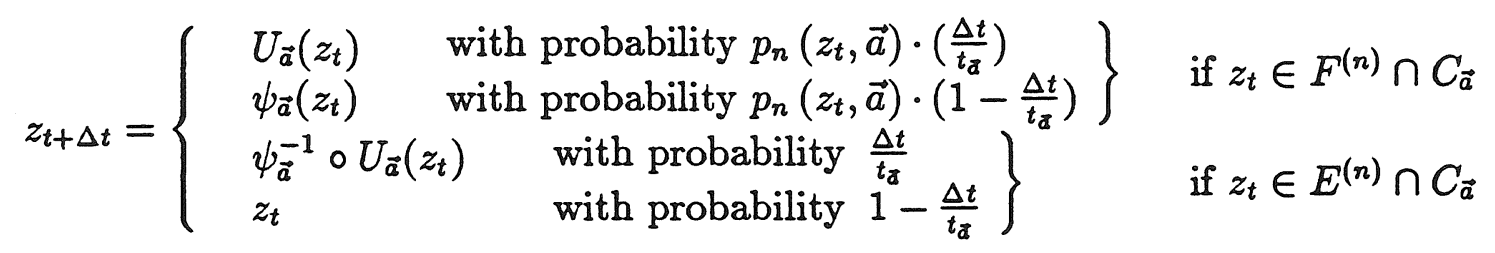

where $U_{\vec{a}}=\phi_{\vec{a}} \circ U \circ \phi_{\vec{a}}^{-1}$. All the usual independencies are assumed. Then $Z^{(n)}$ is Markov by definition.

We will now show that for $N$ infinite, $Z^{(N)}$ is equivalent to the decimation invariant walk $W^{(N)}$ from section 2.3. This will be by showing them both to be equal to the initial process $X^{(N)}$ from section 2.2, and thereupon using transitivity: 
Lemma 3.2.1 For $N \in{ }^{*} N-N, W^{(N)}(\omega, t) \simeq Z^{(N)}(\omega, t)$ for all $t \in \cup_{m \in N^{*}}[0, m] \cap T_{(n)}$, for almost all $\omega \in \Omega$.

Proof: We will do this proof in two steps. First, we show that $W^{(N)}(\omega, t) \simeq X^{(N)}(\omega, t)$ for almost all $\omega \in \Omega$, and then that $X^{(N)}(\omega, t) \simeq Z^{(N)}(\omega, t)$ for almost all $\omega \in \Omega$. The lemma then follows by the transitivity of $\simeq$.

$\underline{W^{(N)}(\omega, t) \simeq X^{(N)}(\omega, t)}:$ Pick $M, H \in^{*} \mathrm{~N}-\mathrm{N}$ such that $M+H=N$. Then

$$
\vec{\tau}^{(H)} \simeq^{*} \vec{\tau}
$$

If we consider $X^{(N)}$ and $W^{(N)}$ restricted to $F^{(M)}$, they become $W_{N}^{(M)}$ and $W^{(M)}$, respectively. The time required for the processes, started at some point in $F^{(N)}$, to hit $F^{(M)}$ for the first time, is infinitesimal a.s.. Since also $W^{(N)}$ is S-continuous a.s., it suffices to show that $\left.\left.W^{(N)}(\omega, t)\right|_{F^{(M)}} \simeq X^{(N)}(\omega, t)\right|_{F^{(M)}}$, i.e. that $W^{(M)}(\omega, t) \simeq W_{N}^{(M)}(\omega, t)$, for almost all $\omega \in \Omega$. We couple the processes by requiring that they are defined from the same simple Markov process, $B^{(M)}$, but let their transition times, given the paths, be independent. For simplicity of notation, write $\Gamma$ for $W_{N}^{(M)}$, and $\Theta$ for $\Theta^{(M)}$.

Let $K=\max _{j \in \mathcal{J}} \frac{\left\|\tau_{j}\right\|^{2}}{\left(E\left[\tau_{j}\right]\right)^{2}}$. Then, since $\sigma_{\Delta \Theta_{k}}$ is a scaled version of a $\tau_{j}$ for some $j \in \mathcal{J}$, that is, $\sigma_{\Delta \Theta_{k}}=\tilde{t} \diamond \tau_{j}$ for some $\tilde{t} \in(0,1)$, we get $E\left[\left(\Delta \Theta_{k}\right)^{2}\right] \leq K \cdot\left(E\left[\Delta \Theta_{k}\right]\right)^{2}$

What 3.1 says, is that $\frac{E\left[\left(\Delta \Theta_{k}\right)^{2}\right]}{E\left[\left(\Delta \Gamma_{k}\right)^{2}\right]} \simeq 1$ and $\frac{E\left[\Delta \Theta_{k}\right]}{E\left[\Delta \Gamma_{k}\right]} \simeq 1$. So, if $K^{\prime}$ is a real number slightly larger than $K$, then $E\left[\left(\Delta \Theta_{k}\right)^{2}\right] \leq\left(E\left[\Delta \Theta_{k}\right]\right)^{2} \cdot K^{\prime}$, and $E\left[\Delta \Theta_{k}-\Delta \Gamma_{k}\right]=\partial \cdot E\left[\Delta \Theta_{k}\right]$, where $\partial \simeq 0$.

Using this, we get

$$
\begin{aligned}
E\left[\left(\Theta_{k}-\Gamma_{k}\right)^{2}\right] & =E\left[\left(\sum_{l=1}^{k}\left(\Delta \Theta_{l}-\Delta \Gamma_{l}\right)\right)^{2}\right] \\
& =E\left[\sum_{l=1}^{k}\left(\Delta \Theta_{l}-\Delta \Gamma_{l}\right)^{2}+\sum_{l=1}^{k} \sum_{m>l}\left(\Delta \Theta_{l}-\Delta \Gamma_{l}\right) \cdot\left(\Delta \Theta_{m}-\Delta \Gamma_{m}\right)\right] \\
& =\sum_{l=1}^{k} E\left[\left(\Delta \Theta_{l}-\Delta \Gamma_{l}\right)^{2}\right]+\sum_{l=1}^{k} \sum_{m>l} E\left[\Delta \Theta_{l}-\Delta \Gamma_{l}\right] \cdot E\left[\Delta \Theta_{m}-\Delta \Gamma_{m}\right] \\
& \leq \sum_{l=1}^{k} E\left[\left(\Delta \Theta_{l}\right)^{2}\right]+E\left[\left(\Delta \Gamma_{l}\right)^{2}\right]+\sum_{l=1}^{k}\left(\left(\partial \cdot E\left[\Delta \Theta_{l}\right]\right) \cdot \sum_{m>l}\left(\partial \cdot E\left[\Delta \Theta_{l}\right]\right)\right) \\
& \leq \sum_{l=1}^{k}\left(E\left[\Delta \Theta_{k}\right]\right)^{2} \cdot\left(K+K^{\prime}\right)+\left(\partial \cdot \sum_{l=1}^{k} E\left[\Delta \Theta_{k}\right]\right)^{2} \\
& \leq\left(\sum_{l=1}^{k} E\left[\Delta \Theta_{k}\right]\right) \cdot\left(t_{\max _{1}}\right)^{M} \cdot\left(K+K^{\prime}\right)+\partial^{2} \cdot\left(E\left[\Theta_{k}\right]\right)^{2} \\
& \leq E\left[\Theta_{k}\right] \cdot\left(t_{\max _{1}}\right)^{M} \cdot\left(K+K^{\prime}\right)+\partial^{2} \cdot\left(E\left[\Theta_{k}\right]\right)^{2} \simeq 0
\end{aligned}
$$




\section{CHAPTER 3. THE MARKOV PROPERTY}

for $\mathrm{k}$ so small that $E\left[\Theta_{k}\right]$ is finite

Thus $\Theta_{k} \simeq \Gamma_{k}$ a.s. for $\mathrm{k}$ so small that $E\left[\Theta_{k}\right]$ is finite. So, if $k(t)=\max \left\{k \mid \Gamma_{k} \leq t\right\}$,

$$
\begin{aligned}
W_{N}^{(M)}(\omega, t) & \simeq W_{N}^{(M)}(\omega, t) \\
& =B^{(M)}(\omega, k(t)) \\
& =W^{(M)}\left(\omega, \Theta_{k(t)} t\right) \\
& \simeq W^{(M)}(\omega, t)
\end{aligned}
$$

The last $\simeq$ by 2.4 .1 .

Thus 2.4.1 applies to $W_{N}^{(M)}$, and thereby to $X^{(N)}$, too.

$\underline{X^{(N)}(\omega, t) \simeq Z^{(N)}(\omega, t)}$ : Let $Y^{(n)}=\left.Z^{(n)}\right|_{F^{(n)}}$, and couple $Y^{(n)}$ with $X^{(n)}$ by requiring that they are defined from the same $B^{(n)}$, but let their transition times, given the paths, be independent.

Define $\Upsilon_{k+1}^{(n)}(\omega)=\min \left\{t \in^{*} \mathbf{R}_{0}^{+} \mid t>\Upsilon_{k}^{(n)}(\omega) \wedge Y^{(n)}(\omega, t) \in F^{(n)}-Y^{(n)}\left(\omega, \Upsilon_{k}^{(n)}(\omega)\right)\right\}$, and let $\Upsilon_{0}^{(n)} \doteq 0$.

So, let $N \in^{*} N-N$, and let $\Upsilon=\Upsilon^{(N)}$ and $T=T^{(N)}$. What we need to show, is that $\Upsilon_{k}(\omega) \simeq T_{k}(\omega)$ for almost all $\omega$. To do this, we first need to compute the $E\left[\left(\Delta \Upsilon_{k}\right)^{2}\right]$ : Consider the case that $Y^{(N)}$ 's $k^{\prime}$ 'th step be from the point $x$ over the cell $C_{\vec{a}}$. We see from the definition that

$$
\begin{aligned}
E\left[\left(\Delta \Upsilon_{k}\right)^{2}\right] & =(\Delta t)^{2} \cdot\left(1^{2}+\left(2^{2}-1^{2}\right) \cdot\left(1-\frac{\Delta t}{t_{\vec{a}}}\right)+\cdots+\left((n+1)^{2}-n^{2}\right) \cdot\left(1-\frac{\Delta t}{t_{\vec{a}}}\right)^{n}+\cdots\right. \\
& =(\Delta t)^{2} \cdot \sum_{n=0}^{\infty}(2 n+1) \cdot\left(1-\frac{\Delta t}{t_{\vec{a}}}\right)^{n} \\
& =2 \cdot\left(t_{\vec{a}}\right)^{2}-(\Delta t) \cdot t_{\vec{a}} \leq 2 \cdot\left(t_{\vec{a}}\right)^{2}
\end{aligned}
$$

Taking $\tau$ to be the unit measure on 1 , we similarly get $E\left[\left(\Delta T_{k}\right)^{2}\right]=\left(E\left[\Delta T_{k}\right]\right)^{2}=\left(t_{\vec{a}}\right)^{2}$.

Using this, we get

$$
\begin{aligned}
E\left[\left(T_{k}-\Upsilon_{k}\right)^{2}\right] & =\sum_{l=1}^{k} E\left[\left(\Delta T_{l}-\Delta \Upsilon_{l}\right)^{2}\right]+\sum_{l=1}^{k} \sum_{m>l} E\left[\Delta T_{l}-\Delta \Upsilon_{l}\right] \cdot E\left[\Delta T_{m}-\Delta \Upsilon_{m}\right] \\
& =\sum_{l=1}^{k} E\left[\left(\Delta T_{l}-\Delta \Upsilon_{l}\right)^{2}\right] \\
& <\sum_{l=1}^{k} E\left[\left(\Delta T_{l}\right)^{2}\right]+E\left[\left(\Delta \Upsilon_{l}\right)^{2}\right] \\
& \leq \sum_{l=1}^{k}\left(E\left[\left(\Delta T_{l}\right)\right]\right)^{2} \cdot 3 \\
& \leq\left(\sum_{l=1}^{k} E\left[\left(\Delta T_{l}\right)^{2}\right]\right) \cdot\left(t_{\max _{1}}\right)^{N} \cdot 3
\end{aligned}
$$




$$
\leq E\left[T_{k}\right] \cdot\left(t_{\max }\right)^{N} \cdot 3 \simeq 0
$$

for $\mathrm{k}$ so small that $E\left[T_{k}\right]$ is finite.

Thus $T_{k} \simeq \Upsilon_{k}$ a.s. for $\mathrm{k}$ so small that $E\left[T_{k}\right]$ is finite. So, if $k(t)=\max \left\{k \mid \Upsilon_{k} \leq t\right\}$,

$$
\begin{aligned}
Z^{(N)}(\omega, t) & \simeq Y^{(N)}(\omega, t) \\
& =B^{(N)}(\omega, k(t)) \\
& =X^{(N)}\left(\omega, T_{k(t)}\right) \\
& \simeq X^{(N)}(\omega, t)
\end{aligned}
$$

The last $\simeq$ by 2.4 .1 .

So, by transience of $\simeq$, proposition 2.4.1 and lemma 3.1.1 applies to $Z^{(N)}, N \in{ }^{*} N-N$, too.

\subsection{The Markov Property}

Lemma 3.3.1 There exists a measure $\mu_{n}$ on $\mathcal{P}\left(F^{(n)} \cup E^{(n)}\right)$ that is invariant under $Z^{(n)}$, i.e., if we let $P\left\{\omega: Z^{(n)}(0, \omega) \in A\right\}=\mu_{n}(A)$, then

$$
P\left\{\omega: Z^{(n)}(t, \omega) \in A\right\}=\mu_{n}(A)
$$

for all $t \in \mathbb{R}^{+}$.

Proof: We begin by defining this $\mu_{n}$ :

$$
\mu(x)= \begin{cases}\frac{1}{2}\left(r_{\vec{a}}^{D}-\Delta t \cdot r_{\vec{a}}^{-R}\right) & \text { if } x \in E^{(n)} \text { and } x \in C_{\vec{a}} \\ \frac{1}{2} \sum_{\substack{a \in A^{n}: \\ x \in C_{\vec{d}}}} \Delta t \cdot r_{\vec{a}}^{-R} & \text { if } x \in F^{(n)}\end{cases}
$$

To prove the lemma, all we have to show is that $P\left\{\omega: Z^{(n)}(\Delta t, \omega) \in A\right\}=\mu_{n}(A)$, and the rest follows by induction. We need only show that the measure of a point in $F^{(n)} \cup E^{(n)}$ remains the same. First, consider $x \in F^{(n)}$. From the definitions we see that

$$
\begin{aligned}
& P\left\{\omega: Z^{(n)}(\Delta t, \omega)=x\right\}=\sum_{\substack{d \in A^{n} \\
x \in C_{\vec{a}}}}\left(p_{n}\left(U_{\vec{a}}(x), \vec{a}\right) \cdot \mu_{n}\left(U_{\vec{a}}(x)\right)+\frac{\Delta t}{t_{\vec{a}}} \mu_{n}\left(\psi\left(\vec{a}, U_{\vec{a}}(x)\right)\right)\right)
\end{aligned}
$$

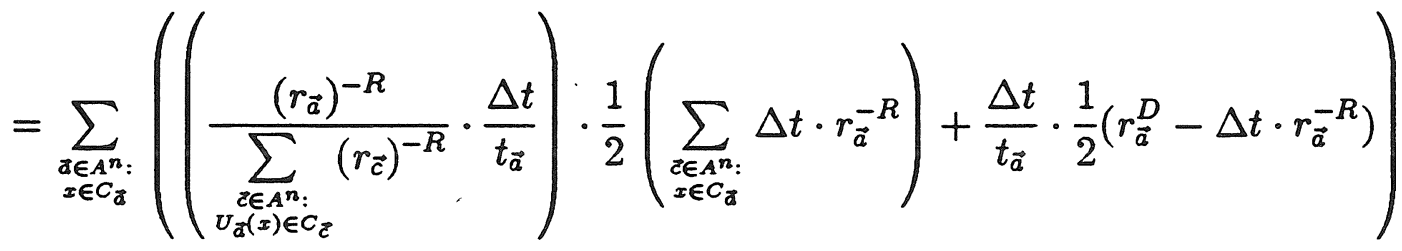




$$
\begin{aligned}
& =\frac{\Delta t}{t_{\vec{a}}} \cdot \sum_{\substack{d \in A^{n}: \\
x \in C_{\vec{a}}}}\left(\frac{1}{2} \Delta t \cdot r_{\vec{a}}^{-R}+\frac{1}{2}\left(r_{\vec{a}}^{D}-\Delta t \cdot r_{\vec{a}}^{-R}\right)\right) \\
& =\frac{1}{2} \sum_{\substack{d \in A^{n}: \\
x \in C_{\vec{d}}}} \frac{\Delta t}{\left(r_{\vec{a}}\right)^{T}} \cdot r_{\vec{a}}^{D}=\frac{1}{2} \sum_{\substack{\vec{a} \in A^{n}: \\
x \in C_{\vec{d}}}} \Delta t \cdot r_{\vec{a}}^{-R}
\end{aligned}
$$

where the last equality follows from the fact that that $T=D+R$, as according to theorem 1.4.2. Second, if $x \in E^{(n)}$, then

$$
\begin{aligned}
& P\left\{\omega: Z^{(n)}(\Delta t, \omega)=x\right\}=\left(1-\frac{\Delta t}{t_{\vec{a}}}\right) \cdot \mu_{n}(x)+p_{n}\left(\psi^{-1}(x)\right) \cdot\left(1-\frac{\Delta t}{t_{\vec{a}}}\right) \cdot \mu_{n}\left(\left(\psi^{-1}(x)\right)_{2}\right) \\
& =\frac{1}{2}\left(1-\frac{\Delta t}{t_{\vec{a}}}\right) \cdot\left(\left(r_{\vec{a}}\right)^{D}-\Delta t \cdot r_{\vec{a}}^{-R}+\frac{\left(r_{\vec{a}}\right)^{-R}}{\sum_{\substack{z \in A^{n}: \\
U_{\vec{a}}(x) \in C_{\vec{c}}}}\left(r_{\vec{c}}\right)^{-R}} \cdot \sum_{\substack{\vec{c} \in A^{n}: \\
v_{\vec{d}}(x) \in C_{\vec{c}}}} \Delta t \cdot\left(r_{\vec{c}}\right)^{-R}\right) \\
& =\frac{1}{2}\left(1-\frac{\Delta t}{t_{\vec{a}}}\right) \cdot\left(\left(r_{\vec{a}}\right)^{D}-\Delta t \cdot r_{\vec{a}}^{-R}+\Delta t \cdot\left(r_{\vec{c}}\right)^{-R}\right)=\frac{1}{2}\left(r_{\vec{a}}^{D}-\Delta t \cdot r_{\vec{a}}^{-R}\right)
\end{aligned}
$$

Recalling from section 1.2 the geometric measure on $\mathbf{K}, \mu$, and norming this so that $\mu(\mathbf{K})=1$, we have

Corollary 3.3.2 The geometric measure on $\mathbf{K}, \mu$, is invariant under the geometric diffusion on $\mathbf{K}, B$.

Proof: $\mu$ is the standard part of $\mu_{N}$ for infinite $N$, and $B={ }^{\circ}\left(Z^{(N)}\right)$.

Theorem 3.3.3 $B$ is a strong Markov process.

Proof: Combining [1, Theorem 5.4.17] with [1, definition 5.4.13], and adapting it to our setting, we get the statement:

Theorem Assume $S_{0}$ is a hyperfinite subset of ${ }^{*} Y$ for some compact space $Y$, and let $X: \Omega \times T \rightarrow S$ be an $S$-continuous Markov process on the hyperfinite time line $T$ preserving some measure on $S_{0}$, such that

$$
x \simeq y \rightarrow P_{L}^{x}\left\{\left.\omega\right|^{\circ} X(\omega, t) \in M\right\}=P_{L}^{y}\left\{\left.\omega\right|^{\circ} X(\omega, t) \in M\right\}
$$

for all finite $t$ and all Borel sets $M$. If $x$ is the standard part of $X$, then $\left\langle\Omega,\left\{\mathcal{F}_{t}\right\}_{t \in \mathbb{R}},\left\{\bar{P}_{y}\right\}_{y \in Y}, x\right\rangle$ is a strong Markov process. 
$P_{L}$ is here the Loeb measure of the internal measure $P$.

Let $t$ be fixed, and let $Z_{t}(\cdot)=Z^{(N)}(\cdot, t)$. The functions $Z_{t}: \Omega \rightarrow E^{(N)} \cup F^{(N)}$ and st: $E^{(N)} \cup F^{(N)} \rightarrow \mathbf{K}$ are measurable. So, ${ }^{\circ} Z_{t}: \Omega \rightarrow \mathbb{K}$ is measurable, too. Define

$$
\nu_{z}(\cdot)=P_{L}^{z}\left({ }^{\circ} Z_{t}^{-1}(\cdot)\right)=P_{L}^{z}\left\{\left.\omega\right|^{\circ} Z_{t}(\omega) \in(\cdot)\right\}
$$

$\nu_{z}$ is a Radon probability measure on $K$, by the measurability of ${ }^{\circ} Z_{t}$. The demand in equation 3.2 can be restated that if $x \simeq y$, then $\nu_{x}(B)=\nu_{y}(B)$ for all Borel sets $B$. Now, since $\mathbb{K}$ is a compact metric space, $\nu_{z}(B)=\sup \left\{\nu_{z}(C) \mid C \subset B, C\right.$ compact $\}$, so it suffices to show $\nu_{x}(C)=\nu_{y}(C)$ for the compact subsets $C$ of $\mathbf{K}$.

Fix $C \subset \mathbb{K}$, compact. Define the functions

$$
f_{n}(x)= \begin{cases}1 & \text { for } x \in C \\ 0 & \text { for } x: d(x, C) \geq \frac{1}{n} \\ 1-n \cdot d(x, C) & \text { otherwise }\end{cases}
$$

Now,

$$
\nu_{z}(C)=\int \chi_{C} d \nu_{z}=\lim _{n \rightarrow \infty} \int f_{n} d \nu_{z}
$$

where the last equality follows from Dominated Convergence. So, it suffices to show that

$$
E^{x}\left[f_{n}\left({ }^{\circ} Z_{t}(\omega)\right]=E^{y}\left[f_{n}\left({ }^{\circ} Z_{t}(\omega)\right)\right]\right.
$$

for $x \simeq y$. But this is a trivial consequence of lemma 3.1.1 and the continuity of $f_{n}$. 


\section{Chapter 4}

\section{Relaxing the Axioms}

In mathematics, we seek for the most general solutions, and thus prefer the weakest possible axioms that will still yield the wanted results. In our case, there are three axioms that are natural candidates for being removed/relaxed:

- Symmetry

- Simplicity

- Niceness

In the case of Simplicity, we already know that it can be relaxed when the cells are of the same size, as this in essence is the work of Lindstrøm [12]. I believe a synthesis of it and this thesis is within the scope of the techniques applied here.

Removing Symmetry/Niceness, on the other hand, is a problem I do not know has been tackled yet. What I consider a main difficulty is that we might get points on $F^{(N)}$ $\left[N \in^{*} \mathrm{~N}-\mathrm{N}\right]$ where the ratio of time to cross some two different $N$-cells connecting to that point, is infinite or infinitesimal.

\subsection{Removing Niceness}

The results from the previous chapters work equally well for fractals with Axiom 6, Niceness, removed, as long as the contraction factors from Axiom 1, the $r_{a}$, are equal and independent of $a$. I will, as representatives of this class of fractals choose a subclass that exhibits the peculiar properties of the full class in a not so difficult way.

The intuitive describtion of the spaces we will look at, is that they have a generator given by the following:

$$
\text { Let } X=\{1,2, \ldots, n\} \times[0,1]=\{(i, x): i \in\{1, \ldots, n\}, x \in[0,1]\}
$$


and then define the equivalence relation $\sim$ by

$$
(i, x) \sim(j, y) \leftrightarrow x=y \wedge\left(x=0 \vee x=\frac{1}{2} \vee x=1\right)
$$

Our generator will be $G=X / \sim$, with the sets $\left(i,\left[0, \frac{1}{2}\right]\right) / \sim$ and $\left(i,\left[\frac{1}{2}, 1\right]\right) / \sim, i \in$ $\{1, \ldots, n\}$ representing the next generation.

The exact construction of these spaces is given by the method in Part II, when we set

$$
\begin{gathered}
A=\{1,2, \cdots, n\} \\
\mu_{1}(i)=\frac{1}{|A|} \\
\sim \text { satisfies }
\end{gathered}
$$

$$
\begin{aligned}
& i_{f} \sim j_{f} \text { if } i, j \leq n \\
& i_{f} \sim j_{f} \text { if } i, j>n \\
& \tau_{i}\left(j_{f}\right)=\tau_{j}\left(i_{f}\right) \text { if } i_{f} \not j_{f}
\end{aligned}
$$

where $i_{f}$ is the fixed point of $\tau_{i}$.

It is not difficult to see that the whole of the discussion in chapter 1 carries through to the spaces we are now considering, with the exception of the proof that the Hausdorff dimension equals the geometric dimension, and that $\rho$ can be extended to the whole of $\mathbf{K}$, thus making a metric. In fact, as we will see, $\rho$ is provably not a metric when $n \neq 1$.

Now, calculating $D$ and $R$ is an easy excercise:

$$
D=\frac{\log (2 n)}{\log (2)} . \text { Similarly } R=1-\frac{\log (n)}{\log (2)}
$$

We see that for $n=2$, we have $R=0$, and for $n>2$, we get a negative $R$. That means that for $n>2$, resistance diverges to infinity when the distance between points go to zero, rather than converge.

For time scaling, we get, by using 1.4.2, T=R+D=2, independent of $n$.

The results of chapter 2 are rather straight-forward, as the $\mathcal{J}$ has only one element, and as is not too difficult to see, the limit time distribution, $\tau$, is the same, independent of $n$. So, since for $n=1$ we have Brownian Motion on the unit interval, we know this $\tau$ explicitly, and the projection, $B^{\prime}(\omega, t)=d(\underline{0}, B(\omega, t))$ is Brownian Motion on the unit interval.

The Markov property is not a problem with these spaces, since already initial the random walks are Markov, and we can apply the theorem that says standard parts are Markov directly.

Now, knowing that the results ${ }^{1}$ from the previous chapters apply in our context, too, we can state the following corollary of 2.4.1,

\footnotetext{
${ }^{1}$ Except, as stated, that $\rho$ can be extended to a metric, and that $\mathcal{H}-\operatorname{dim}(\mathbb{K})=D$.
} 
Corollary 4.1.1 The Hausdorff dimension of a Brownian path is less than or equal to $T$, a.s.

Proof: $B(\cdot, \omega)$ satisfies the Hölder condition of exponent $\frac{1}{T+\varepsilon}$ for all $\varepsilon>0$, by the above proposition, and so, by [9, Proposition 2.3], the dimension of the Brownian path is less than or equal to $T+\varepsilon$ for all $\varepsilon>0$.

It seems rather- likely, though hard to prove, that 1.2.2 holds for all Simple Fractal spaces, so we state

Conjecture 4.1.2 The self-similarity dimension of $\mathbf{K}, D$, is its Hausdorff dimension also when Niceness is removed, and $0<\mathcal{H}^{D}(\mathbf{K})<\infty$.

Under this conjecture, it is easy to prove

Proposition 4.1.3 When $n>2$ the paths of $B(\omega, \cdot)$ do not cover the whole of $\mathbb{K}, L(P)$. a.s.

Proof: $n>2$ means $D>2$. By 1.4.2 and 4.1.1, the Hausdorff dimension of a path $B(\omega, \cdot)$ is $L(P)$-a.s. less than $D$, which by 4.1 .2 is the Hausdorff dimension of $\mathbf{K}$.

I will leave off further study of these spaces until later, but will mention that if we take the relation $S=2 \frac{D}{T}$, where $S$ is the spectral dimension of $\mathbf{K}$, to be true, then we can construct Fractal spaces with arbitrarily large spectral dimensions, as in our case, $S=D$, and $D=\frac{\log 2 n}{\log 2}$.

\subsection{Relaxing Simplicity}

I expect that the relaxation of Simplicity into "Finite Ramification" in the general case will be possible by using the techniques I have applied.

We can relax the Simplicity axiom into

Axiom 3 [Finite Ramification] The set $F^{(0)}=\bigcup_{\substack { i \in A \\ \begin{subarray}{c}{j \in A \\ j \neq i{ i \in A \\ \begin{subarray} { c } { j \in A \\ j \neq i } }\end{subarray}} \phi_{i}^{-1}\left(\phi_{i}(\mathbf{K}) \cap \phi_{j}(\mathbf{K})\right)$ is finite, and the elements of $F^{(0)}$ are all fixed points for some $\phi_{i}, i \in A$.

This requires that we alter the Symmetry axiom to fit the new situation ${ }^{2}$ :

\footnotetext{
${ }^{2}$ This characterization of symmetry is due to Dr. K.R. Wicks
} 
Axiom 5 [Symmetry] For all $x, y \in F^{(0)}$ there exists a self-inverse isometry $s_{x y}$ such that

$$
\begin{aligned}
& \text { (i) } s_{x y}(x)=y \text { and } s_{x y}\left(F^{(0)}\right)=F^{(0)} \\
& \text { (ii) } \forall i \in A \exists j \in A\left(s_{x y} \circ \phi_{i} \circ s_{x y}^{-1}=\phi_{j}\right) \\
& (i i i) \forall z \in \mathbb{K}\left(d(z, x)=d(z, y) \rightarrow s_{x y}(z)=z\right)
\end{aligned}
$$

To make our construction of Brownian Motion work for these spaces, I consider the greatest obstacle to be the resistance. It surely has to be geometric, so that it has a scaling exponent $R$. But whether it is unique, and if the vector of probabilities of how likely the process is to hit one site of $F^{(0)}$ before it hits another, is unique, is a problem too big to be answered in this tentative discussion. Solving it, however, would probably involve use of the techniques of Barlow [4].

For our purposes, it is sufficient to consider the case that we have a satisfactory resistance function, which is geometric, and preserved under isometries like for instance the symmetries above, and that $T=D+R$.

The results of chapter 2 then follow straight through, as we are considering triplets $(\underline{i}, \underline{j}, \vec{a})$ as indexes on our transition times, so that we are not only considering the starting point and the cell crossed, but at which of its ramification points we end up. Chapter 3 follows by making some adjustments to the fact that $F^{(0)}$ is larger than 2 when we construct $Z$ and the invariant measure.

So, all in all, I consider the relaxation of Simplicity as a feasible next expansion in the study of Brownian motion on fractals. As seen above, the biggest task will be that of assigning unique resistances. Perhaps the solution to Brownian motion on fractals in general lies in the study of resistances. In any case, I think fractal resistance will be a subject worth looking into. 
Part II

The Space 


\section{Chapter 5}

\section{Simple fractal spaces}

In this chapter I will construct the spaces on which I have worked in the previous chapter. It will hopefully give better insight to what Simple Fractal spaces really are.

\subsection{The topological measure space $A^{\mathrm{N}}$}

Definition 4 With $|\cdot|$ denoting cardinality, let $A$ be such that $2 \leq|A|<|\mathbb{N}|$. For every $n \in \mathbb{N}$, we define $A^{n}=\left\{\left(a_{1}, \ldots, a_{n}\right): a_{i} \in A\right\}$, the set of all $n$-sequences of elements in $A$, and let $A^{\mathbb{N}}=\left\{\left(a_{1}, a_{2}, \ldots, a_{i}, \ldots\right): a_{i} \in A\right\}$, the set of all infinite sequences of elements in $A$, and let $\tau_{i}\left(a_{1}, a_{2}, \ldots\right)=\left(i, a_{1}, a_{2}, \ldots\right), \tau_{i}\left(a_{1}, a_{2}, \ldots, a_{n}\right)=\left(i, a_{1}, a_{2}, \ldots, a_{n}\right)$. If $\vec{a}=\left(a_{1}, a_{2}, \ldots, a_{n}\right) \in A^{n}$, let $\tau_{\vec{a}}=\tau_{a_{1}} \circ \tau_{a_{2}} \circ \cdots \circ \tau_{a_{n}}$.

We endow $A$ with a discrete topology, and $A^{n}$ and $A^{\mathbb{N}}$ with the product topology. The topology on $A^{n}$ will then itself be discrete. The topology on $A^{\mathbb{N}}$ is generated by $\mathcal{G}=\left\{p r_{n}^{-1}(k): k \in A, n \in \mathbb{N}\right\}$, where $p r_{n}\left(a_{1}, a_{2}, \ldots\right)=a_{n}$. If we let $\psi_{n}: A^{\mathbb{N}} \rightarrow A^{n}$ be defined by $\psi_{n}\left(a_{1}, a_{2}, \ldots\right)=\left(a_{1}, a_{2}, \ldots, a_{n}\right)$, we see that if $\vec{a}=\left(a_{1}, a_{2}, \ldots, a_{n}\right) \in A^{n}$, we have $\psi_{n}^{-1}(\vec{a})=\left\{\left(s_{1}, s_{2} \ldots\right) \in A^{\mathbb{N}}: s_{i}=a_{i}, 1 \leq i \leq n\right\}=\bigcap_{i=1}^{n} p r_{n}^{-1}\left(a_{i}\right)$ in the topology on $A^{\mathbb{N}}$. If $\vec{b} \in A^{n}$, we let $[\vec{b}]=\psi_{n}^{-1}(\vec{b})$ be called an $(n-)$ cylinder in $A^{\mathbb{N}}$. Let $\mathcal{O}$ be an open neighbourhood of $x=\left(x_{1}, x_{2}, \ldots\right) \in A^{\mathbb{N}}$. Then $\mathcal{O}$ has to be a union of finite intersections of generating sets, so $x$ will have to be in one of these finite intersections of generating sets. Let $\bigcap_{i=1}^{k} p r_{n_{k}}^{-1}\left(x_{n_{k}}\right)$ be it, and let $n=\max \left\{n_{k}\right\}$. Then the intersection will contain $\bigcap_{i=1}^{n} p r_{i}^{-1}\left(x_{i}\right)=\psi_{n}^{-1}\left(x_{1}, x_{2}, \ldots, x_{n}\right)$, and we have shown that the cylinders make a basis for the topology on $A^{\mathbb{N}}$.

Since $A$ is a finite set, $A$ is compact. And since products of compact spaces are compact, both $A^{n}$ and $A^{\mathbb{N}}$ are compact. If $x=\left(x_{1}, x_{2}, \ldots\right), y=\left(y_{1}, y_{2}, \ldots\right) \in A^{\mathbb{N}}$, let $k$ be such that $x_{k} \neq y_{k}$. Then $\left(x_{1}, \ldots, x_{k}\right) \neq\left(y_{1}, \ldots, y_{k}\right)$, whereupon $\left[\left(x_{1}, \ldots, x_{k}\right)\right]$ and $\left[\left(y_{1}, \ldots, y_{k}\right)\right]$ are disjoint neighbourhoods of $x$ and $y$, respectively: $A^{\mathbb{N}}$ is Hausdorff. 
Let $\mathcal{B}_{1}$ be the Borel- $\sigma$-algebra on $A$, and give $\left(A, \mathcal{B}_{1}\right)$ a measure $\mu_{1}$, such that $\mu_{1}(A)=1$, and if $B \in \mathcal{B}_{1}, \mu_{1}(B)=0 \Leftrightarrow B=\emptyset$. From this measure we will make product measures on $A^{n}$ and $A^{\mathbb{N}}$. Let $\mathcal{B}_{n}$ be the Borel- $\sigma$-algebra on $A^{n}$. Then $\mathcal{B}_{n}=\mathcal{P}\left(A^{n}\right)$, since $A^{n}$ has discrete topology. Since $\psi_{n}^{-1}\left(A^{n}\right)=A^{\mathbb{N}}$, and $\psi_{n}^{-1}(\emptyset)=\emptyset, \psi_{n}^{-1}\left(\mathcal{B}_{n}\right)$ is a $\sigma$-algebra on $A^{\mathbb{N}}$. Since $\psi_{n-1}^{-1}\left(\mathcal{B}_{n-1}\right) \subset \psi_{n}^{-1}\left(\mathcal{B}_{n}\right), \psi_{n}^{-1}\left(\mathcal{B}_{n}\right)$ is an increasing sequence of $\sigma$-algebras on $A^{\mathbb{N}}$. Let $\mathcal{B}_{\mathbb{N}}$ be the $\sigma$-algebra generated by $\bigcup_{i=1}^{\infty} \psi_{n}^{-1}\left(\mathcal{B}_{n}\right)$. But $\bigcup_{i=1}^{\infty} \psi_{n}^{-1}\left(\mathcal{B}_{n}\right)=\left\{[\vec{b}]: \exists n \in N\right.$ s.a. $\left.\vec{b} \in A^{n}\right\}$, the neighbourhood basis for the topology on $A^{\mathbb{N}}$, so $\mathcal{B}_{\mathbb{N}}$ is generated by the topology on $A^{\mathbb{N}}$, and is thus the Borel- $\sigma$-algebra on $A^{\mathbb{N}}$. Let $\mu_{n}$ be the product measure on $\left(A^{n}, \mathcal{B}_{n}\right)$ of $\mu_{1}$ with itself $n$ times. Since $\mu_{1}$ is a probability measure, there exists, according to Parthasarathy [13] a natural product probability measure, $\mu_{\mathbb{N}}$, on $\left(A^{\mathbb{N}}, \mathcal{B}_{\mathbb{N}}\right)$ such that

Proposition 5.1.1 $\mu_{N} \psi_{n}^{-1}=\mu_{n}, \forall n \in \mathbb{N}$

Lemma 5.1.2 If $S \in \mathcal{B}_{\mathbb{N}}$, then $\mu_{\mathbb{N}}(S)=\sum_{i \in A} \mu_{1}(i) \cdot \mu_{N}\left(\tau_{i}^{-1}(S)\right)$

Proof: Let $\pi(\cdot)=\sum_{i \in A} \mu_{1}(i) \cdot \mu_{\mathbb{N}}\left(\tau_{i}^{-1}(\cdot)\right)$. Then $\pi$ is a measure on $\left(A^{\mathbb{N}}, \mathcal{B}_{N}\right)$. We will apply Caratheodory's extention theorem to show the equality of $\pi$ and $\mu_{N}$. It it will be sufficient to show equality on $\bigcup_{i=1}^{\infty} \psi_{n}^{-1}\left(\mathcal{B}_{n}\right)$. But that equality, again, follows from equality on the cylinders, and the lemma follows.

Corollary 5.1.3 $\mu_{\mathbb{N}} \circ \tau_{\vec{a}}(\cdot)=\mu_{\mathbb{N}}([\vec{a}]) \cdot \mu_{N}(\cdot)$

\subsection{The quotient space $K$}

Given $A$ and its product spaces $A^{n}$ and $A^{N}$ from the last section, we define a new space, $\mathbf{K}=A^{\mathbb{N}} / \sim$, where $\sim$ is an equivalence relation. We endow $\mathbf{K}$ with quotient topology and -measure. By imposing certain demands on $\sim$ and $\mu_{\mathbb{N}}\left(\mu_{1}\right)$, we will make our self-similar spaces. The construction is inspired by Bandt [2] and Lindstrøm [12]. Bandt defines $\mathbf{K}$ as a " $\tau$-invariant factor if $x \sim y \Leftrightarrow \tau_{i}(x) \sim \tau_{i}(y), \forall i \in A$, or, as Bandt demonstrates to be equivalent, we have homeomorphisms $f_{i}: \mathbf{K} \rightarrow \mathbf{K}$ such that $f_{i} \circ p=p \circ \tau_{i}$, where $p$ is the canoncal mapping from $A^{\mathbb{N}}$ to $\mathbf{K}$. He constructs the graph $G$ with corner set $A$, and connects two corners, $i$ and $j$ with an edge if there are points $\left(i, x_{1}, x_{2}, \ldots\right) \sim\left(j, y_{1}, y_{2}, \ldots\right)$, and later shows that either both or none of $\mathbf{K}$ and $G$ are connected.

Lindstr $\varnothing \mathrm{m}[12]$ defines the set $F_{0}$ of fixed points of contractions $\phi_{i}$. Let $x_{i}$ be the fixed point of $f_{i}$, and $F=\left\{x_{i}\right\}$. We then define with him $F^{(0)}$, "the set of essential fixed points", as the set of $x \in F$ such that $\exists y \in F$ such that $\exists i, j \in A, i \neq j$, such that $f_{i}(x)=f_{j}(y)$. By using these definitions, we will define the spaces we will work on. They will be the $\mathbf{K}$ where 
Axiom $1 \tau$-invariance: $\mathbf{K}$ is a $\tau$-invariant factor such that the inverse images of points in $\mathbf{K}$ are closed sets in $A^{\mathbb{N}}$.

Axiom 2 Finite Ramification: If $i, j \in A, i \neq j$, then $f_{i}(\mathbf{K}) \cap f_{j}(\mathbf{K})=f_{i}\left(F^{(0)}\right) \cap f_{j}\left(F^{(0)}\right)$.

Axiom 3 Connectedness: The graph $G$, as defined above, is connected.

Axiom 4 Simplicity: $\left|F^{(0)}\right|=2$

The two elements of $F^{(0)}$ we will call $\underline{0}$ and $\underline{1}$.

Axiom 5 Symmetry: There is a mapping $U_{1}: A \rightarrow A$ such that

1. $U_{1}^{2}=i d$

2. $\left|f_{i}(\mathbf{K}) \cap f_{j}(\mathbf{K})\right|=\left|f_{U_{1}(i)}(\mathbf{K}) \cap f_{U_{1}(j)}(\mathbf{K})\right| \forall i, j \in A$

3. $\underline{0} \in C_{i} \Rightarrow \underline{1} \in C_{U_{1}(i)}$

4. $\mu_{1} \circ U_{1}=\mu_{1}$

Axiom 6 Niceness: $\left|\left\{a \in A: \underline{0} \in C_{a}\right\}\right|=\left|\left\{a \in A: \underline{1} \in C_{a}\right\}\right|=1$.

The spaces that satisfy the axioms $1-5$ and have metric given by theorem 5.5 .2 will be called Simple Fractal Spaces.

When we also include Axiom 6, we get the spaces we have been working on in chapters 1 through 3. However, as this axiom is the prime candidate for removal, I did not want to include it in the definition of what a Simple Fractal space is.

\subsection{The topological space $(\mathbf{K}, \Theta)$}

By Axiom 3 and by Bandt [2] we immediately get

Proposition 5.3.1 The space $\mathbf{K}$ with the quotient topology is connected.

Definition 5 Let $\Theta$ be the name of the quotient topology on $\mathbf{K}$. We define an $n$-cell in $\mathbf{K}$ to be the image of an $n$-cylinder in $A^{\mathbb{N}}$ by $p$.

We see that the $n$-cells correspond one-to-one with the $n$-cylinders, that correspond one-to-one with the elements in $A^{n}$. Thus we write $C_{\vec{b}}$ for $p([\vec{b}])$. It is easy to see that if we let $\vec{x}=\left(x_{1}, x_{2}, \ldots, x_{n}\right)$, and $f_{\vec{x}}=f_{x_{1}} \circ f_{x_{2}} \circ \cdots \circ f_{x_{n}}$, we have $C_{\vec{x}}=f_{\vec{x}}(\mathbf{K})$. Since the $f_{\vec{x}}$ are homeomorphisms for all $\vec{x}$, we have, for each $\vec{x}$, a 1-1 mapping between $C_{\vec{x}}$ and $\mathbf{K}$.

Proposition 5.3.2 $(\mathrm{K}, \Theta)$ is compact. 
Proof: $A^{\mathbb{N}}$ is compact, and $p: A^{\mathbb{N}} \rightarrow \mathrm{K}$ is continuous.

Definition 6 If $n \in \mathbb{N}$ we define $F^{(n)}=\bigcup_{i \in A} f_{i}\left(F^{(n-1)}\right)$. Then $F^{(n)}=\bigcup_{\vec{a} \in A^{n}} f_{\vec{a}}\left(F^{(0)}\right)$. Let $F^{(\infty)}=\bigcup_{n \in \mathbb{N}} F^{(n)}$

Definition 7 Let $U^{n}(x)$ be the union of all $n$-cells where $x$ is an element: $U^{n}(x)=$ $\bigcup_{a \in p^{-1}(x)} C_{\psi_{n}(a)}$

Lemma 5.3.3 $\mathcal{O}$ is a neighbourhood of $x$ iff $\mathcal{O} \supset U^{n}(x)$ for some natural number $n$.

Proof: That $U^{n}(x)$ is a neighbourhood of $x$ is trivial by the definition of $\Theta$ and $U^{n}(x)$. For $x \in \mathbf{K}$, let $\mathcal{O}$ be a neighbourhood of $x$. Then, by the continuity of $p, p^{-1}(\mathcal{O})$ is a neighbourhood of each of the elements in $p^{-1}(x)$. But then, given one such element, $y$, there exists a cylinder $S, S \subset p^{-1}(\mathcal{O})$, which is a neighbourhood of $y$. Let $P$ be the set of such cylinders. Then $P$ is an open covering of $p^{-1}(x)$, and by Axiom $1, p^{-1}(x)$ is closed, and thereby compact. We can thus reduce $P$ to a finite covering, $P^{\prime} . \cup_{S \in P^{\prime}} S$ will then be a neighbourhood of each of the elements in $p^{-1}(x)$. Each cylinder $S$ is an $n$-cylinder for some $n \in N$. Let $k$ be the largest such $n$. Since, when $m>n$, an $n$-cylinder is the union of a finite collection of $m$-cylinders, $\bigcup_{S \in P^{\prime}} S$ is a finite union of $k$-cylinders, and $p\left(\bigcup_{S \in P^{\prime}} S\right) \supset p\left(\bigcup_{a \in p^{-1}(x)}\left[\psi_{k}(a)\right]\right)=U^{k}(x)$. Since $p^{-1}(\mathcal{O}) \supset \bigcup_{S \in P^{\prime}} S$, we must have $\mathcal{O} \supset U^{k}(x) . \square$

Corollary 5.3.4 $F^{(\infty)}$ is dense in $\mathbf{K}$.

Lemma 5.3.5 $\bigcap_{n \in \mathbb{N}} C_{\psi_{n}(x)}=p(x)$.

Proof: $p(x) \in \bigcap_{n \in \mathbb{N}} C_{\psi_{n}(x)}$. Suppose $\bigcap_{n \in \mathbb{N}} C_{\psi_{n}(x)}$ consists of more than one point. Then, for each point $z$ in the set, and for each $n \in \mathbb{N}$, there exists an element $z_{n} \in\left[\psi_{n}(x)\right]$ such that $p\left(z_{n}\right)=z$. Then $\left\{z_{n}\right\}_{n \in \mathbb{N}}$ is a subset of the equivalence class that is mapped onto $z$. But $\lim _{n \rightarrow \infty}\left(z_{n}\right)=x$, so that $z_{n} \sim x$, by Axiom 1. Thus $z=x$.

Lemma 5.3.6 If $x \in F^{(0)} \cap C_{\vec{d}}$, then $x \in f_{\vec{d}}\left(F^{(0)}\right)$.

Proof: Let us first see that if $x \in F^{(0)} \cap C_{a}, a \in A$, then $x \in f_{a}\left(F^{(0)}\right)$. If $x$ is an element of only one 1-cell, $C_{a}=f_{a}(\mathbf{K}), x$ has to be the fixed point of $f_{a}$, by the definition of $F^{(0)}$. But then $x=f_{a}(x) \in f_{a}\left(F^{(0)}\right)$. If not, there exists an other 1-cell, $C_{b}$, such that $x \in C_{b} \cap C_{a}$. But by Axiom 2, $x \in f_{b}\left(F^{(0)}\right) \cap f_{a}\left(F^{(0)}\right) \subset f_{a}\left(F^{(0)}\right)$. 


\section{CHAPTER 5. SIMPLE FRACTAL SPACES}

We will proceed inductively. Let $x \in F^{(0)} \cap C_{\vec{d}}, \vec{d} \in A^{n}$, and assume the lemma for $\vec{c} \in A^{m}, m<n$. Then $x \in C_{\left(d_{1}, \ldots, d_{n-1}\right)} \cap F^{(0)}$, so that $x \in f_{\left(d_{1}, \ldots, d_{n-1}\right)}\left(F^{(0)}\right)$. And since $f_{\left(d_{1}, \ldots, d_{n-1}\right)}$ is a homeomorphism, $x \in f_{\left(d_{1}, \ldots, d_{n-1}\right)}\left(F^{(0)}\right) \cap f_{d}(\mathbf{K})=f_{\left(d_{1}, \ldots, d_{n-1}\right)}\left(F^{(0)} \cap C_{d_{n}}\right)=$ $f_{\left(d_{1}, \ldots, d_{n-1}\right)}\left(f_{d_{n}}\left(F^{(0)}\right)\right)=f_{\tilde{d}}\left(F^{(0)}\right)$.

Corollary 5.3.7 $F^{(n)} \supset F^{(0)}, \forall n \in \mathbb{N}$

Since homeomorphisms are 1-1, they conserve intersection. We will make use of this in an extention of Axiom 2:

Proposition 5.3.8 If $\vec{b} \neq \vec{c} \in A^{n}$, then $C_{\vec{b}} \cap C_{\vec{c}}=f_{\vec{b}}\left(F^{(0)}\right) \cap f_{\vec{c}}\left(F^{(0)}\right)$.

Proof: Assume $b_{1} \neq c_{1}$. Then $C_{\vec{b}} \cap C_{\vec{c}} \subset C_{b_{1}} \cap C_{c_{1}}=f_{b_{1}}\left(F^{(0)}\right) \cap f_{c_{1}}\left(F^{(0)}\right)$, by Axiom 2. By lemma 5.3.6 $C_{\vec{b}} \cap C_{\vec{c}} \subset f_{\vec{b}}\left(F^{(0)}\right) \cap f_{\vec{c}}\left(F^{(0)}\right)$. Let $m$ be the smallest integer such that $b_{m} \neq c_{m}$. Then we know that $C_{\left(b_{m}, \ldots, b_{n}\right)} \cap C_{\left(c_{m}, \ldots, c_{n}\right)} \subset f_{\left(b_{m}, \ldots, b_{n}\right)}\left(F^{(0)}\right) \cap f_{\left(c_{m}, \ldots, c_{n}\right)}\left(F^{(0)}\right)$. So

$$
\begin{aligned}
C_{\vec{b}} \cap C_{\vec{c}} & =f_{\left(b_{1}, \ldots, b_{m-1}\right)} \circ f_{\left(b_{m}, \ldots, b_{n}\right)}(\mathbf{K}) \cap f_{\left(b_{1}, \ldots, b_{m-1}\right)} \circ f_{\left(c_{m}, \ldots, c_{n}\right)}(\mathbf{K}) \\
& =f_{\left(b_{1}, \ldots, b_{m-1}\right)}\left[f_{\left(b_{m}, \ldots, b_{n}\right)}(\mathbf{K}) \cap f_{\left(c_{m}, \ldots, c_{n}\right)}(\mathbf{K})\right] \\
& \subset f_{\left(b_{1}, \ldots, b_{m-1}\right)}\left[f_{\left(b_{m}, \ldots, b_{n}\right)}\left(F^{(0)}\right) \cap f_{\left(c_{m}, \ldots, c_{n}\right)}\left(F^{(0)}\right)\right] \\
& =f_{\left(b_{1}, \ldots, b_{m-1}\right)} \circ f_{\left(b_{m}, \ldots, b_{n}\right)}\left(F^{(0)}\right) \cap f_{b_{1}, \ldots, b_{m-1}} \circ f_{\left(c_{m}, \ldots, c_{n}\right)}\left(F^{(0)}\right) \\
& =f_{\vec{b}}\left(F^{(0)}\right) \cap f_{\vec{c}}\left(F^{(0)}\right)
\end{aligned}
$$

Corollary 5.3.9 If $C_{\vec{d}}$ is an $m$-cell, $n \leq m$, and $x \in F^{(n)} \cap C_{\vec{d}}$, then $x \in f_{\vec{d}}\left(F^{(0)}\right)$

Proof: Assume $x \notin f_{\left(d_{1}, \ldots, d_{n}\right)}\left(F^{(0)}\right)$. Then there is an $\vec{e} \neq \vec{d} \in A^{n}$, such that $x \in$ $f_{\vec{e}}\left(F^{(0)}\right)$. But then $x \in C_{\left(d_{1}, \ldots, d_{n}\right)} \cap C_{\vec{e}}$, and by $5.3 .8 x \in f_{\left(d_{1}, \ldots, d_{n}\right)}\left(F^{(0)}\right) \cap f_{\vec{e}}\left(F^{(0)}\right) \subset$ $f_{\left(d_{1}, \ldots, d_{n}\right)}\left(F^{(0)}\right)$. Therefore $x \in f_{\left(d_{1}, \ldots, d_{n}\right)}\left(F^{(0)}\right)$.

This means that $x \in f_{\left(d_{1}, \ldots, d_{n}\right)}\left(F^{(0)} \cap f_{\left(d_{n+1}, \ldots, d_{m}\right)}(\mathbf{K})\right)=f_{\left(d_{1}, \ldots, d_{n}\right)}\left(f_{\left(d_{n+1}, \ldots, d_{m}\right)}\left(F^{(0)}\right)\right)=$ $f_{d}^{-}\left(F^{(0)}\right)$.

Corollary 5.3.10 If $n<m$, then $F^{(n)} \subset F^{(m)}$.

Lemma 5.3.11 $\left|p^{-1}(x)\right|>1 \Rightarrow x \in F^{(\infty)}$ 


\section{CHAPTER 5. SIMPLE FRACTAL SPACES}

Proof: If $a, b$ are two different elements in $p^{-1}(x)$, there is a first natural number, $n$, such that $a_{n} \neq b_{n}$, whereupon $x \in C_{\psi_{n}(a)} \cap C_{\psi_{n}(b)} \subset F^{(n)} \subset F^{(\infty)}$.

Lemma 5.3.12 $F^{(0)}$ cannot be contained in a single 1-cell.

Proof: Assume the opposite. Then we would have $F^{(0)} \subset C_{i}$ for an $i \in A$. But then $F^{(0)} \subset f_{i}\left(F^{(0)}\right)$, and inductively $F^{(0)} \subset\left(f_{i}\right)^{n}\left(F^{(0)}\right)$, so that $F^{(0)} \subset \bigcap_{n \in \mathbb{N}}\left(f_{i}\right)^{n}(\mathbf{K})$, which by lemma 5.3 .5 is a one point set, contradicting Axiom 4 .

Corollary 5.3.13 A single $(n+1)$-cell cannot contain two elements of $F^{(n)}$.

Proof: Assume the opposite. Let $\vec{a}=\left(a_{1}, \ldots, a_{n}, a_{n+1}\right)$ be such that $C_{\vec{a}}$ contains two elements, $x$ and $y$ in $F^{(n)}$. Then, by 5.3 .9 we get that $\{x, y\}=f_{\vec{a}}\left(F^{(0)}\right)$, but also $\{x, y\}=f_{\left(a_{1}, \ldots, a_{n}\right)}\left(F^{(0)}\right)$. The homeomorphic property of the $f^{\prime}$ 's then give us $F^{(0)}=f_{a_{n+1}}\left(F^{(0)}\right) \subset C_{a_{n+1}}$ contrary to lemma 5.3.12.

\section{Proposition 5.3.14 $(\mathrm{K}, \Theta)$ is Hausdorff.}

Proof: We need only show that given two different points, we can assign disjoint basis neighbourhoods to them. Let $x, y \in \mathbf{K}, x \neq y$, and let, for each $n \in \mathbb{N}, X_{n}=U^{n}(x)$, and $Y_{n}=U^{n}(y)$. We need only show that there exists an $n \in \mathbb{N}$ such that $X_{n} \cap Y_{n} \neq \emptyset$. The proof goes in three steps, each dependent on the former:

Let $n^{6}$ be a natural number such that $X_{n^{6}} \cap Y_{n^{6}}$ does not contain any $n^{6}$-cells. If there is no $n^{6}$ satisfying this, we are able to find a sequence $\{C(n)\}_{n \in \mathbb{N}}$, such that $C(n)$ are n-cells, and $C(n) \subset X_{n} \cap Y_{n}, \forall n \in N$. But according to the definition of $X_{n}$ and $Y_{n}$, we must have $x, y \in C(n), \forall n \in \mathbb{N}$, so that $x, y \in \bigcap_{n \in \mathbb{N}} C(n)$. So if $C(n) \subset C(m)$ for $n<m, \bigcap_{n \in \mathbb{N}} C(n)$ is a one-point set, according to 5.3.5, whereupon $x=y$, contrary to our assumption. If there is an $n \in \mathbb{N}$ such that $C(n+1) \not \subset C(n)$, we get that $|C(n+1) \cap C(n)|=1$, and, again contrary to our assumption, $|\{x, y\}|=\left|\bigcap_{n \in \mathbb{N}} C(n)\right| \leq 1$.

Then, since $X_{n^{\natural}} \cap Y_{n^{\natural}}$ does not contain any $n^{6}$-cells, $X_{n^{\natural}} \cap Y_{n^{\natural}}$ has to be contained in $F^{\left(n^{\natural}\right)}$, by lemma 5.3.8. This means $\left|X_{n^{4}+2} \cap Y_{n^{4}+2}\right| \leq 1$. To prove this, assume the contrary: If $X_{n^{i}+2} \cap F^{\left(n^{c}\right)} \geq 2, X_{n^{c}+1}$ will have to consist of several $\left(n^{6}+1\right)$-cells, for if not, the one $\left(n^{6}+1\right)$-cell $X_{n^{6}+1}$ consisted of would have to contain two elements of $F^{\left(n^{6}\right)}$, contrary to 5.3.13. But then $x \in F^{\left(n^{6}+1\right)}-F^{(n)}$, and some $\left(n^{6}+2\right)$-cells in $X_{n^{6}+2}$ would have to contain both $x$ and an element in $F^{\left(n^{c}\right)} \subset F^{\left(n^{c}+1\right)}$, that is 2 elements in $F^{\left(n^{4}+1\right)}$, again contrary to 5.3.13.

We can now assume that $\left|X_{n^{\natural}} \cap Y_{n^{\prime}}\right| \leq 1$. If there is no $n^{\text {" }}>n^{6}+2$ such that $X_{n}{ }^{4} \cap Y_{n^{4}}=\emptyset$, the intersection is a one-point set for all natural numbers $n$. Let $z$ be this point. Then $x$ 
and $y$ have to be different from $z$, by the construction of our system of neighbourhoods. Consider the case that $x \in F^{(\infty)}$. Then there would be an $m \in N$ such that $x \in F^{(m)}$, making an $(m+1)$-cell contain both $x$ and $z$, contrary to lemma 5.3.13. Now, we must look at when $x \notin F^{(\infty)}$. Then $x=p(a)$ for only one $a \in A^{\mathbb{N}}$, by lemma 5.3.11, such that $U^{n}(x)=C_{\psi_{n}(a)}, \forall n \in N$. But then $z \in \cap_{n \in N} C_{\psi_{n}(a)}=p(a)$ by 5.3 .5 , contrary to our assumption. Similarly for $y$, and we get $X_{n^{\mu}} \cap Y_{n^{\mu}}=\emptyset$.

\subsection{The measure space $(\mathbf{K}, \mathcal{B}, \mu)$}

Definition 8 Let $\mathcal{B}$ be the Borel- $\sigma$-algebra on $\mathbf{K}$. Then $p$ is measurable, since it is continuous. We define the measure $\mu$ on $(\mathbf{K}, \mathcal{B})$ to be $\mu=\mu_{\mathbb{N}} \circ p^{-1}$.

Lemma 5.4.1 $\mu\left(F^{(0)}\right)=0$

Proof: Let $G_{0}=p^{-1}\left(F^{(0)}\right)$. Then $G_{0} \subset p^{-1}\left(\bigcup_{i \in A} f_{i}\left(F^{(0)}\right)\right)=\bigcup_{i \in A} \tau_{i}\left(p^{-1}\left(F^{(0)}\right)\right)=\bigcup_{i \in A} \tau_{i}\left(G_{0}\right)$, and $\mu_{N}\left(G_{0}\right)=\sum_{i \in A} \mu_{N}\left(\tau_{i}\left(G_{0}\right)\right)$. Let $X=p^{-1}(\underline{0}), Y=p^{-1}(\underline{1})$. From 5.3 .12 we get that at most one of $\left(\tau_{i}(X) \subset G_{0}\right),\left(\tau_{i}(Y) \subset G_{0}\right)$ are valid, so that $\mu_{N}\left(G_{0}\right) \leq \frac{1}{2} \mu_{N}\left(\cup_{i \in A} \tau_{i}\left(G_{0}\right)\right)=$ $\frac{1}{2} \sum_{i \in A} \mu_{N}\left(\tau_{i}\left(G_{0}\right)\right)=\frac{1}{2} \mu_{N}\left(G_{0}\right)$ (lemma 5.1.2). But since $\mu_{N}\left(A^{N}\right)=1$, we must have $\mu_{\mathbb{N}}\left(G_{0}\right)=0 . \mu\left(F^{(0)}\right)=\mu_{N}\left(G_{0}\right)$.

Corollary 5.4.2 $\mu\left(F^{(\infty)}\right)=0$

Proof: $\mu\left(F^{(\infty)}\right) \leq \sum_{n \in \mathbb{N}} \mu\left(F^{(n)}\right)=\sum_{n \in \mathbb{N}} \mu_{\mathbb{N}}\left(p^{-1}\left(F^{(n)}\right)\right)=\sum_{n \in \mathbb{N}} \sum_{\vec{a} \in A^{n}} \mu_{n}(\vec{a}) \cdot \mu_{\mathbb{N}}\left(G_{0}\right)=\sum_{n \in \mathbb{N}} 0=$ 0 , by corollary 5.1 .3 .

Corollary 5.4.3 $\mu(p(S))=\mu_{\mathbb{N}}(S)$, whenever $p(S) \in \mathcal{B}$

Proof: Since $p$ is $1-1$ outside $F^{(\infty)}$ (lemma 5.3.11), we have $\mu_{\mathbb{N}}(S) \leq \mu(p(S)$ ) $=$ $\mu\left(p(S)-F^{(\infty)}\right) \leq \mu_{\mathbb{N}}(S)$

Corollary 5.4.4 $\mu\left(f_{\vec{a}}(S)\right)=\mu(S) \cdot \prod_{j=1}^{n} \mu_{1}\left(a_{j}\right), a \in A^{\mathbb{N}}, S \in \mathcal{B}, \vec{a}=\left(a_{1}, \ldots, a_{n}\right)$ 


$$
\begin{aligned}
& \text { Proof: } \mu\left(f_{\vec{a}}(S)\right)=\mu_{\mathbb{N}}\left(p^{-1} \circ f_{\vec{a}}(S)\right)=\mu_{\mathbb{N}}\left(\tau_{\vec{a}} \circ p^{-1}(S)\right)=\mu_{\mathbb{N}}\left(p^{-1}(S)\right) \cdot \prod_{j=1}^{n} \mu_{1}\left(a_{j}\right)= \\
& =\mu\left(p \circ p^{-1}(S)\right) \cdot \prod_{j=1}^{n} \mu_{1}\left(a_{j}\right)=\mu(S) \cdot \prod_{j=1}^{n} \mu_{1}\left(a_{j}\right)
\end{aligned}
$$

We have established the properties of the measure $\mu$ that we need. The following definitions and lemmas are the preliminary work for a metric based upon the measure and topology we have put on $\mathbf{K}$. The idea behind the metric is, as mentioned, Bandt's Hausdorff-measures of paths. What I will do, is I find one exponent $\Delta$, characteristic to K. Then I consider the " $n$-path"s between the two points I am to measure the distance between. An " $n$-path", or as I will call it, an $n$-chain, is what is defined in Definition 8. Each cell, $C$, in the chain is measured to have length $(\mu(C))^{\Delta}$. The length of the $n$-chain is the sum of its cells lengths. The " $n$-distance" between $x$ and $y$ is to be the infimum over such lengths for $n$-chains. Last, we define the distance between $x$ and $y$ to be the limit of the " $n$-distance" as $n$ tends to infinity.

Definition 9 We say $\vec{C}=\left(C^{1}, C^{2}, \ldots, C^{m}\right)$ is an n-chain from $x$ to $y$ when the $C^{i}$ are $n$-cells, $C^{i} \neq C^{i+1}, C^{i} \cap C^{i+1} \neq \emptyset, x \in C^{1}$ and $y \in C^{m}$. We write $C^{i} \in \vec{C}$. If $\vec{B}$ also is an $n$-chain, we write $\vec{C} \triangleleft \vec{B}$ in the case that $C \in \vec{C} \Rightarrow C \in \vec{B}$.

Lemma 5.4.5 For each chain $\vec{C} \neq \emptyset$ there is a unique $\delta \in \mathbf{R}$ such that $\sum_{C \in \vec{C}}(\mu(C))^{\delta}=1$

Proof: $0<\mu\left(C^{i}\right)<1$. If $\delta<0$, then $\sum_{C \in \vec{C}}(\mu(C))^{\delta}>1$. But we can also get $\sum_{C \in \vec{C}}(\mu(C))^{\delta}$ arbitrarily close to 0 by choosing $\delta$ large enough. $\frac{\partial}{\partial \delta} \sum_{C \in \vec{C}}(\mu(C))^{\delta}=$ $\sum_{C \in \bar{C}}\left(\ln (\mu(C)) \cdot(\mu(C))^{\delta}\right)<0$, so that $\sum_{C \in C}(\mu(C))^{\delta}$ is a continuous, monotone function of $\delta$ taking values both above and below 1. By the intermediate value theorem, there is a $\delta \in \mathbb{R}$ such that $\sum_{C \in \vec{C}}(\mu(C))^{\delta}=1$. This $\delta$ is unique by monotocity.

The $\delta$ belonging to the particular chain $\vec{C}$ will be written $\delta(\vec{C})$.

Definition $10 \mathcal{C}_{n}=\left\{\vec{C}=\left(C^{1}, C^{2}, \ldots, C^{m}\right) \mid \vec{C}\right.$ is an $n$-chain such that $C^{1}$ and $C^{m}$ contain one element each from $\left.F^{(0)}\right\}$

Definition 11 Let $\Delta(n)=\inf \left\{\delta(\vec{C}): \vec{C} \in \mathcal{C}_{n}\right\}$

Lemma 5.4.6 For all $n \in \mathbb{N}$ there is a $\vec{C} \in \mathcal{C}_{n}$ such that $\delta(\vec{C})=\Delta(n)$.

Proof: Let $\vec{C}^{\prime}=\left(C^{1}, \ldots, C^{m}\right) \in \mathcal{C}_{n}$ be such that $C^{i} \neq C^{j}, i \neq j$, and let $\vec{C}^{\prime \prime}$ be such that $\vec{C}^{\prime} \triangleleft \vec{C}^{\prime \prime}$. Then $\delta\left(\vec{C}^{\prime}\right)<\delta\left(\vec{C}^{\prime \prime}\right)$, so that $\Delta(n)=\inf \left\{\delta(\vec{C}): \vec{C}=\left(C^{1}, \ldots, C^{k}\right) \in \mathcal{C}_{n}\right.$ 
where $\left.C^{i} \neq C^{j}, i \neq j\right\}$, which is an infimum over a finite set, in other words a minimum. We can then pick an element $\delta(\vec{C})$ such that $\delta(\vec{C})=\Delta(n)$.

In the following, I will need two tools that I will call induction and reduction of chains. They will be defined as follows:

Induction: Given the $(n+1)$-chain $\vec{B}=\left(C_{\vec{x}_{1}}, \ldots, C_{\vec{x}_{m}}\right)$, where $\vec{x}_{p}=\left(x_{p}^{1} \ldots, x_{p}^{n+1}\right)$, let $\vec{I}_{1}=\left(C_{\left(x_{1}^{1}, \ldots, x_{1}^{n}\right)}\right)$. Then define inductively

$$
\vec{I}_{k+1}= \begin{cases}\vec{I}_{k} & \text { if } C_{\vec{x}_{k+1}} \subset C_{\left(x_{k}^{1}, \ldots, x_{k}^{n}\right)} \\ \vec{I}_{k} \oplus\left(C_{\left(x_{k+1}^{1}, \ldots, x_{k+1}^{n}\right)}\right) & \text { otherwise }\end{cases}
$$

We will then say that $\vec{B}$ induces the $n$-chain $\overrightarrow{I_{m}}$, and write $\overrightarrow{I_{m}}=$ ind $(\vec{B})$. If $\vec{C}=$ $(\text { ind })^{p}(\vec{B})$, we say that $\vec{B}$-induces $\vec{C}$.

Reduction: First, we define the standard chain $\vec{C}_{S}$ to be a chain in $\mathcal{C}_{1}$ such that

$$
\sum_{C \in \overrightarrow{C_{S}}}(\mu(C))^{\Delta(1)}=1
$$

Let $\overrightarrow{C_{S}}=\left(C_{a_{1}}, \ldots, C_{a_{r}}\right)$, and let the $n$-chain $\vec{B}=\left(C_{\vec{x}_{1}}, \ldots, C_{\vec{x}_{m}}\right)$, where $\vec{x}_{p}=\left(x_{p}^{1} \ldots, x_{p}^{n}\right)$. If $|\vec{B}|=1$, let $\overrightarrow{I_{1}}=\left(f_{\vec{x}_{1}}\left(\overrightarrow{C_{S}}\right)\right)$. If $|\vec{B}| \geq 2$, let

$$
\vec{I}_{1}=\left\{\begin{array}{l}
\left(C_{\vec{x}_{1} \oplus\left(a_{1}\right)}, \ldots, C_{\vec{x}_{1} \oplus\left(a_{r}\right)}\right), \text { if } C_{\vec{x}_{1} \oplus\left(a_{r}\right)} \cap C_{\vec{x}_{2}} \neq \emptyset \\
\left(C_{\vec{x}_{1} \oplus\left(a_{r}\right)} \ldots, C_{\vec{x}_{1} \oplus\left(a_{1}\right)}\right) \text { otherwise }
\end{array}\right.
$$

and define inductively

$$
\vec{I}_{k+1}=\left\{\begin{array}{l}
\vec{I}_{k} \oplus\left(C_{\vec{x}_{k+1} \oplus\left(a_{1}\right)}, \ldots, C_{\vec{x}_{k+1} \oplus\left(a_{r}\right)}\right), \text { if } C_{\vec{x}_{k+1} \oplus\left(a_{1}\right)} \cap C_{\vec{x}_{k}} \neq \emptyset \\
\vec{I}_{k} \oplus\left(C_{\vec{x}_{k+1} \oplus\left(a_{r}\right)}, \ldots, C_{\vec{x}_{k+1} \oplus\left(a_{1}\right)}\right) \text { otherwise }
\end{array}\right.
$$

We say $\vec{B}$ reduces the $(n+1)$-chain $\overrightarrow{I_{m}}$, and write $\overrightarrow{I_{m}}=\operatorname{red}(\vec{B})$.

We will make use of a sequence of chains, which will be defined by:

- $\vec{C}(1)=\vec{C}_{S}$

- $\vec{C}(n+1)=\operatorname{red}(\vec{C}(n))$.

These have the useful property that

Lemma 5.4.7 $\sum_{C \in \vec{C}(n)}(\mu(C))^{\Delta(1)}=1, \forall n \in \mathbb{N}$. 
Proof: Since ind o red is the identity, we have $i n d(\vec{C}(n+1))=\vec{C}(n)$. Assume $\sum_{C \in \vec{C}(n)}(\mu(C))^{\Delta(1)}=1$. Then

$$
\begin{aligned}
\sum_{C \in \vec{C}(n+1)}(\mu(C))^{\Delta(1)} & =\sum_{C^{\prime} \in \text { ind }(\vec{C}(n+1))} \sum_{\substack{C \in C^{\prime} \\
C \in \widetilde{C}^{\prime}(n+1)}}(\mu(C))^{\Delta(1)} \\
& =\sum_{C^{\prime} \in \vec{C}(n)}\left(\mu\left(C^{\prime}\right)\right)^{\Delta(1)} \sum_{\substack{C \in C^{\prime} \\
C \in \mathbb{C}^{\prime}(n+1)}}\left(\frac{\mu(C)}{\mu\left(C^{\prime}\right)}\right)^{\Delta(1)} \\
& =\sum_{C^{\prime} \in \vec{C}(n)}\left(\mu\left(C^{\prime}\right)\right)^{\Delta(1)} \sum_{C \in \overrightarrow{C_{S}}}(\mu(C))^{\Delta(1)} \\
& =\sum_{C^{\prime} \in \vec{C}(n)}\left(\mu\left(C^{\prime}\right)\right)^{\Delta(1)}=1
\end{aligned}
$$

The lemma is true by (ordinary) induction.

Lemma 5.4.8 $\Delta(n)$ is the same for all $n \in \mathbb{N}$

Proof: We will proceed show that all of them are equal to $\Delta(1)$, by assuming $\Delta(n)=$ $\Delta(1)$, and show that this implies that $\Delta(n+1)=\Delta(1)$.

By the lemma above, we get that $\Delta(k) \leq \Delta(1), \forall k \in N$, so that all we need to show is that $\Delta(n+1) \nless \Delta(1)$ :

So, assume $\Delta(n+1)<\Delta(1)$. Choose $\vec{C}=\left(C_{\vec{y}}, \ldots, C_{\overrightarrow{y_{l}}}\right) \in \mathcal{C}_{n+1}$ such that $\delta(\vec{C})=$ $\Delta(n+1)$. Since $\vec{C}$ is minimal, each cell will occur at most once in $\vec{C}$, and each cell in ind $(\vec{C})$ will occur at most once in ind $(\vec{C})$.

Let $\operatorname{ind}(\vec{C})=\vec{B}=\left(C_{\vec{x}}, \ldots, C_{x_{m}}\right)$. We get

$$
\sum_{\substack{C \in \bar{C} \\ C \subset C_{\vec{x}}}}\left(\frac{\mu(C)}{\mu\left(\overrightarrow{C_{\overrightarrow{x_{i}}}}\right)}\right)^{\Delta(n+1)}=\sum_{\substack{C \in \bar{C} \\ C \subset C_{\vec{x}}}}\left(\mu\left(f_{\overrightarrow{x_{i}}}^{-1}(C)\right)\right)^{\Delta(n+1)}>\sum_{\substack{C \in \mathcal{C} \\ C \subset \subset C_{\overrightarrow{x_{i}}}}}\left(\mu\left(f_{\overrightarrow{x_{i}}}^{-1}(C)\right)\right)^{\Delta(1)}
$$

which, since $\bigoplus_{\substack{C \in \mathbb{C} \\ C \subset C_{x_{i}}}}(C) \in \mathcal{C}_{1}$, is

$$
\geq \sum_{C \in \overrightarrow{C_{S}}}(\mu(C))^{\Delta(1)}=1
$$

From this it follows

$$
1=\sum_{C \in \vec{C}}(\mu(C))^{\Delta(n+1)}
$$




$$
\begin{aligned}
& =\sum_{C^{\prime} \in i n d(\vec{C})} \sum_{\substack{C \subset C^{\prime} \\
C \in \vec{C}}}(\mu(C))^{\Delta(n+1)} \\
& =\sum_{C^{\prime} \in i n d(\vec{C})}\left(\mu\left(C^{\prime}\right)^{\Delta(n+1)} \sum_{\substack{C \subset C^{\prime} \\
C \in \vec{C}}}\left(\frac{\mu(C)}{\left.\mu\left(C^{\prime}\right)\right)}\right)^{\Delta(n+1)}\right. \\
& >\sum_{C^{\prime} \in i n d(\vec{C})}\left(\mu\left(C^{\prime}\right)\right)^{\Delta(n+1)} \\
& >\sum_{C^{\prime} \in i n d(\vec{C})}(\mu(C))^{\Delta(1)} \\
& \geq \sum_{C \in \vec{C}(n)}(\mu(C))^{\Delta(1)}=1
\end{aligned}
$$

a contradiction. So we must have $\Delta(n+1)=\Delta(1)$.

This common value will be called just $\Delta$. As the reader will notice, the fractal dimension from section $1.2, \mathrm{D}$, relates to $\Delta$ by that $D \cdot \Delta=1$.

Lemma $5.4 .9 \delta \geq 1$

Proof: Trivial from the construction.

\subsection{The metric space $(K, d)$}

Definition 12 If $x, y \in \mathbf{K}$, let $d_{n}(x, y)=\min \left\{\sum_{C \in \vec{C}}(\mu(C))^{\Delta}: \vec{C}=\left(C^{1}, \ldots, C^{k}\right)\right.$ is an $n$-chain such that $\left.x \in C^{1}, y \in C^{k}\right\}$.

Lemma 5.5.1 The sequence $\left\{d_{n}(x, y)\right\}_{n \in \mathbb{N}}$ is Cauchy for all $x, y \in \mathbb{K}$

Proof: Regard $\left\|d_{n}(x, y)-d_{n+1}(x, y)\right\|$. Let len $(\vec{C})=\sum_{C \in \vec{C}}(\mu(C))^{\Delta}$. From now on we will denote a chain as long or short according to its len-value. Forget the definition of $\vec{C}(n)$ that has been used, and let $\vec{C}(n)=\left(C^{1}, \ldots, C^{k}\right)$ and $\vec{C}(n+1)$ be chains such that $d_{i}(x, y)=\operatorname{len}(\vec{C}(i)), i=n, n+1$.

We have

$$
\operatorname{len}(\vec{C}(n)) \leq \operatorname{len}(\operatorname{ind}(\vec{C}(n+1))) \leq \operatorname{len}(\vec{C}(n))+\left(\mu\left(\vec{C}^{1}\right)\right)^{\Delta}+\left(\mu\left(\vec{C}^{k}\right)\right)^{\Delta}
$$

and

$$
\begin{aligned}
& \operatorname{len}(\vec{C}(n))-\left(\mu\left(C^{1}\right)\right)^{\Delta}-\left(\mu\left(C^{k}\right)\right)^{\Delta} \leq \operatorname{len}(\vec{C}(n+1)) \\
& \leq \operatorname{len}(\operatorname{ind}(\vec{C}(n+1)))+\sum_{i \in A}\left(\mu\left(f_{i}\left(C^{1}\right)\right)\right)^{\Delta}+\sum_{i \in A}\left(\mu\left(f_{i}\left(C^{k}\right)\right)\right)^{\Delta}
\end{aligned}
$$


so that

$$
\begin{aligned}
& \left.-\left(\mu\left(C^{1}\right)\right)\right)^{\Delta}-\left(\mu\left(f_{i}\left(C^{k}\right)\right)\right)^{\Delta}-\sum_{i \in A}\left(\mu\left(f_{i}\left(C^{1}\right)\right)\right)^{\Delta}-\sum_{i \in A}\left(\mu\left(f_{i}\left(C^{k}\right)\right)\right)^{\Delta} \\
& \left.\leq \operatorname{len}(\vec{C}(n))-\operatorname{len}(\vec{C}(n+1))) \leq\left(\mu\left(C^{1}\right)\right)\right)^{\Delta}+\left(\mu\left(f_{i}\left(C^{k}\right)\right)\right)^{\Delta}
\end{aligned}
$$

Then

$$
\|\operatorname{len}(\vec{C}(n))-\operatorname{len}(\vec{C}(n+1))\| \leq\left(\mu\left(C^{1}\right)\right)^{\Delta}+\left(\mu\left(C^{k}\right)\right)^{\Delta}+\sum_{i \in A}\left(\mu\left(f_{i}\left(C^{1}\right)\right)\right)^{\Delta}+\sum_{i \in A}\left(\mu\left(f_{i}\left(C^{k}\right)\right)\right)^{\Delta}
$$

whereupon $\left\|d_{n}(x, y)-d_{n+1}(x, y)\right\| \leq\left\|2 \cdot\left(\mu\left(C^{\max _{n}}\right)\right)^{\Delta}+2 \cdot|A| \cdot\left(\mu\left(C^{\max _{n+1}}\right)\right)^{\Delta}\right\|$, where $C^{\text {max }_{k}}$ is the $k$-cell with the largest measure.

$$
\begin{array}{r}
\left\|d_{n}(x, y)-d_{n+1}(x, y)\right\| \leq 2 \cdot\left(\left(\left(\mu\left(C^{\max _{1}}\right)\right)^{n}\right)^{\Delta}+|A| \cdot\left(\left(\mu\left(C^{\max _{1}}\right)\right)^{n+1}\right)^{\Delta}\right) \\
=2 \cdot\left(\mu\left(C^{\max _{1}}\right)\right)^{n \cdot \Delta} \cdot\left(1+|A| \cdot\left(\mu\left(C^{\max 1}\right)\right)^{\Delta}\right)
\end{array}
$$

So if $m, n \geq N, m<n$, we have

$$
\begin{aligned}
\left\|d_{m}(x, y)-d_{n}(x, y)\right\| & \leq \sum_{i=m}^{n-1}\left\|d_{i+1}(x, y)-d_{i}(x, y)\right\| \\
& \leq \sum_{i=N}^{\infty}\left\|d_{i+1}(x, y)-d_{i}(x, y)\right\| \\
& =\sum_{i=N}^{\infty}\left(\left(\mu\left(C^{\max _{1}}\right)\right)^{\Delta}\right)^{i} \cdot\left(2+2 \cdot|A| \cdot\left(\mu\left(C^{\text {max }_{1}}\right)\right)^{\Delta}\right) \\
& =\left(2+2 \cdot|A| \cdot\left(\mu\left(C^{\max _{1}}\right)\right)^{\Delta}\right) \cdot \frac{1}{1-\left(\mu\left(C^{\max _{1}}\right)\right)^{\Delta}} \cdot\left(\mu\left(C^{\text {max }_{1}}\right)\right)^{\Delta \cdot N} \\
& =\varepsilon(N)
\end{aligned}
$$

If we then let $r=\left(\mu\left(C^{m a x_{1}}\right)\right)^{\Delta}$, we see that $\varepsilon(N)=\varepsilon(0) \cdot r^{N}$, with $r<1$

We are then able to define $d(x, y)=\lim _{n \rightarrow \infty} d_{n}(x, y)$

Theorem 5.5.2 The function $d(\cdot, \cdot): K \times \mathbf{K} \rightarrow \mathbf{R}_{0}^{+}$is a metric.

Proof: (ia) $d(x, x)=\lim _{n \rightarrow \infty} d_{n}(x, x) \leq \lim _{n \rightarrow \infty}\left(\mu\left(C^{\max _{1}}\right)\right)^{n \cdot \Delta}=0$

(ib) If $x \neq y$, then $x$ and $y$ can, by proposition 5.3.14, be separated by disjoint neighbourhoods, which means that we can find an $n \in \mathrm{N}$ such that the shortest $n$-chain has to consist of at least $3 n$-cells, and accordingly, for each $m>n$ the $n$-chain $(m-n)$ induced by the shortest $m$-chain has to consist of at least 3 elements as well. Let $\vec{B}$ be 
the $m$-chain, and let $\vec{C}=\left(C^{1}, C^{2}, C^{3}\right)$ be the induced $n$-chain, and let $\vec{B}_{i}, i=1,2,3$ be such that $\vec{B}=\vec{B}_{1} \oplus \vec{B}_{2} \oplus \vec{B}_{3}$, and $\vec{B}_{i}(m-n)$-induces $\left(C^{i}\right)$. It is then easy to see that $\sum_{B \in \vec{B}_{2}}(\mu(B))^{\Delta}=\left(\mu\left(C^{2}\right)\right)^{\Delta}$, such that $d_{m}(x, y) \geq\left(\mu\left(C^{2}\right)^{\Delta} \geq\left(\mu\left(C^{\min _{n}}\right)\right)^{\Delta}\right.$ for all $m>n$, such that $d(x, y) \geq\left(\mu\left(C^{\min _{n}}\right)\right)^{\Delta}>0$

(ii) Since $d_{n}(x, y)=d_{n}(y, x)$, for all $n \in N, d(x, y)=d(y, x)$ follows, in the limit.

(iii) Given three points $x, y, z \in \mathbf{K}$, we see that for each $n \in \mathbb{N}$ the length of the composed chain from $x$ to $z$ we get by putting together a chain from $x$ to $y$ and a chain from $y$ to $z$, is less than or equal to the sum of the lengths of the two component chains, so that $d_{n}(x, y)+d_{n}(y, z) \geq d_{n}(x, z)$, and thereby $d(x, y)+d(y, z) \geq d(x, z)$.

Lemma 5.5.3 The diameter of $\mathbf{K}$ is finite.

Proof: $\sup \left\{d_{1}(x, y): x, y \in \mathbf{K}\right\} \leq \sum_{i \in A}\left(\mu\left(C_{i}\right)\right)^{\Delta}$. But for every pair $x, y \in \mathbf{K}$, we have $\left\|d_{1}(x, y)-d(x, y)\right\| \leq \varepsilon(0)$, so that $\operatorname{diam}(\mathbf{K})=\sup \{d(x, y): x, y \in \mathbf{K}\} \leq \sum_{i \in A}\left(\mu\left(C_{i}\right)\right)^{\Delta}+$ $\varepsilon(0)$, which is finite.

Lemma 5.5.4 The $f_{i}$ are contractions on $\mathbf{K}$ with factors of contractivity $r_{i}=\left(\mu_{1}(i)\right)^{\Delta}$, and for each $f_{i}$ there is an $\varepsilon_{i}>0$ such that if $d(x, y) \leq \varepsilon_{i}$, then $d\left(f_{i}(x), f_{i}(y)\right)=r_{i} \cdot d(x, y)$.

\section{Proof:}

$$
\begin{aligned}
d_{n}\left(f_{i}(x), f_{i}(y)\right) & =\min \left\{\sum_{C \in \vec{C}}(\mu(C))^{\Delta}: \vec{C} \text { is an n-chain from } f(x) \text { to } f(y)\right\} \\
& \leq \min \left\{\sum_{C \in \vec{C}}\left(\mu\left(f_{i}(C)\right)\right)^{\Delta}: \vec{C} \text { is an }(n-1) \text {-chain from } x \text { to } y\right\} \\
& =\left(\mu_{1}(i)\right)^{\Delta} \cdot \min \left\{\sum_{C \in \vec{C}}\left(\mu\left(f_{i}(C)\right)\right)^{\Delta}: \vec{C} \text { is an }(n-1) \text {-chain from } x \text { to } y\right\}
\end{aligned}
$$

so that $d\left(f_{i}(x), f_{i}(y)\right) \leq r_{i} \cdot d(x, y)$, where $r_{i}=(\mu(i))^{\Delta}$.

If $d\left(f_{i}(x), f_{i}(y)\right)<r_{i} \cdot d(x, y)$, choose a natural number $N$ so large that if $n \geq N$, then $d_{n}\left(f_{i}(x), f_{i}(y)\right)<r_{i} \cdot d_{n}(x, y)$

This means there is an $n$-chain $\vec{C}$ between $f_{i}(x)$ and $f_{i}(y)$ with len $(\vec{C})=d_{n}\left(f_{i}(x), f_{i}(y)\right)$ such that $\operatorname{len}(\vec{C})<(\mu(i))^{\Delta} \cdot d_{n-1}(x, y)$. It can then not be that $\bigcup_{C \in \vec{C}} C \subset C_{i}$, as this would make $f_{i}^{-1}(\vec{C})$ an $(n-1)$-chain from $x$ to $y$, with $\operatorname{len}\left(f_{i}^{-1}(\vec{C})\right)<d_{n-1}(x, y)$, which is a contradiction. But that means $f_{i}\left(F^{(0)}\right) \subset \bigcup_{C \in \vec{C}} C$, which again means there is an $n$-chain $\vec{B}$ between the elements of $f_{i}\left(F^{(0)}\right)$ with $\vec{B} \triangleleft \vec{C}$. That implies $d_{n}(f(x), f(y)) \geq$ $d_{n}\left(f_{i}(0), f_{i}(1)\right)$, when $n \geq N$, so that $r_{i} \cdot d(x, y)>d\left(f_{i}(x), f_{i}(y)\right) \geq d\left(f_{i}(0), f_{i}(1)\right)$, or if we 
divide by $r_{i}, d(x, y)>\frac{d\left(f_{i}(0), f_{i}(1)\right)}{r_{i}}=\varepsilon_{i}$. So if $d(x, y) \leq \varepsilon_{i}$, then $d\left(f_{i}(x), f_{i}(y)\right)=r_{i} \cdot d(x, y) \square$

Corollary 5.5.5 The functions $f_{\vec{a}}$ are contractions on $\mathbf{K}$ with factors of contractivity $r_{\vec{a}}=\left(\mu_{\mathbb{N}}([\vec{a}])\right)$, and for each $f_{\vec{a}}$ there is an $\varepsilon_{\vec{a}}>0$ such that if $d(x, y) \leq \varepsilon_{\vec{a}}$, then $d\left(f_{\vec{a}}(x), f_{\vec{a}}(y)\right)=r_{\vec{a}} \cdot d(x, y)$.

Proposition 5.5.6 The topology given by the metric $d$ is equivalent to $\Theta$

Proof: Let $B_{\varepsilon}(x)=\{y \in \mathbf{K}: d(x, y)<\varepsilon\}$. Then $\left\{B_{\varepsilon}(x)\right\}_{\varepsilon>0}$ is a neigbourhood basis for $x$ in the topology given by the metric $d$. Given an $\varepsilon>0$ there is an $n \in N$ such that $\operatorname{diam}(A) \cdot\left(\left(\mu\left(C^{\max }\right)\right)^{\Delta}\right)^{n}<\varepsilon$. We will then have, for each $x \in \mathbf{K}$ that $\sup \left\{d(x, y): y \in U^{n}(x)\right\}<\varepsilon$. Thus there is an $U^{n}(x) \subset B_{\varepsilon}(x)$ for each $B_{\varepsilon}(x)$. Take $U^{n}(x)$; if $x \in F^{(n)}$, then $d\left(x, \mathbf{K}-U^{n}(x)\right)=\min \left\{l e n(C): C\right.$ is an $n$-cell, $\left.C \subset U^{n}(x)\right\}=$ $\eta>0$. That means $B_{\eta}(x) \cap\left(\mathbf{K}-U^{n}(x)\right)=\emptyset$, so that $B_{\eta}(x) \subset U^{n}(x)$.

Corollary 5.5.7 $(\mathrm{K}, d)$ is a complete metric space.

Corollary 5.5.8 $(\mathrm{K}, d)$ er totally bounded.

Proof: Compact metric spaces are complete and totally bounded.

Let $U^{k}(x)$ and $U^{k}(y)$ be two disjoint neighbourhoods of $x$ and $y$, respectively. The boundary of $U^{n}(x), \partial U^{n}(x)$ is a finite set. If we let $\vec{C}(m)$ be a shortest $m$-chain from $x$ to $y$, the union of its elements have to intersect $\partial U^{n}(x)$ non-emptily, and we get that $\bigcap_{i \in N} \bigcup_{m=i}^{\infty} \cup_{C \in \widetilde{C}(m)} C$ has to contain at least one element of $\partial U^{n}(x)$. Pick one such, $z$, and let $\mathbf{N}_{z}=\{n \in \mathbb{N}: n \geq k, \exists C \in \vec{C}(n)$ such that $z \in C\}$. If $n \in \mathbb{N}_{z}$, then $d_{n}(x, y)=d_{n}(x, z)+d_{n}(z, y)$. If the limit of a sequence $\left\{a_{n}\right\}_{n \in \mathrm{N}}$ exists, it is equal to the limit of a subsequence $\left\{a_{n_{k}}\right\}_{k \in \mathrm{N}}$, so that, in our case

$$
d(x, y)=\lim _{k \rightarrow \infty}\left(d_{n_{k}}(x, y)\right)=\lim _{k \rightarrow \infty}\left(d_{n_{k}}(x, z)\right)+\lim _{k \rightarrow \infty}\left(d_{n_{k}}(z, y)\right)=d(x, z)+d(z, y)
$$

We have shown

Lemma 5.5.9 Let $x \neq y, x, y \in \mathbf{K}$. Then there is a $z \in \mathbf{K}, z \neq x, z \neq y$ such that $d(x, y)=d(x, z)+d(z, y)$

This is often referred to as the 4. metric Axiom. It is true for the Euclidian metric on $\mathbf{R}^{n}$, but generally not true for the subspaces of $\mathbf{R}^{n}$ with the inherited metric. If a space satisfies this Axiom, and is also complete with regards to its metric, a stronger statement is true; between two points $x$ and $y$ in the space there is a curve isometrically isomorphic to $[0, d(x, y)]$. This is what I will now set out to prove. 
Definition 13 We call $M$ a metric middle set for $x$ and $y$ if

1. $d(x, y)=d(x, m)+d(m, y), \forall m \in M$

2. If $m^{\prime} \neq m$, and $m, m^{\prime} \in M$, Then either

(a) $d(x, m)=d\left(x, m^{\prime}\right)+d\left(m^{\prime}, m\right)$, or

(b) $d(y, m)=d\left(y, m^{\prime}\right)+d\left(m^{\prime}, m\right)$

We will find a maximal such set $\Gamma$, i.e. a set such that there is no $z \in \mathbb{K}, z \notin \Gamma$, such that $\Gamma \cup\{z\}$ satisfy the criterions above.

Existence: Let $Z$ be the set of metric middle sets, ordered by inclusion. If $\kappa$ is a linearly ordered subset of $Z$, then $\Gamma^{\prime}=\bigcup_{M \in Z} M$ is an upper limit. $\Gamma^{\prime}$ is clearly a metric middle set, and by $5.5 .9, Z$ is non-empty. By Zorn's lemma we get our maximal metric middle set, $\Gamma$.

Then $\Gamma$ is:

Closed: Let $\left\{z_{n}\right\}_{n \in \mathbb{N}}$ be a sequence from $\Gamma$, such that $\lim _{n \rightarrow \infty} z_{n}=z$ exists. Then

1. $d(x, y)=\lim _{n \rightarrow \infty}(d(x, y))=\lim _{n \rightarrow \infty}\left(d\left(x, z_{n}\right)+d\left(z_{n}, y\right)\right)=\lim _{n \rightarrow \infty}\left(d\left(x, z_{n}\right)\right)+$ $\lim _{n \rightarrow \infty}\left(d\left(z_{n}, y\right)\right)=d(x, z)+d(z, y)$

2. Fix $t \in \Gamma$. For all $z_{n}$, either

(a) $d\left(x, z_{n}\right)=d(x, t)+d\left(t, z_{n}\right)$, or

(b) $d\left(y, z_{n}\right)=d(y, t)+d\left(t, z_{n}\right)$ holds

One of these has to be true for infinitely many $z_{n}$, and by going to the limit, this is true for $z$ too. We got to have $z \in \Gamma$, for if not, $\Gamma \cup\{z\}$ is a metric middle set, contrary to $\Gamma$ 's maximality.

Connected: Assume $\Gamma$ is not connected. Let $S_{\lambda}, \lambda \in \Lambda$, be the connected components. By the closedness of $\Gamma$ they have to be closed, and thereby compact. Let $a, b$ be such that $d\left(S_{a}, S_{b}\right)=\max _{\lambda \in \Lambda}\left\{\min _{\zeta \in \Lambda, \zeta \neq \lambda}\left\{d\left(S_{\lambda}, S_{\zeta}\right)\right\}\right\}$. By the compactness of $S_{a}$ 's and $S_{b}$ we can find $z_{a} \in S_{a}$ and $z_{b} \in S_{b}$ such that $d\left(z_{a}, z_{b}\right)=d\left(S_{a}, S_{b}\right)$. But by lemma 5.5 .9 there is a $z \in \mathbf{K}, z \neq z_{a}, z \neq z_{b}$ such that $d\left(z_{a}, z_{b}\right)=d\left(z_{a}, z\right)+d\left(z, z_{b}\right)$. Then

1. $d(x, y)=d\left(x, z_{a}\right)+d\left(z_{a}, z_{b}\right)+d\left(z_{b}, y\right)=d\left(x, z_{a}\right)+d\left(z_{a}, z\right)+d\left(z, z_{b}\right)+d\left(z_{b}, y\right) \geq$ $d(x, z)+d(z, y) \geq d(x, y)$, so that $d(x, y)=d(x, z)+d(z, y)$

2. Given $t \in \Gamma$, either: 
(a) $d\left(x, z_{a}\right)=d(x, t)+d\left(t, z_{a}\right)$, whereupon

$$
\begin{aligned}
& d(x, z)=d\left(x, z_{a}\right)+d\left(z_{a}, z\right) \\
& =d(x, t)+d\left(t, z_{a}\right)+d\left(z_{a}, z\right) \\
& \geq d(x, t)+d\left(t, z_{a}\right) \geq d(x, z)
\end{aligned}
$$

so that $d(x, z)=d(x, t)+d(t, z)$

or

(b) $d\left(z_{a}, y\right)=d\left(z_{a}, t\right)+d(t, y)$

But then

$$
\begin{aligned}
& d\left(z_{a}, y\right)=d\left(z_{a}, z_{b}\right)+d\left(z_{b}, t\right)+d(t, y) \\
& =d\left(z_{a}, z\right)+d\left(z, z_{b}\right)+d\left(z_{b}, t\right)+d(t, y) \\
& \geq d\left(z_{a}, z\right)+d(z, t)+d(t, y) \\
& \geq d\left(z_{a}, z\right)+d(z, y)=d\left(z_{a}, y\right)
\end{aligned}
$$

so that $d(y, z)=d(y, t)+d(t, z)$

But $z \notin \Gamma$, by the definition of $S_{a}$ and $S_{b}$. We have thereby shown that $\Gamma \cup\{z\}$ is a metric middle set. But this contradicts the maximality of $\Gamma$, so we must conclude that $\Gamma$ has to be connected.

To each element $z \in \Gamma$ we can then assign a number: $\phi(z)=d(x, z) . \phi$ is an isometry, since $\left|\phi(z)-\phi\left(z^{\prime}\right)\right|=\left|d(x, z)-d\left(x, z^{\prime}\right)\right|=d\left(z, z^{\prime}\right)$. And to each real number $r \in[0, d(x, y)]$ we can find an element $z \in \Gamma$ such that $\phi(z)=d(x, z)=r$, so $\phi$ is surjective.

We have shown

Proposition 5.5.10 Given $x, y \in \mathbb{K}, x \neq y$, there is a subset of $\mathbf{K}$ containing $x$ and $y$ that is isometrically isomorphic to the interval $[0, d(x, y)]$, and in particular there is a subset of $\mathbf{K}$ that contains $F^{(0)}$ and is isometrically isomorphous to $[0,1]$

\subsection{Measures of length}

Definition $14 A$ curve in $\mathbf{K}$ is the image of an interval in $\mathbf{R}$ by a continuous mapping $\mathbf{R} \rightarrow \mathbf{K}$. Thus a curve is closed and connected.

Definition $15\left\{U_{i}\right\}_{n \in \mathbb{N}}$ is a $\delta$-cover of a set $F$ if $\operatorname{diam}\left(U_{i}\right) \leq \delta$ for all $U_{i}$, and $F \subset \bigcup_{n \in \mathbb{N}} U_{i}$. Let $\mathcal{H}_{\delta}^{1}(F)=\inf \left\{\sum_{n \in \mathbb{N}} \operatorname{diam}\left(U_{i}\right):\left\{U_{i}\right\}_{n \in \mathbb{N}}\right.$ is a $\delta$-cover of $\left.F\right\}$, and let $\ell(F)=\lim _{\delta \rightarrow 0^{+}} \mathcal{H}_{\delta}^{1}(F)=\sup _{\delta>0} \mathcal{H}_{\delta}^{1}(F)$. $\ell$ will then be 1-dimensional Hausdorffmeasure. 
Lemma 5.6.1 If $L \subset \mathbf{K}$ is a closed, connected set, and $x, y \in L$, then $\ell(L) \geq d(x, y)$, with equality if $L$ is a maximal metric middle set of $x$ and $y$.

Proof: We see that $\ell$ is the same if we use just open covers $\left\{U_{i}\right\}$ in the definition. But as $L$ is closed, $L$ is compact, so that any open cover can be reduced to a finite open cover. We have that if $A \cap B \neq \emptyset$, then $\operatorname{diam}(A \cup B) \leq \operatorname{diam}(A)+\operatorname{diam}(B)$, such that $\operatorname{diam}(L) \leq \operatorname{diam}\left(\cup_{i=1}^{n} V_{i}\right) \leq \sum_{i=1}^{n} \operatorname{diam}\left(V_{i}\right)$, where $\left\{V_{i}\right\}$ is a finite cover of $L$, so that $\ell(L) \geq d(x, y)$.

If $L$ is a metric middle set of $x$ and $y$, then $\ell(L)=d(x, y)$, as we for any $n \in \mathbb{N}$ can cover $L$ with $n$ closed balls with diameter $\frac{d(x, y)}{n}$.

Lemma 5.6.2 $\ell \circ f_{i}=r_{i} \cdot \ell$, where $r_{i}$ is the contractivity factor of $f_{i}$.

Proof: If $S$ is a set in $\mathbf{K}$, then $\operatorname{diam}(S) \geq \operatorname{diam}\left(S \cap C_{\boldsymbol{i}}\right)$, whereupon, for a set $\left\{U_{j}\right\}$, we have $\sum \operatorname{diam}\left(U_{j}\right) \geq \sum \operatorname{diam}\left(U_{j} \cap C_{i}\right)$, so that if $F$ is a subset of $C_{i}$, then $\mathcal{H}_{\delta}^{1}(F)=\left\{\sum \operatorname{diam}\left(U_{i}\right):\left\{U_{i}\right\}\right.$ is a $\delta$-cover of $F$ with $\left.U_{i} \subset C_{i}\right\}$

So if $\delta \leq \varepsilon$, and $\operatorname{diam}(S) \leq \delta$, we have $\operatorname{diam}\left(f_{i}(S)\right)=r_{i} \cdot \operatorname{diam}(S)$, such that $\mathcal{H}_{\frac{\delta}{r_{i}}}^{1}\left(f_{i}(S)\right)=$ $r_{i} \cdot \mathcal{H}_{\delta}^{1}(S)$.

Corollary 5.6.3 The shortest curve contained in $C_{\vec{a}}$ between two points $f_{\vec{a}}(x)$ and $f_{\vec{a}}(y)$ has length $r_{\vec{a}} \cdot d(x, y)$

Corollary 5.6.4 The shortest curve contained in $C_{\vec{a}}$ between $f_{\vec{a}}(\underline{0})$ and $f_{\vec{a}}(\underline{1})$ has length $r_{\vec{a}}$.

We will say $C_{\vec{a}}$ has length $r_{\vec{a}}$. We write: $\lambda\left(C_{\vec{a}}\right)=r_{\vec{a}}$.

\subsection{Symmetry}

We have this far not concerned ourselves with symmetry, as the basic topological properties of Simple Fractal spaces are independent of it. It is, though, essential in the present construction of Brownian motion. What we will prove, is

Axiom 5': There is a symmetry $U^{\prime}$ : i.e. $U^{\prime}: \mathbf{K} \rightarrow \mathbf{K}$ is an isometry such that $U^{\prime^{2}}$ is the identity, $U^{\prime}(\underline{0})=\underline{1}$ and $n$-cells are mapped onto $n$-cells for all $n \in \mathbb{N}$.

I will proceed to show that this Axiom is true. 
Definition 16 Let $U^{(0)}: F^{(0)} \rightarrow F^{(0)}$ be the mapping switching the two elements in $F^{(0)}$. Then define the mappings $U^{(k)}: F^{(k)} \rightarrow F^{(k)}$ inductively by

$$
U^{(k+1)}(x)=f_{U_{1}(i)} \circ V_{i}^{(k)} \circ f_{i}^{-1}(x) \text { for an } i \text { such that } x \in C_{i}
$$

where

$$
V_{i}^{(k)}= \begin{cases}i d & \text { if }\left\{j \in A: f_{U(i)}(\underline{1}) \in C_{U(j)}\right\} \neq\left\{j \in A: f_{i}(\underline{1}) \in C_{j}\right\} \\ U^{(k)} & \text { if }\left\{j \in A: f_{U(i)}(\underline{1}) \in C_{U(j)}\right\}=\left\{j \in A: f_{i}(\underline{1}) \in C_{j}\right\}\end{cases}
$$

Lemma 5.7.1 $U^{(n)}$ is well defined and $\left.U^{(n)}\right|_{F^{(n-1)}}=U^{(n-1)},\left(U^{(n)}\right)^{2}=i d, \forall n \in \mathbb{N}$

Proof: This proof will be an inducive one. But it will not be simple induction on one property, but a nested induction where the truth of each one of the properties at step $(n)$ is needed both for the proof of the other, and for the proof of its continuation at the step $(n+1)$. We will first show that if $U^{(n-1)}$ is well defined and switch $\underline{0}$ and $\underline{1}$, then $U^{(n)}$ is well defined:

We need only check elements belonging to more than one 1-cell. Let $x$ be one such element:

Pick $i, j \in A, i \neq j$ such that $x \in C_{i} \cap C_{j}$. If $\left|C_{i} \cap C_{j}\right|=1$, then, by the definition of $V_{i}^{(n-1)}$, we have $f_{U(i)} \circ V_{i}^{(n-1)} \circ f_{i}^{-1}(x) \in C_{U(j)}$, and symmetrically that $f_{U(j)} \circ V_{j}^{(n-1)} \circ$ $f_{j}^{-1}(x) \in C_{U(i)}$. As, by Axiom $5,\left(\left|C_{i} \cap C_{j}\right|=1\right) \Rightarrow\left(\left|C_{U(i)} \cap C_{U(j)}\right|=1\right)$, we have $f_{U(i)} \circ V_{i}^{(n-1)} \circ f_{i}^{-1}(x)=f_{U(j)} \circ V_{j}^{(n-1)} \circ f_{j}^{-1}(x)$

If $\left|C_{i} \cap C_{j}\right|=2$ we can pick for ourselves a $k \in A$ such that $\left|C_{i} \cap C_{k}\right|=\left|C_{j} \cap C_{k}\right|=1$. Let $\{x, y\}=C_{i} \cap C_{j}$, and let $\{x\}=C_{i} \cap C_{k}=C_{j} \cap C_{k}$. Then $f_{U(i)} \circ V_{i}^{(n-1)} \circ f_{i}^{-1}(x)=f_{U(k)} \circ$ $V_{k}^{(n-1)} \circ f_{k}^{-1}(x)=f_{U(j)} \circ V_{j}^{(n-1)} \circ f_{j}^{-1}(x)$. Since $f_{U(i)} \circ V_{i}^{(n-1)} \circ f_{i}^{-1}(x) \neq f_{U(i)} \circ V_{i}^{(n-1)} \circ f_{i}^{-1}(y)$ for all $i \in A$, and $\left|C_{U(i)} \cap C_{U(i)}\right|=2$, we also get $f_{U(i)} \circ V_{i}^{(n-1)} \circ f_{i}^{-1}(y)=f_{U(j)} \circ V_{j}^{(n-1)} \circ f_{j}^{-1}(y)$

If $U^{(n+1)}$ is well defined, and $\left(U^{(n)}\right)^{2}=i d$, then $\left(U^{(n+1)}\right)^{2}=i d$ is trivial from the fact that $\left(U_{1}\right)^{2}=i d$, and that $V_{i}^{(n)}=V_{U(i)}^{(n)}$

We see that for all $i \in A$ we have $\left(V_{i}^{(n)}\right)^{2}=i d$ either for all or for none of the natural numbers $n \in \mathbf{N}$.

Assume $U^{(1)}$ is well defined. We will then show that $\left.U^{(1)}\right|_{F^{(0)}}=U^{(0)}$. It is enough to show that $U^{(1)}(\underline{0})=\underline{1}$. There are $i, j \in A$ such that $\underline{0} \in C_{i}, \underline{0} \notin C_{j}$ and $C_{i} \cap C_{j} \neq \emptyset$. That implies $\left|C_{i} \cap C_{j}\right|=1$, and thereby $\left|C_{U(i)} \cap C_{U(j)}\right|=1$. That gives us $1 \notin C_{U(j)}$, but $\underline{1} \in C_{U(i)}$. And since $\underline{0} \notin C_{j}$, we know that $f_{U(i)} \circ V_{i}^{(n-1)} \circ f_{i}^{-1}(\underline{0}) \in C_{U(i)} \cap C_{U(j)}$. But $U^{1}(\underline{0}) \in f_{U(i)}\left(F^{(0)}\right)=$ $\left\{\underline{1}, C_{U(i)} \cap C_{U(j)}\right\}$, so $U^{(1)}(\underline{0})=\underline{1}$. 
$\left.V_{i}^{(n)}\right|_{F^{(n-1)}}=V_{i}^{(n-1)}$ if $\left.U_{i}^{(n)}\right|_{F^{(n-1)}}=U_{i}^{(n-1)}$. Do therefore assume $\left.U^{(n)}\right|_{F^{(n-1)}}=U^{(n-1)}$. Then

$$
\begin{aligned}
\left.U^{(n+1)}\right|_{F^{(n)}} & =\left.f_{\left.U^{*}\right)} \circ V_{*}^{(n)} \circ f_{*}^{-1}\right|_{F^{(n)}} \\
& =\left.f_{\left.U^{*}\right)} \circ V_{*}^{(n)}\right|_{F^{(n-1)}} \circ f_{*}^{-1} \\
& =f_{\left.U^{*}\right)} \circ V_{*}^{(n-1)} \circ f_{*}^{-1}=U^{(n)}
\end{aligned}
$$

We will connect the pieces, and prove the lemma:

We have shown that $U^{(1)}$ is well defined, $\left.U^{(1)}\right|_{F^{(0)}}=U^{(0)},\left(U^{(1)}\right)^{2}=i d$, and that for $n \geq 1, U^{(n+1)}$ is well defined, $\left.U^{(n+1)}\right|_{F(n)}=U^{(n)},\left(U^{(n)}\right)^{2}=i d$ if $U^{(n)}$ is well defined, $\left.U^{(n)}\right|_{F^{(n-1)}}=U^{(n-1)},\left(U^{(n)}\right)^{2}=i d, \forall n \in \mathbb{N}$.

The lemma follows by induction.

Corollary $\left.5.7 .2 U^{(m)}\right|_{F^{(n)}}=U^{(n)}$ if $n \leq m$

Proof: Inductively with the premise $\left.U^{(n+1)}\right|_{F^{(n)}}=U^{(n)}$

Definition 17 Let $U: \mathbf{K} \rightarrow \mathbf{K}$ be defined by; if $x={ }^{\circ}(\tilde{x})$, then $U(x)={ }^{\circ}\left(U^{N}(\tilde{x})\right)$ for an arbitrary $N \in \in^{*} N-N$

Since $F^{(\infty)}$ is dense in $\mathbf{K}$, for each $x \in \mathbb{K}$ there is an $\tilde{x} \in F^{(N)}$ such that $x={ }^{\circ}(\tilde{x})$.

Lemma 5.7.3 $U$ is well defined.

Proof: We see that if $n \geq m$, and $x, y \in F^{(m)}$ are two elements of the same $m$-cell, then $U^{(n)}(x)$ and $U^{(n)}(y)$ are subsets of the same $m$-cell. If $x \simeq y, x, y \in F^{(N)}$, then we can without loss of generality assume $x$ and $y$ have to be in the same $M$-cell for an $M \in^{*} \mathrm{~N}-\mathrm{N}$. For if not, we can find a third element $z$ such that $x \simeq z$ and $z \simeq y$, and the assumption holds. On the assumtion we get that $U^{(N)}(x)$ and $U^{(N)}(y)$ are in the same $M$-cell, and $U^{(N)}(x) \simeq U^{(N)}(y)$.

Lemma 5.7.4 U maps $n$-cells onto $n$-cells.

Proof: If $x$ and $y$ are two elements in the same $n$-cell, then $U^{(N)}(x)$ and $U^{(N)}(y)$ are in the same $n$-cell. 
Lemma 5.7.5 $U$ is continuous.

Proof: With $U^{n}(x)$ as $x$ s $n$-neighbourhood, we see that $U\left(U^{n}(x)\right)=U^{n}(U(x))$. This means the basic neighbourhoods of $x$ and $U(x), U^{n}(x)$ and $U^{n}(U(x))$, are in 1-1 correspondense with each other.

Lemma 5.7.6 $U(\underline{0})=\underline{1} . U^{2}$ is the identity.

Proof: Trivial from the definition of $U$.

Lemma 5.7.7 $\mu \circ U(\cdot)=\mu(\cdot)$

Proof: We need only check for equality on the cells, by the proof of lemma 5.1.2. We have, if $\vec{a}=\left(a_{1}, \ldots, a_{n}\right)$, that $\mu\left(C_{\vec{a}}\right)=\prod_{i=1}^{n} \mu_{1}\left(k_{i}\right)$, where $k_{i}$ is either $a_{i}$ or $U_{1}\left(a_{i}\right)$. Since $\mu_{1}\left(U_{1}\left(a_{i}\right)\right)=\mu\left(a_{i}\right)$, we have $\mu\left(C_{\vec{a}}\right)=\prod_{i=1}^{n} \mu_{1}\left(a_{i}\right)=\mu\left(C_{\vec{a}}\right)$

Proposition 5.7.8 Axiom 5' is true.

Proof: We need only check the metric. This is simple, as $U$ gives a 1-1 correspondense between chains, giving $d_{n}(x, y)=d_{n}(U(x), U(y))$, and, when we go to the limit, $d(x, y)=d(U(x), U(y))$. 


\section{References}

[1] Albeverio, Sergio, Fenstad, Jens Erik, Høegh-Krohn, Raphael \& Lindstrøm, Tom: Nonstandard Methods in Stochastic Analysis and Mathematical Physics.

Academic Press, Inc. 1986. ISBN 0-12-048860-4

[2] Bandt, Cristoph: Self-similar Sets 2. Invariant Factors of One-sided Shifts. Sektion Mathematik, Ernst-Moritz-Arndt-Universität, 2200 Greifswald, Germany.

[3] Bandt, Cristoph \& Stahnke, Jörg: Self-similar Sets 6. Interior Distance on Deterministic Fractals. Sektion Mathematik, ErnstMoritz-Arndt-Universitåt, 2200 Greifswald, Germany.

[4] Barlow, Martin: Random Walks, Electrical Resistance, and Nested Fractals. To appear in K.D. Elworthy and N. Ikeda: "Asymptotic Problems in Probability Theory", Pitman Publishing Co.

[5] Barnsley, Michael: Fractals Everywhere.

Academic Press, Inc. 1988. ISBN 0-12-079062-9

[6] Breiman, Leo: Probability.

Addison-Wesley Publishing Company, Inc. 1968. Library of Congress Catalog Card No. 68-10842

[7] Cutland, Nigel (ed.): Non-standard Analysis and its Applications. Cambridge University Press. 1988. ISBN 0-52-135109-X

[8] Doyle, Peter G. \& Snell, J. Laurie: Random Walks and Electric Networks. ISBN 0-88385-024-9

[9] Falconer, Kenneth: Fractal Geometry, Mathematical Foundations and Applictions.

John Wiley \& Sons Ltd. 1990. ISBN 0471922870

[10] Hurd, A.E. and Loeb, P.A.: An Introduction to Nonstandard Real Analysis. Academic Press 1985. ISBN 0-12-362440-1 
[11] Hutchinson, John E.: Fractals and Self Similarity. Indiana University Mathematics Journal, Vol. 30, No. 5 (1981)

[12] Lindstrøm,Tom: Brownian Motion on NestedFractals. Memoirs Amer. Math. Soc. 420, 1990

[13] Parthasarathy, K.R.: Introduction to Probability and Measure. The Macmillan Press, Ltd. 1977. ISBN 0-333-21855-8

[14] Seneta, E.: Non-negative Matrices.

George Allen \& Unwin Ltd. 1973. ISBN 0-04-519011-9

[15] Wicks, Keith R.: Fractals and Hyperspaces. Springer-Verlag Berlin Heidelberg1991. ISBN 3-540-54965-X 